\title{
Exact kinematics in the small $x$ evolution of the color dipole and gluon cascade
}

\author{
Leszek Motyka \\ II Institute for Theoretical Physics, University of Hamburg, Luruper Chaussee 149, D-22761, Germany \\ Institute of Physics, Jagellonian University Reymonta 4, 30-059 Kraków, Poland \\ Anna M. Staśto \\ Physics Department, Penn State University, University Park, 16802 PA, U.S.A. \\ RIKEN center, Brookhaven National Laboratory, Upton, 11973 NY, U.S.A. \\ Institute of Nuclear Physics, Polish Academy of Science, ul. Radzikowskiego 152, Kraków, Poland
}

\begin{abstract}
The problem of kinematic effects in gluon and color dipole cascades is addressed in the large $N_{c}$ limit of $S U\left(N_{c}\right)$ Yang-Mills theory. We investigate the tree level multi-gluon components of the gluon light cone wave functions in the light cone gauge keeping the exact kinematics of the gluon emissions. We focus on the components with all helicities identical to the helicity of the incoming gluon. The recurrence relations for the gluon wave functions are derived. In the case when the virtuality of the incoming gluon is neglected the exact form of the multi-gluon wave function is obtained. Furthermore, we propose an approximate scheme to treat the kinematic effects in the color dipole evolution kernel. The new kernel entangles longitudinal and transverse degrees of freedom and leads to a reduced diffusion in the impact parameter. When evaluated in the next-to-leading logarithmic (NLL) accuracy, the kernel reproduces the correct form of the double logarithmic terms of the dipole size ratios present in the exact NLL dipole kernel. Finally, we analyze the scattering of the incoming gluon light cone components off a gluon target and the fragmentation of the scattered state into the final state. The equivalence of the resulting amplitudes and the maximally-helicityviolating amplitudes is demonstrated in the special case when the target gluon is far in rapidity from the evolved gluon wave function.
\end{abstract}

\section{INTRODUCTION}

In this paper we investigate the possibility of using information available in the exact tree level QCD amplitudes in the large $N_{c}$ limit to improve the evolution equations of QCD. Following the Mueller's color dipole model approach [1, 2] we choose the framework of the light cone perturbation theory (LCPT) [3, 4, 5, 6] and adopt the light cone gauge. The attractive feature of the color dipole model is its probabilistic interpretation in terms of the multi-dipole densities. This allows to extract the total cross-section for a scattering of two small sized onia (quark-antiquark systems) at a large energy, $\sqrt{s}$, from the tree level amplitudes in the soft-gluon limit. The color dipole model provides a resummation scheme of the leading logarithmic (LL) contributions in powers of $\alpha_{s} \log s$. It is remarkable, that the analysis of the tree level amplitudes in the color dipole framework reproduces the results of the leading logarithmic Balitsky-Fadin-Kuraev-Lipatov (BFKL) evolution equation [7], that incorporates quantum loops. This suggests that a more accurate treatment of the color dipole (or gluon) cascade at the tree level but beyond the soft gluon limit, may provide a lot of insight into higher order corrections to the BFKL equation.

The basic object in our analysis is the light cone wave function of a gluon, that may be decomposed into multi-gluon components. The whole analysis of this paper is carried out in the large $N_{c}$ limit. As the simplest starting point we choose the configuration in which both the initial state gluon and all the gluons in the wave function carry the positive helicity. We aim at obtaining an exact description of the gluon cascade, so we keep the complete information about the gluon kinematic variables along the cascade. This implies that both the form of gluon splitting vertex and the energy denominators of the light cone perturbation theory are treated exactly. This is different from the soft limit where the approximate forms were used.

As the first result, we find an exact form of a tree level multi-gluon components of the real gluon wave function in the chosen helicity sector. This is achieved by solving recurrence relations for the multi-gluon components of the gluon light cone wave function in the momentum space. The natural variables in this formulation are closely related to the spinor products which are used in the construction of the maximally-helicity-violating amplitudes (MHV), see e.g. [8, 9]. This wave function can be also analyzed in the transverse coordinate space, after a suitable Fourier transform is performed. Curiously enough, the obtained wave function in the coordinate space coincides with the one obtained 
in the soft gluon limit. This coincidence, however, is expected to hold only for the particular choice of helicities, that has been assumed.

In general, the description of the gluon cascade within LCPT is non-local, the energy denominators involve momenta of all gluons, and that makes the resummation difficult in a general case. In order to obtain a more practical improvement of the dipole model kernel, we propose a local approximation to the $1 \rightarrow 2$ gluon splitting kernel in which the value of energy denominator is obtained from the invariant mass of the produced gluon pair. This leads to a new color dipole kernel. The form of the kernel in the soft gluon limit $\frac{d z}{z} d^{2} \underline{x}_{2} \frac{x_{01}^{2}}{x_{02}^{2} x_{21}^{2}}$ gets modified to

$$
\frac{d z}{z} z \frac{d^{2} \underline{x}_{2}}{x_{01}^{2}}\left[K_{1}^{2}\left(\frac{x_{02}}{x_{01}} \sqrt{z}\right)+K_{1}^{2}\left(\frac{x_{12}}{x_{01}} \sqrt{z}\right)-2 K_{1}\left(\frac{x_{02}}{x_{01}} \sqrt{z}\right) K_{1}\left(\frac{x_{12}}{x_{01}} \sqrt{z}\right) \frac{\underline{x}_{02} \cdot \underline{x}_{12}}{x_{02} x_{12}}\right]
$$

where $\underline{x}_{01}$ is the parent dipole vector, $\underline{x}_{02}, \underline{x}_{21}$, are the daughter dipole vectors, $z$ is the fraction of the longitudinal momentum of the softer daughter gluon, and $K_{1}$ is the modified Bessel function. The modified kernel becomes equivalent to the leading logarithmic kernel for $z x_{02}^{2} \ll x_{01}^{2}$ and $z x_{21}^{2} \ll x_{01}^{2}$ but differs significantly otherwise. In more detail, the production of larger dipole sizes is exponentially suppressed above the cutoff size which depends on the longitudinal momentum fraction of the soft gluon. The modified kernel introduces corrections at all orders beyond the leading logarithmic approximation. In particular, we show that the modified kernel reproduces an important part of the NLL kernel, namely the double logarithmic terms. Interestingly enough, these are the dominant terms that violate the two dimensional conformal symmetry of the BFKL-dipole kernel both in $\mathcal{N}=4$ Supersymmetric YangMills (SYM) theory and in QCD at NLL approximation [10, 11, 12, 13, 14]. The suppression of the large dipole emission implies also a suppression of the diffusion in the impact parameter.

Finally, we consider the production amplitude of $n$ gluons in a scattering process of two gluons. We impose additional conditions that the produced state is arbitrarily far away in rapidity from the target gluon, and that both the incoming and all the outgoing gluons carry positive helicities. Using the LCPT we reproduce formally the Parke-Taylor form of the MHV amplitudes [8] in this limit. In the intermediate steps of the proof we analyze in detail the fragmentation amplitude of $m$ virtual gluons into $n \geq m$ real gluons. We find that an apparent entanglement of the fragmentation process of different gluons in LCPT can be unwound at the tree level, and in fact, fragmentation amplitude of each of the gluons is independent. We find an exact form of the tree level amplitude for a gluon fragmentation, again assuming that the helicity of the incoming and outgoing gluons are positive. We observe, that the $n$-gluon component of the incoming real gluon wave function is dual to the fragmentation amplitude of a gluon into $n$ real gluons i.e. the latter has the same form as the former under replacement of the gluon transverse positions by the gluon transverse (light cone) velocities.

The structure of this paper is as follows: in the next section we present the overview of the color dipole model and the next-to-leading order corrections to BFKL. In Sec. III we set up a general formalism for computing the multi-gluon tree-level wave function with exact kinematics in the light cone perturbation theory and in the light cone gauge. We derive the recurrence relations for the wave functions with different number of external gluons in momentum space and in transverse coordinate space. In Sec. IV we derive the modified kernel for the dipole evolution in transverse coordinate space. We show that when expanded to the next-to-leading order in the strong coupling constant the double logarithmic terms in the next-to-leading calculation are recovered. We discuss the implications for the impact parameter dependence of the cross section. In Sec. $\mathrm{V}$ we construct the scattering amplitudes for external on-shell partons and demonstrate the general factorization property of the fragmentation in the light cone perturbation theory. The equivalence with the MHV amplitudes is also demonstrated in the high energy limit. In the last section we briefly summarize our results. The Appendix contains the most technical part of the calculations.

\section{OVERVIEW OF COLOR DIPOLE EVOLUTION IN QCD}

In a conventional approach to the high energy limit one computes the amplitude for scattering of two highly energetic particles which interact via exchange of gluons in the $t$-channel. The emitted gluons in this scattering process are then resummed in the multi-Regge kinematics. The amplitude turns out to be governed by the exchange of a Reggeon with the quantum numbers equivalent to that of the vacuum: the Pomeron. Its intercept is evaluated from the solution to the Balitsky-Fadin-Kuraev-Lipatov (BFKL) 7] evolution equation at small $x$. This equation describes the change of the amplitude with increasing rapidity between the scattering initial particles. In the groundbreaking paper [1] Mueller demonstrated that the small- $x$ cascade of gluons can be alternatively described as the evolution of the hadron wave function. The subsequent emissions of the soft gluons in the hadron wave function can be computed in the light cone perturbation theory. By soft gluons we mean here gluons whose longitudinal momentum fractions are much smaller than that of the incoming particles, the transverse momenta of the gluons are not restricted. In the large $N_{c}$ limit it was demonstrated that the correct degrees of freedom at high energy are $q \bar{q}$ dipoles. Furthermore, by taking 
the Fourier transform with respect to the transverse momenta it was shown that the soft gluon emissions factorize in the transverse coordinate space. The resulting evolution equation for the scattering amplitude was then formulated by considering the interaction of the hadron wave function with the target. It can be written in the following form

$$
\frac{\partial N_{Y}\left(\underline{x}_{01}\right)}{\partial Y}=\bar{\alpha}_{s} \int \frac{d^{2} \underline{x}_{02}}{2 \pi} \frac{\underline{x}_{01}^{2}}{\underline{x}_{12}^{2} \underline{x}_{02}^{2}}\left[N_{Y}\left(\underline{x}_{0}, \underline{x}_{2}\right)+N_{Y}\left(\underline{x}_{1}, \underline{x}_{2}\right)-N_{Y}\left(\underline{x}_{0}, \underline{x}_{1}\right)\right] .
$$

Here, $N_{Y}$ is the scattering amplitude for a dipole on the target, $Y$ is the rapidity for the process and $\underline{x}_{0}, \underline{x}_{1}, \underline{x}_{2}$ are the two-dimensional vectors which specify the position of the color dipoles. To obtain the solution to this equation one needs to specify an amplitude at the initial rapidity $N_{Y_{0}}$. The solution to this equation for the dipole scattering amplitude turned out to be equivalent to the one from the original BFKL equation, at least in the case of the inclusive quantities [2], like the total cross section.

The dipole evolution proved to be a powerful framework for investigating the unitarity corrections [15, 16] in the scattering processes. By taking into account multiple rescatterings of the gluon components of the evolved hadron wave function onto the nuclei the non-linear evolution equation was derived [17, 18]. It differs from (2) by the presence of an additional nonlinear term

$$
-N_{Y}\left(\underline{x}_{0}, \underline{x}_{2}\right) N_{Y}\left(\underline{x}_{1}, \underline{x}_{2}\right),
$$

which tames the growth of the amplitude with the energy and leads to the gluon saturation. The same equation was derived in the context of the high energy operator expansion with Wilson lines [19, 20, 21, 22, 23, 24, 25] and in the Color Glass Condensate framework [26, 27, 28], see also [29] for a nice review. It is called the Balitsky-Kovchegov equation. It is important to note that the kernels both in the linear and non-linear equations are exactly the same (to the NLL level in the dipole approach).

\section{A. Next-to-leading corrections and the kinematical effects}

Similarly to the $t$-channel approach the dipole evolution kernel receives large corrections at next-to-leading order. The next-to-leading order kernel in the momentum space was first evaluated in [30, 31, 32, 33] in the forward case, and later in [34, 35] in the non-forward case. The dipole kernel at next-to-leading accuracy was then computed in [10, 36] via direct calculation and also in [11, 12, 13, 14] by performing the Fourier transform of the existing result for the non-forward BFKL kernel in the momentum space. The running coupling corrections to the dipole kernel were also evaluated in [37]. There are different sources of the next-to-leading corrections and it is quite well known that the major part of them which is common to QCD and $\mathcal{N}=4$ Super Yang Mills theory comes from the kinematics, see for example [38, 39, 40]. The slow convergence of the small $x$ series can be argued intuitively as follows. In the standard perturbative collinear approach one identifies the large scale $\mu^{2}$ which characterizes the short distance part of the process with strong interactions. The limit of large $\mu^{2}$ implies automatically the smallness of the strong coupling constant, which allows to seek the solution to the hard scattering cross section and to the splitting function in the form of the perturbative series. This series should be convergent at least in the asymptotic sense. In the high energy limit the large parameter is the total available energy $\sqrt{s}$, the strong coupling however is not naturally small in this limit, and can take large values too. Therefore there is no guarantee that the expansion in powers of the strong coupling in the high energy limit will be strongly convergent. Indeed, it is observed that the small $x$ expansion is poorly convergent, see for example [40, 41, 42]. By assuming the multi-Regge kinematics one makes strong approximations onto the phase space of the produced particles. As a result the perturbative methods which rely on the expansion in terms of the coupling are simply inefficacious in correcting these kinematic approximations at higher orders.

Even before the next-to-leading correction to the BFKL kernel was computed, it was shown that the constraints from the more careful treatment of the kinematics (while being formally of the higher order) are numerically very important and significantly reduce the growth of the gluon density [38, 39, 40]. Taking this effect into account leads to the predictions which are in a good agreement with phenomenology [43, 44, 45]. It was first demonstrated by Salam [41, 46] that the kinematic effects manifest themselves at next-to-leading order in the form of the double logarithms of ratios of transverse momenta, and correspond to the higher order poles in the Mellin space conjugated to the transverse momenta. The formalism of the collinear resummation developed later [47, 48, 49, 50, 51, 52] was shown to stabilize the small $x$ series by imposing onto the kernel the kinematic constraints and the momentum sum rule. The small $x$ expansion is also stabilized by the appropriate implementation of the running coupling effects, in the case of QCD. Another approach to match the DGLAP and BFKL resummations was constructed in parallel in Refs. [53, 54, 55, 56, 57, 58] and similar results were found. Finally, the same problem was addressed in yet another scheme in Ref. [59, 60].

It is therefore quite urgent to address the problem of the better treatment of the kinematics in the context of the dipole evolution. As the next-to-leading corrections take into account only part of the kinematic corrections it 
is necessary to investigate the origin and effect of the kinematical constraints on the dipole evolution. Such effects necessarily go beyond the fixed order calculation and are part of the all-order (in powers of $\alpha_{s}^{n} \ln ^{m} s$ ) resummation.

\section{EXACT KINEMATICS IN THE MULTI-GLUON WAVE FUNCTION}

\section{A. Setup of the problem}

In this section we shall derive the exact form of the $n$-gluon components of the real gluon wave function, $\Psi_{n}, n=$ $1,2, \ldots$, using the LCPT for a special choice of helicities. Thus, we shall assume that the incoming gluon has the positive helicity and analyze the case when all the gluons in the wave function have all positive helicities as well. Such choice of the helicity state is dictated by an expected simplicity of the result. This choice also prepares the ground for the comparison with the Parke-Taylor amplitudes that we shall make in Sec. $\nabla$. We understand the computation presented in this section as a demonstration of a potentially useful calculational technique and the first step towards the exact determination of the gluon wave function with arbitrary helicities at the tree level.

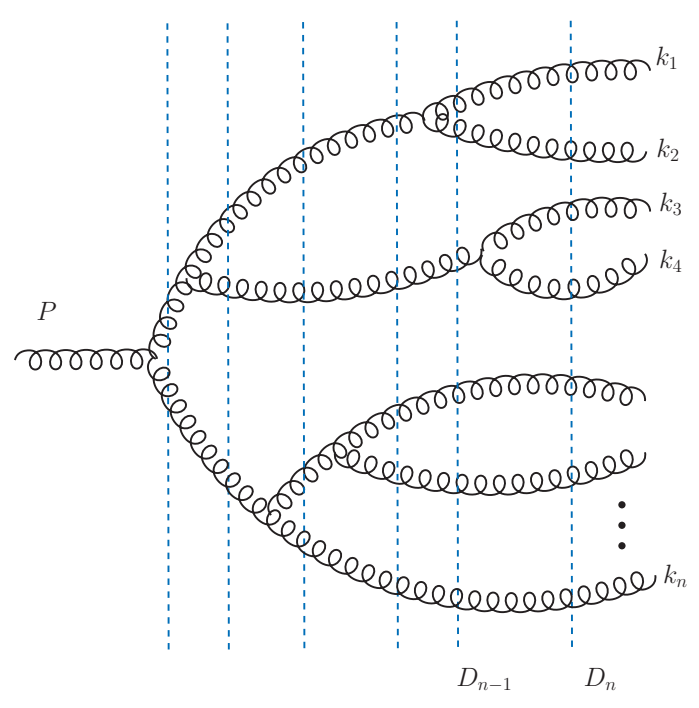

FIG. 1: The multi-gluon wave function. The vertical dashed lines symbolize different intermediate states where we need to evaluate the energy denominators. It is understood that the wave function scatters finally on some target.

To be specific, we start with a gluon with four momentum $P$, and the color index $a$, that develops a virtual fluctuation into states containing $n$ gluons with momenta $\left(k_{1}, \ldots k_{n}\right)$ and color indices $\left(a_{1}, a_{2}, \ldots, a_{n}\right)$, correspondingly see Fig. 1 . For the initial virtual gluon with virtuality $-Q^{2}$ and a vanishing transverse momentum one has in the light cone variables,

$$
P^{\mu}=\left(P^{+},-\frac{Q^{2}}{2 P^{+}}, \underline{0}\right)
$$

with $P^{ \pm}=\frac{1}{\sqrt{2}}\left(P^{0} \pm P^{3}\right)$. After $n-1$ splittings the wave function is shown in Fig. 1. The last $n$ gluons have the corresponding momenta labeled $k_{1}, \ldots, k_{n}$, as in Fig 1 . Each of this momenta has components $k_{i}^{\mu}=\left(z_{i} P^{+}, k_{i}^{-}, \underline{k}_{i}\right)$, with $z_{i}$ being the fraction of the initial $P^{+}$momentum which is carried by the gluon labeled by $i$ and $\underline{k}_{i}$ being the transverse component of the gluon momentum. The rules of the light cone perturbation theory [3, 4, 5, 6] require to evaluate the energy denominators for each of the intermediate states in Fig. 1 The energy denominator for the $n$ gluons in the intermediate state is defined to be a difference between the light cone energies of the initial and the intermediate state in question. For the wave function shown in Fig. 1 we assume the last intermediate state is with $n$ gluons as depicted. The corresponding energy denominator for this state reads

$$
\bar{D}_{n}=P^{-}-\sum_{i=1}^{n} k_{i}^{-}=-\frac{1}{2 P^{+}}\left(Q^{2}+\frac{k_{1}^{2}}{z_{1}}+\frac{k_{2}^{2}}{z_{2}}+\ldots+\frac{k_{n}^{2}}{z_{n}}\right)=-\frac{1}{2 P^{+}} D_{n}
$$


where we have used

$$
k_{i}^{-}=\frac{\underline{k}_{i}^{2}}{2 z_{i} P^{+}},
$$

and we introduced the auxiliary notation for the (rescaled) denominator $D_{n}$.

In what follows, we shall focus on the color ordered multi-gluon amplitudes, that is the amplitudes decomposed in the basis of color tensors, $T^{a_{1} a_{2} \ldots a_{n}}=\operatorname{tr}\left(t^{a_{1}} t^{a_{2}} \ldots t^{a_{n}}\right)$, where $a_{1}, a_{2}, \ldots, a_{n}$ are the color indices of the gluons. Up to the leading terms in the large $N_{c}$ approximation, these tensors are orthogonal, see e.g. 9] for a detailed discussion. This decomposition has a transparent graphical representation in 't Hooft's double line notation (see e.g. 61] for a review). The $n$-gluon amplitudes in the basis $T^{a_{1} a_{2} \ldots a_{n}}$ are then represented by planar diagrams built of the effective colored fermion lines. This, in turn, leads to the color dipole representation proposed by Mueller [1], in which the color coefficient in the triple gluon vertex is equal to unity. In what follows, we shall use the $n$-gluon components of the incoming particle light cone wave function $\Psi_{n}\left(k_{1}, k_{2}, \ldots, k_{n}\right)$, assuming implicitly, that the corresponding color tensor is given by the planar diagram in the double line notation, that corresponds to the definite ordering $\left(a_{1}, a_{2}, \ldots, a_{n}\right)$ of the gluon color indices.

\section{B. Recursion relations}

We focus on components of the wave function in which all the gluons have positive helicities. We work in the light-cone gauge, $\eta \cdot A=0$, with vector $\eta=(0,1, \underline{0})$ in the light-cone coordinates. This choice of gauge defines the polarization four-vectors of the gluon with four-momentum $k$

$$
\epsilon^{( \pm)}=\epsilon_{\perp}^{( \pm)}+\frac{\underline{\epsilon}^{( \pm)} \cdot \underline{k}}{\eta \cdot k} \eta
$$

where $\epsilon_{\perp}^{( \pm)}=\left(0,0, \underline{\epsilon}^{( \pm)}\right)$, and the transverse vector is defined by $\underline{\epsilon}^{( \pm)}=\mp \frac{1}{\sqrt{2}}(1, \pm i)$. The explicit projections of the triple and quartic gluon vertices show on this helicity states show that $[\underline{6}]$ :

1. The helicity transitions $(-\rightarrow++)$ and $(+\rightarrow--)$ are forbidden for the triple gluon vertex.

2. The $1 \rightarrow 3$ gluon transition given by the four-gluon vertex leads to mixed helicity composition of the three outgoing gluons.

These properties indicate, that the state after splitting always contains at least one gluon with the helicity of the parent. Thus, if we require the final state to be composed from gluons with positive helicities, there can be no gluons with negative helicities at any intermediate step. So, the four gluon-vertex does not contribute to the splitting process that we consider. In fact, the whole branching process is driven by a $(+\rightarrow++)$ helicity projection of the triple gluon vertex.

We define the projection of the triple gluon vertex on the $( \pm)$ polarization states, assuming that all the gluons are outgoing,

$$
\tilde{V}_{\lambda_{1} \lambda_{2} \lambda_{3}}^{a_{1} a_{2} a_{3}}\left(k_{1}, k_{2}, k_{3}\right)=\epsilon^{\left(\lambda_{1}\right) \mu_{1}} \epsilon^{\left(\lambda_{2}\right) \mu_{2}} \epsilon^{\left(\lambda_{3}\right) \mu_{3}} V_{\mu_{1} \mu_{2} \mu_{3}}^{a_{1} a_{2} a_{3}}\left(k_{1}, k_{2}, k_{3}\right)
$$

where $a_{1}, a_{2}, a_{3}$ are of course color indices. As we already stated, vertex $\tilde{V}_{\lambda_{1} \lambda_{2} \lambda_{3}}^{a_{1} a_{2} a_{3}}\left(k_{1}, k_{2}, k_{3}\right)$ vanishes if all the outgoing gluon helicities are the same, $\lambda_{1}=\lambda_{2}=\lambda_{3}$. When one helicity, say $\lambda_{1}=-1$, is different than the others $\lambda_{2}=\lambda_{3}=+1$, the vertex takes the following form in the light-cone variables,

$$
\tilde{V}_{-++}^{a_{1} a_{2} a_{3}}\left(k_{1}, k_{2}, k_{3}\right)=g f^{a_{1} a_{2} a_{3}} z_{1} \underline{\epsilon}^{(-)} \cdot\left(\frac{\underline{k}_{2}}{z_{2}}-\frac{\underline{k}_{3}}{z_{3}}\right)
$$

where the $\delta$-functions related to the conservation of the transverse and longitudinal ' + ' components of the momentum are implicit. Here $g$ is the strong coupling constant and $f^{a_{1} a_{2} a_{3}}$ is the structure constant for the $S U\left(N_{c}\right)$ color group. For the case of interest, of $(+\rightarrow++)$ transition, the amplitude is described by (6), with $z_{1}$ being the fraction of the + component of the momentum of the incoming gluon. Note that all dependence of the vertex on the transverse momenta of the daughter gluons is completely absorbed into a variable

$$
\underline{v}_{23} \equiv\left(\frac{\underline{k}_{2}}{z_{2}}-\frac{\underline{k}_{3}}{z_{3}}\right) .
$$


This variable is a relative transverse light cone velocity of the two gluons. Interestingly enough, the same variable is present when we consider the change of the energy denominator due to the splitting. In a general situation, when the gluon with momentum $k_{1}$ belongs to a virtual gluon cascade, the energy denominator before the splitting of gluon 1 can be written as

$$
D_{n}=D_{n / 1}+\frac{\underline{k}_{1}^{2}}{z_{1}}
$$

where $D_{n / 1}=Q^{2}+\sum_{i>1} \frac{\underline{k}_{i}^{2}}{z_{i}}$ does not contain the energy of gluon 1. Note that, we are using here the definition (3) for the energy denominator, which is different by the sign and with the $P^{+}$dependence factored out. After the gluon splits into two gluons with momenta $k_{2}$ and $k_{3}$ we have

$$
D_{n+1}=D_{n / 1}+\frac{\underline{k}_{2}^{2}}{z_{2}}+\frac{\underline{k}_{3}^{2}}{z_{3}} .
$$

In the light cone perturbation theory the transverse and the + components of the longitudinal momenta are conserved in the vertices therefore we have that that $\underline{k}_{1}=\underline{k}_{2}+\underline{k}_{3}$ and $z_{1}=z_{2}+z_{3}$. Using this fact one can express the change of the energy denominator as,

$$
D_{n+1}-D_{n}=\frac{z_{2} z_{3}}{z_{2}+z_{3}}\left(\frac{\underline{k}_{2}}{z_{2}}-\frac{\underline{k}_{3}}{z_{3}}\right)^{2} .
$$

It is convenient to introduce a variable that depends on the longitudinal degrees of freedom

$$
\xi_{23} \equiv \frac{z_{2} z_{3}}{z_{2}+z_{3}} .
$$

We therefore see that the change in the denominator (10) due to the splitting is expressed through the variable $v_{23}$ as well

$$
D_{n+1}-D_{n}=\xi_{23} \underline{v}_{23}^{2} .
$$

In the light-cone formulation of the QCD, the intermediate line that carries longitudinal momentum fraction $z_{i}$ is multiplied by $1 / \sqrt{z_{i}}$. It is therefore convenient to follow the convention by [6] and absorb such factors into all the gluon lines incoming and outgoing from the triple gluon vertex. Thus, we shall use

$$
\bar{V}_{\lambda_{1} \lambda_{2} \lambda_{3}}^{a_{1} a_{2} a_{3}}\left(k_{1}, k_{2}, k_{3}\right)=\frac{1}{\sqrt{z_{1} z_{2} z_{3}}} \tilde{V}_{\lambda_{1} \lambda_{2} \lambda_{3}}^{a_{1} a_{2} a_{3}}\left(k_{1}, k_{2}, k_{3}\right)=g f^{a_{1} a_{2} a_{3}} \frac{\underline{\epsilon}^{(-)} \underline{v}_{23}}{\sqrt{\xi_{23}}} .
$$

Following the discussion of the color structure of the amplitudes in Sec. IIA , in what follows we drop the structure constants $f^{a_{1} a_{2} a_{3}}$.

Now, we can collect the vertex and the energy denominator together to get the effect of the gluon splitting on the virtual multi-gluon state wave function. We shall introduce the following notation. Let $\Psi_{n-1}\left(k_{1}, k_{2}, \ldots, k_{i i+1}, \ldots, k_{n}\right)$ (with $\left.k_{i i+1} \equiv k_{i}+k_{i+1}\right)$ be the $(n-1)$-gluon wave function in momentum space before the splitting of gluon with momentum $k_{i+1}$, and $\Psi_{n}\left(k_{1}, k_{2}, \ldots, k_{i}, k_{i+1}, \ldots, k_{n}\right)$ the wave function after splitting of this gluon. Then the splitting of the gluon with momentum $k_{i i+1}$ acts as follows,

$$
\Psi_{n}\left(k_{1}, \ldots, k_{i}, k_{i+1}, \ldots, k_{n}\right)=\frac{g}{\sqrt{\xi_{i i+1}}} \frac{\underline{\epsilon}^{(-)} \underline{v}_{i i+1}}{D_{n-1}+\xi_{i i+1} \underline{v}_{i i+1}^{2}} \Psi_{n-1}\left(k_{1}, \ldots, k_{i i+1}, \ldots, k_{n}\right),
$$

where the color degrees of freedom are treated in the way described in Sec. IIIA.

The formula (14) is the recurrence prescription for obtaining the wave function with $n$ virtual gluons from the wave function with $n-1$ gluons. Of course to obtain the full recurrence formula one needs to sum over the different possibilities of the splittings which will give us the following result

$$
\Psi_{n}\left(k_{1}, k_{2}, \ldots, k_{n}\right)=\frac{g}{D_{n}} \sum_{i=2}^{n} \frac{\underline{\epsilon}^{(-)} \underline{\underline{v}}_{i-1 i}}{\sqrt{\xi_{i-1 i}}} \Psi_{n-1}\left(k_{1}, \ldots, k_{i-1 i}, \ldots, k_{n}\right),
$$

where $D_{n}$ is the denominator for the last intermediate state with $n$ gluons. The form of the splitting given by (14) and (15) looks highly symmetric, and all dependence on momenta of daughter gluons $i$ and $i-1$ is embedded into two variables: $\xi_{i-1 i}$ and $\underline{v}_{i-1 i}$. In this calculation we have kept the exact kinematics and therefore the energy denominator includes the full dependence on the momenta in the whole cascade. This is understandable, as in the case of the exact kinematics the new wave function has to carry the full information about the old wave function. We will come back to this point later in Sec. III when we discuss the high energy limit. 


\section{Relation to helicity amplitudes and the collinear limit in the on-shell case}

It turns out that the variables $\underline{v}_{j k}$ that we used to construct the wave functions in the previous subsection are related to the variables used in the framework of helicity amplitudes, see [9] for a nice review. Namely, for given pair of on-shell momenta $k_{i}$ and $k_{j}$ we have that

$$
\langle i j\rangle=\sqrt{z_{i} z_{j}} \underline{\epsilon}^{(+)} \cdot\left(\frac{\underline{k}_{i}}{z_{i}}-\frac{\underline{k}_{j}}{z_{j}}\right), \quad[i j]=\sqrt{z_{i} z_{j}} \underline{\epsilon}^{(-)} \cdot\left(\frac{\underline{k}_{i}}{z_{i}}-\frac{\underline{k}_{j}}{z_{j}}\right),
$$

where the symbols $[i j],\langle i j\rangle$ are the spinor products defined by

$$
\langle i \mid j\rangle=\langle i-\mid j+\rangle, \quad[i j]=\langle i+\mid j-\rangle .
$$

The chiral projections of the spinors for massless particles are defined as

$$
|i \pm\rangle=\psi_{ \pm}\left(k_{i}\right)=\frac{1}{2}\left(1 \pm \gamma_{5}\right) \psi\left(k_{i}\right), \quad\langle \pm i|=\overline{\psi_{ \pm}\left(k_{i}\right)}
$$

for a given momentum $k_{i}$. The spinor products are complex square roots of the total energy mass squared for the pair of gluons $(i, j)$

$$
\langle i j\rangle[i j]=\left(k_{i}+k_{j}\right)^{2}
$$

and they also satisfy $\langle i j\rangle=[i j]^{*}$. Using the above definitions (1619) we have that

$$
\langle i j\rangle[i j]=z_{i} z_{j}\left(\frac{\underline{k}_{i}}{z_{i}}-\frac{\underline{k}_{j}}{z_{j}}\right)^{2}
$$

which is real and positive for the on-shell gluon momenta. Finally, combining (7) and (16) we obtain

$$
\langle i j\rangle=\sqrt{z_{i} z_{j}} \underline{\epsilon}^{(+)} \cdot \underline{v}_{i j}, \quad[i j]=\sqrt{z_{i} z_{j}} \underline{\epsilon}^{(-)} \cdot \underline{v}_{i j},
$$

and the dependence on the transverse momenta in the light cone wave function can be expressed by $\langle i j\rangle$ and $[i j]$.

Using these expressions we can check the collinear limit for the on-shell case. The Eq. (14) is part of the recursion relation (15) for the off-shell multi-gluon wave function. It actually describes the situation in which the gluon with momentum $k_{12}$ splits into two daughter gluons, with momenta $k_{1}$ and $k_{2}$ respectively. It is interesting to investigate the collinear limit of the on-shell amplitude, which should get factorized. To get the on-shell amplitude one needs to drop the non-local denominator $D_{n-1}$ in (14). The factorizable limit for gluons 1 and 2 is then

$$
\begin{aligned}
& \Psi_{n}^{(1 \| 2)}\left(k_{1}, k_{2}, \ldots, k_{n}\right)=\frac{g}{\sqrt{\xi_{12}}} \frac{\underline{\epsilon}^{(-)} \underline{v}_{12}}{\xi_{12} \underline{v}_{12}^{2}} \Psi_{n-1}\left(k_{1}+k_{2}, k_{3}, \ldots, k_{n}\right)= \\
& =\frac{1}{\sqrt{\xi_{12}}} \frac{g}{\sqrt{z(1-z)}} \frac{[12]}{s_{12}} \Psi_{n-1}\left(k_{1}+k_{2}, k_{3}, \ldots, k_{n}\right)
\end{aligned}
$$

where $z=z_{1} /\left(z_{1}+z_{2}\right)$ and $s_{12}=\left(k_{1}+k_{2}\right)^{2}$. Relation (21) is, modulo $1 / \sqrt{\xi_{12}}$ coefficient and the sign convention, exactly the factorization relation on the collinear poles for the kinematical parts of the dual amplitudes as shown in [9]. It is interesting to note that the only thing that we have done here is to identify the gluons with momenta $k_{1}, k_{2}$ as originating from the splitting of the gluon with momentum $k_{12}$ and therefore we have selected only one splitting out of $n-2$ possible combinations.

\section{Multi-gluon wave function in the coordinate representation}

It is interesting to investigate the form of the recursion relation (14) in the transverse coordinate representation. By this we mean performing the Fourier transform with respect to the transverse components of the momenta, just like in the original dipole approach [1]. In this section we will consider a special case of the wave function where in the initial state we have only one gluon. In the original approach the initial state was the quark-antiquark pair. We will discuss the latter case in the next section where we will derive the modified dipole kernel. In this section we want 
to demonstrate calculational techniques which allow us to resum the consecutive splittings in the wave function. We therefore assume that initially we have the just one gluon, which has momentum $k_{1}$. The initial wave function in the transverse space is then written as follows

$$
\Phi_{1}(1) \equiv \Phi_{1}\left(z_{1}, \underline{r}_{1}\right)=\int \frac{d^{2} \underline{k}_{1}}{(2 \pi)^{2}} e^{i \underline{k}_{1} \cdot \underline{r}_{1}} \Psi_{1}\left(z_{1}, \underline{k}_{1}\right),
$$

where $\underline{r}_{1}$ is the coordinate in the transverse space. In the rest of the paper the symbol $\Psi$ will always denote the wave function in the momentum space, whereas $\Phi$ will denote the wave function in the coordinate space. For the wave function with $n$ gluons we define

$$
\begin{aligned}
\Phi_{n}(1, \ldots, n) \equiv \Phi_{n}\left(z_{1}, \underline{r}_{1} ; z_{2}, \underline{r}_{2} ; \ldots, z_{n}, \underline{r}_{n}\right) \\
=\int \frac{d^{2} \underline{k}_{1}}{(2 \pi)^{2}} \frac{d^{2} \underline{k}_{2}}{(2 \pi)^{2}} \ldots \frac{d^{2} \underline{k}_{n}}{(2 \pi)^{2}} \exp \left(i \underline{k}_{1} \cdot \underline{r}_{1}+i \underline{k}_{2} \cdot \underline{r}_{2}+\ldots+i \underline{k}_{n} \cdot \underline{r}_{n}\right) \Psi_{n}\left(k_{1}, \ldots, k_{n}\right),
\end{aligned}
$$

and similarly for $\Phi_{n-1}$ and $\Psi_{n-1}$. For the purpose of the subsequent calculation it will be convenient to change the variables for the transverse momenta from $\left(\underline{k}_{1}, \underline{k}_{2}\right)$ to $\underline{K}_{12}=\underline{k}_{1}+\underline{k}_{2}$ and $\underline{\kappa}_{12}=\xi_{12} \underline{v}_{12}$, and the longitudinal variables from $z_{1}, z_{2}$ to $z_{12}=z_{1}+z_{2}$, and $\xi_{12}$. These are actually the c.m.s. variables for gluons 1 and 2 . In addition one has: $d^{2} \underline{k}_{1} d^{2} \underline{k}_{2}=d^{2} \underline{K}_{12} d^{2} \underline{\kappa}_{12}$, and $z_{1} z_{2}=\xi_{12} z_{12}$. The scalar products can be rewritten as follows, $\underline{k}_{1} \underline{r}_{1}+\underline{k}_{2} \underline{r}_{2}=\underline{K}_{12} \underline{R}_{12}+\underline{\kappa}_{12} \underline{r}_{12}$, where $\underline{R}_{12}=\frac{z_{1} \underline{r}_{1}+z_{2} \underline{r}_{2}}{z_{1}+z_{2}}$, and $\underline{r}_{12}=\underline{r}_{2}-\underline{r}_{1}$. Next, we represent the energy denominator,

$$
\frac{1}{D_{n}}=\left[\frac{\underline{\kappa}_{12}^{2}}{\xi_{12}}+\frac{\underline{K}_{12}^{2}}{z_{12}}+\frac{\underline{k}_{3}^{2}}{z_{3}}+\ldots+\frac{\underline{k}_{n}^{2}}{z_{n}}+Q^{2}\right]^{-1}
$$

as

$$
\frac{1}{D_{n}}=\int_{0}^{\infty} d \tau \exp \left(-\tau D_{n}\right)
$$

where recall that $Q^{2}$ is the virtuality of the incoming particle. The above formula for the denominator (24) may be regarded as the Hamiltonian representation with the Euclidean time $\tau$. Using this representation we arrive at

$$
\begin{aligned}
& \Phi_{n}(1,2, \ldots, n)= \int \frac{d^{2} \underline{\kappa}_{12}}{(2 \pi)^{2}} \frac{d^{2} \underline{K}_{12}}{(2 \pi)^{2}} \ldots \frac{d^{2} \underline{k}_{n}}{(2 \pi)^{2}} \exp \left(i \underline{\kappa}_{12} \cdot \underline{r}_{12}+i \underline{K}_{12} \cdot \underline{R}_{12}+\ldots+i \underline{k}_{n} \cdot \underline{r}_{n}\right) \frac{\underline{\epsilon}^{(-)} \underline{v}_{12}}{\sqrt{\xi_{12}}} \\
& \times \int_{0}^{\infty} d \tau e^{-\tau D_{n}} \Psi_{n-1}\left(K_{12}, k_{3}, \ldots, k_{n}\right) \\
&=-i \frac{\underline{\epsilon}^{(-)} \cdot \underline{\partial}_{r_{12}}}{\xi_{12} \sqrt{\xi_{12}}} \int \frac{d^{2} \underline{\kappa}_{12}}{(2 \pi)^{2}} \frac{d^{2} \underline{K}_{12}}{(2 \pi)^{2}} \ldots \frac{d^{2} \underline{k}_{n}}{(2 \pi)^{2}} \exp \left(\underline{\kappa}_{12} \cdot \underline{r}_{12}+i \underline{K}_{12} \cdot \underline{R}_{12}+\ldots+i \underline{k}_{n} \cdot \underline{r}_{n}\right) \\
& \times \int_{0}^{\infty} d \tau \exp \left[-\tau\left(\frac{\underline{\kappa}_{12}^{2}}{\xi_{12}}+\frac{\underline{K}_{12}^{2}}{z_{12}}+\ldots+\frac{\underline{k}_{n}^{2}}{z_{n}}+Q^{2}\right)\right] \\
& \times \int d^{2} \underline{r}_{1}^{\prime} \ldots d^{2} \underline{r}_{n-1}^{\prime} \exp \left(-i\left(\underline{k}_{1}+\underline{k}_{2}\right) \cdot \underline{\underline{r}}_{1}^{\prime}-i \underline{k}_{3} \cdot \underline{r}_{2}^{\prime}-\ldots-i \underline{k}_{n} \cdot \underline{r}_{n-1}^{\prime}\right) \Phi_{n-1}\left(1^{\prime}, \ldots,(n-1)^{\prime}\right),
\end{aligned}
$$

where we have used the recursion formula (14) and the inverse Fourier transform for the $n$ gluon wave function. Note, that the primed $z$ values are the following: $z_{1}^{\prime}=z_{1}+z_{2}, z_{2}^{\prime}=z_{3}, \ldots, z_{n-1}^{\prime}=z_{n}$. The integrals over all transverse momenta are Gaussian and can be easily performed, which results in

$$
\begin{aligned}
& \Phi_{n}(1, \ldots, n)=-i \frac{\underline{\epsilon}^{(-)} \cdot \underline{\partial}_{r_{12}}}{\xi_{12} \sqrt{\xi_{12}}} \xi_{12} z_{12} z_{3} \ldots z_{n} \int_{0}^{\infty} d \tau\left(\frac{1}{4 \pi \tau}\right)^{n} \int d^{2} \underline{r}_{1}^{\prime} \ldots d^{2} \underline{r}_{n-1}^{\prime} \\
& \quad \times \exp \left[-\frac{1}{4 \tau}\left[\xi_{12} \underline{r}_{12}^{2}+z_{12}\left(\underline{r}_{1}^{\prime}-\underline{R}_{12}\right)^{2}+z_{3}\left(\underline{r}_{2}^{\prime}-\underline{r}_{3}\right)^{2}+\ldots+z_{n}\left(\underline{r}_{n-1}^{\prime}-\underline{r}_{n}\right)^{2}\right]-Q^{2} \tau\right] \Phi_{n-1}\left(1^{\prime}, 2^{\prime}, \ldots,(n-1)^{\prime}\right) .
\end{aligned}
$$

The integral over the 'time' $\tau$ can be performed to give the expression with the modified Bessel function $K_{0}$

$$
\Phi_{n}(1, \ldots, n)=i \frac{\underline{\epsilon}^{(-)} \cdot \underline{r}_{12}}{\sqrt{\xi_{12}}} z_{1} z_{2} \ldots z_{n} \int d^{2} \underline{r}_{1}^{\prime} \ldots d^{2} \underline{r}_{n-1}^{\prime} \Phi_{n-1}\left(1^{\prime}, 2^{\prime}, \ldots,(n-1)^{\prime}\right) \quad\left(-\frac{1}{\pi} \frac{\partial}{\partial A}\right)^{n} K_{0}\left(\sqrt{Q^{2} A}\right),
$$


where

$$
A \equiv \xi_{12} \underline{r}_{12}^{2}+z_{12}\left(\underline{r}_{1}^{\prime}-\underline{R}_{12}\right)^{2}+z_{3}\left(\underline{r}_{2}^{\prime}-\underline{r}_{3}\right)^{2}+\ldots+z_{n}\left(\underline{r}_{n-1}^{\prime}-\underline{r}_{n}\right)^{2} .
$$

The integral kernel $\left(-\frac{1}{\pi} \frac{\partial}{\partial A}\right)^{n} K_{0}\left(\sqrt{Q^{2} A}\right)$ can be rewritten into a more elegant form using the relations between the modified Bessel functions and their derivatives, see for example [62]

$$
\left(\frac{d}{x d x}\right)^{m}\left[x^{-n} K_{n}(x)\right]=(-1)^{m} x^{-n-m} K_{n+m}(x),
$$

which gives

$$
\left(-\frac{1}{\pi} \frac{\partial}{\partial A}\right)^{n} K_{0}\left(\sqrt{Q^{2} A}\right)=\frac{1}{(2 \pi)^{n}}\left(\frac{Q^{2}}{A}\right)^{\frac{n}{2}} K_{n}\left(\sqrt{Q^{2} A}\right)
$$

Using the above relations we can recast the recurrence relation (27) into

$$
\Phi_{n}(1, \ldots, n)=i \frac{\underline{\epsilon}^{(-)} \cdot \underline{r}_{12}}{\sqrt{\xi_{12}}} z_{1} z_{2} \ldots z_{n} \int \frac{d^{2} \underline{r}_{1}^{\prime} \ldots d^{2} \underline{r}_{n-1}^{\prime}}{(2 \pi)^{n}}\left(\frac{Q^{2}}{A}\right)^{\frac{n}{2}} K_{n}\left(\sqrt{Q^{2} A}\right) \Phi_{n-1}\left(1^{\prime}, 2^{\prime}, \ldots,(n-1)^{\prime}\right),
$$

with $A$ defined above (28). The formula (27) is the prescription for the off-shell tree level wave function with exact kinematics. Since it depends on the coordinates of all $n-1$ gluons through the variable $A$ defined above, it is quite complicated. Also, it should be kept in mind that this is just formula for one particular splitting, one needs to sum over all possible splittings like in (15). We will show nevertheless that in the case where the incoming particle is on-shell there are significant simplifications, which allow to resum the multiple gluon splittings. The crucial difference with respect to the leading logarithmic approximation with the Regge kinematics is the appearance of the modified Bessel functions $K_{n}$ which contain the information about the gluon splitting. In the original approach the splitting of the gluon leads to the expression which is just a power in the transverse coordinates. This translates into the power-like behavior of the splitting kernel in the dipole equation. Here, because the kinematics is kept exact the functional dependence is governed by the Bessel functions, which for large values of their arguments have exponential behavior asymptotically. This will result in a qualitative difference when investigating the impact parameter dependence of the scattering amplitude. We will come back to this problem and discuss it in more detail at the end of Sec. IV]

\section{E. Resumming the multi-gluon wave function in the case of the on-shell incoming gluon}

We consider here the multi-gluon wave function that originates from subsequent splittings of an on-shell incoming gluon with helicity + . One can also alternatively think about it as the incoming particle with a large momentum $P^{+}$such that $P^{-}=-\frac{Q^{2}}{2 P^{+}}$is very small, at least as compared with the particles in the wave function. This will result in energy denominators which do not contain the initial $P^{-}$. We will assume that all gluons have + helicities which should be a situation in the high energy limit, where the helicity flips are suppressed. We shall use the complex representation of the transverse vectors: $v_{i j}=\underline{\epsilon}^{(+)} \cdot \underline{v}_{i j}, v_{i j}^{*}=\underline{\epsilon}^{(-)} \cdot \underline{v}_{i j}$, and a useful notation,

$$
\begin{aligned}
& v_{\left(i_{1} i_{2} \ldots i_{p}\right)\left(j_{1} j_{2} \ldots j_{q}\right)}=\frac{k_{i_{1}}+k_{i_{2}}+\ldots+k_{i_{p}}}{z_{i_{1}}+z_{i_{2}}+\ldots+z_{i_{p}}}-\frac{k_{j_{1}}+k_{j_{2}}+\ldots+k_{j_{q}}}{z_{j_{1}}+z_{j_{2}}+\ldots+z_{j_{q}}}, \\
& \xi_{\left(i_{1} i_{2} \ldots i_{p}\right)\left(j_{1} j_{2} \ldots j_{q}\right)}=\frac{\left(z_{i_{1}}+z_{i_{2}}+\ldots+z_{i_{p}}\right)\left(z_{j_{1}}+z_{j_{2}}+\ldots+z_{j_{q}}\right)}{z_{i_{1}}+z_{i_{2}}+\ldots+z_{i_{p}}+z_{j_{1}}+z_{j_{2}}+\ldots+z_{j_{q}}},
\end{aligned}
$$

with notation $k_{i} \equiv \underline{\epsilon}^{(+)} \cdot \underline{k}_{i}$. The global momentum conservation $\delta$-functions, $\Delta^{(n)}=\delta^{(2)}\left(\sum_{i=1}^{n} \underline{k}_{i}\right) \delta\left(1-\sum_{i=1}^{n} z_{i}\right)$, that are present in all the expressions for the wave functions will not be displayed explicitly. Thus, the incoming state has the wave function,

$$
\Psi_{1}(1)=1 .
$$

In the following discussion we will consider color ordering in the amplitudes, therefore we will suppress color degrees of freedom. In general, for a color-ordered amplitude, the gluon splitting acts on the wave function as derived in Eq. (15) (for on-shell initial state though)

$$
-D_{n+1} \Psi_{n+1}(1,2, \ldots, n+1)=
$$




$$
=g \frac{v_{12}^{*}}{\sqrt{\xi_{12}}} \Psi_{n}(12,3, \ldots, n+1)+g \frac{v_{23}^{*}}{\sqrt{\xi_{23}}} \Psi_{n}(1,23, \ldots, n+1)+\ldots+g \frac{v_{n n+1}^{*}}{\sqrt{\xi_{n n+1}}} \Psi_{n}(1,2, \ldots, n n+1),
$$

with $D_{n+1}=\underline{k}_{1}^{2} / z_{1}+\underline{k}_{2}^{2} / z_{2}+\cdots+\underline{k}_{n+1}^{2} / z_{n+1}$. We have introduced the notation $\Psi_{n}(1, \ldots, i-1 i, \ldots, n+1)$ where $i-1 i$ means that it is the gluon with the momentum $k_{i-1 i}=k_{i-1}+k_{i}$. After the first splitting one gets

$$
\Psi_{2}(1,2)=-g \frac{1}{\sqrt{\xi_{12}}} \frac{v_{12}^{*}}{\xi_{12}\left|v_{12}\right|^{2}}=-g \frac{1}{\sqrt{\xi_{12}}} \frac{1}{\xi_{12} v_{12}}
$$

where we have taken that $P^{-}=0$. According to (33), the next splitting leads from $\Psi_{2}(1,2)$ to $\Psi_{3}(1,2,3)$ :

$$
\begin{aligned}
& -D_{3} \Psi_{3}(1,2,3)=-g\left[\frac{v_{12}^{*}}{\sqrt{\xi_{12}}} \Psi_{2}(12,3)+\frac{v_{23}^{*}}{\sqrt{\xi_{23}}} \Psi_{2}(1,23)\right] \\
& =g^{2}\left[\frac{v_{12}^{*}}{\sqrt{\xi_{12} \xi_{(12) 3}}} \frac{1}{\xi_{(12) 3} v_{(12) 3}}+\frac{v_{23}^{*}}{\sqrt{\xi_{23} \xi_{1(23)}}} \frac{1}{\xi_{1(23)} v_{1(23)}}\right] .
\end{aligned}
$$

This expression may be, after some simple algebra, simplified using $\xi_{12} \xi_{(12) 3}=\xi_{23} \xi_{1(23)}=\frac{z_{1} z_{2} z_{3}}{z_{1}+z_{2}+z_{3}}=z_{1} z_{2} z_{3}$. One obtains,

$$
\Psi_{3}(1,2,3)=g^{2} \frac{1}{\sqrt{z_{1} z_{2} z_{3}}} \frac{1}{\xi_{(12) 3} \xi_{1(23)}} \frac{1}{v_{(12) 3} v_{1(23)}}
$$

Note that, the energy denominator $D_{3}$ disappeared from the equation as it has canceled with the numerator when finding the common denominator for expression (35). The same procedure can be iterated further. We shall give below the explicit form of the wave function obtained for 4 gluons and then present a generalization to an arbitrary $n$. Thus, for $n=4$ we found:

$$
\Psi_{4}(1,2,3,4)=-g^{3} \frac{1}{\sqrt{z_{1} z_{2} z_{3} z_{4}}} \frac{1}{\xi_{(123) 4} \xi_{(12)(34)} \xi_{1(234)}} \frac{1}{v_{(123) 4} v_{(12)(34)} v_{1(234)}},
$$

and for a general integer $n>2$ one expects,

$$
\begin{aligned}
\Psi_{n}(1,2, \ldots, n)=( & -1)^{n-1} g^{n-1} \frac{1}{\sqrt{z_{1} z_{2} \ldots z_{n}}} \frac{1}{\xi_{(12 \ldots n-1) n} \xi_{(12 \ldots n-2)(n-1 n) \ldots \xi_{1(2 \ldots n)}}} \\
& \times \frac{1}{v_{(12 \ldots n-1) n} v_{(12 \ldots n-2)(n-1 n) \cdots v_{1(2 \ldots n)}}}
\end{aligned}
$$

This formula was explicitly verified for $n=2,3,4,5,6$. The proof for arbitrary $n$ can be done by mathematical induction and proceeds as follows. We assume that the wave function $\Psi_{n}$ satisfies the above conjecture (38). Using (33) the wave function $\Psi_{n+1}$ has then the form

$$
\begin{aligned}
& -D_{n+1} \Psi_{n+1}(1,2, \ldots, n+1)=g \sum_{i=1}^{n} \frac{v_{i i+1}^{*}}{\sqrt{\xi_{i i+1}}} \Psi_{n}(1,2, \ldots,(i i+1), \ldots, n+1)= \\
& (-1)^{n-1} g^{n} \sum_{i=1}^{n} \frac{v_{i i+1}^{*}}{\sqrt{\xi_{i i+1}}} \frac{1}{\sqrt{z_{1} z_{2} \ldots\left(z_{i}+z_{i+1}\right) \ldots z_{n}}} \frac{1}{\left(\xi_{(12 \ldots n) n+1} \xi_{\left.(12 \ldots n-1)(n n+1) \ldots \xi_{1(2 \ldots n+1)}\right)^{\prime}}\right.} \times \\
& \frac{1}{\left(v_{(12 \ldots n) n+1} v_{(12 \ldots n-1)(n n+1)} \ldots v_{1(2 \ldots n+1)}\right)^{\prime}} \text {. }
\end{aligned}
$$

We have inserted the symbol' to denote the fact that the indices $i i+1$ have to be taken together, or in other words in each term of the sum in (39) for a given $i$ the denominator does not have the term of the form $\xi_{(1 \ldots i)(i+1 \ldots n+1)} v_{(1 \ldots i)(i+1 \ldots n+1)}$. The expression under the square root is

$$
\xi_{i i+1} z_{1} z_{2} \ldots\left(z_{i}+z_{i+1}\right) \ldots z_{n}=\frac{z_{i} z_{i+1}}{z_{i}+z_{i+1}} z_{1} z_{2} \ldots\left(z_{i}+z_{i+1}\right) \ldots z_{n}=z_{1} z_{2} \ldots z_{n+1}
$$


because $i$ goes from 1 to $n$, there are $n+1$ terms in the product (40). Now we write (39) in a form with the common denominator for all the terms which gives

$$
\begin{gathered}
D_{n+1} \Psi_{n+1}(1,2, \ldots, n+1)=(-1)^{n} g^{n} \frac{\sum_{i=1}^{n} v_{i i+1} \xi_{(1 \ldots i)(i+1 \ldots n+1)} v_{(1 \ldots i)(i+1 \ldots n+1)}}{\sqrt{z_{1} z_{2} \ldots z_{n+1}} \xi_{(12 \ldots n) n+1} \xi_{(12 \ldots n-1)(n n+1) \ldots \xi_{1(2 \ldots n+1)}}} \times \\
\frac{1}{v_{(12 \ldots n) n+1} v_{(12 \ldots n-1)(n n+1)} \ldots v_{1(2 \ldots n+1)}}
\end{gathered}
$$

The denominator in the above equation is just what gives the wave function $\Psi_{n+1}$. An essential point is to show that

$$
\sum_{i=1}^{n} v_{i i+1} \xi_{(1 \ldots i)(i+1 \ldots n+1)} v_{(1 \ldots i)(i+1 \ldots n+1)}=\sum_{j=1}^{n+1} \frac{\underline{k}_{j}^{2}}{z_{j}} \equiv D_{n+1}
$$

that is that the numerator on the right hand side in (41) cancels $D_{n+1}$ on the left hand side of this equation. We notice that,

$$
v_{(1 \ldots i)(i+1 \ldots n)} \xi_{(1 \ldots i)(i+1 \ldots n)}=\left(\frac{k_{1}+\ldots+k_{i}}{z_{1}+\ldots+z_{i}}+\frac{k_{1}+\ldots k_{i}}{1-\left(z_{1}+\ldots+z_{i}\right)}\right) \xi_{(1 \ldots i)(i+1 \ldots n)}=\sum_{j=1}^{i} k_{j} .
$$

Therefore the left hand side of (42) is

$$
\begin{aligned}
& \sum_{i=1}^{n} v_{i i+1} \xi_{(1 \ldots i)(i+1 \ldots n+1)} v_{(1 \ldots i)(i+1 \ldots n+1)}=\sum_{i=1}^{n} v_{i i+1} \sum_{j=1}^{i} k_{j}=\sum_{i=1}^{n}\left(\frac{k_{i}}{z_{i}}-\frac{k_{i+1}}{z_{i+1}}\right) \sum_{j=1}^{i} k_{j}= \\
&=\sum_{i=1}^{n} \frac{k_{i}}{z_{i}} \sum_{j=1}^{i} k_{j}-\sum_{i=1}^{n} \frac{k_{i+1}}{z_{i+1}} \sum_{j=1}^{i} k_{j}=\sum_{i=1}^{n} \frac{k_{i}^{2}}{z_{i}}+\sum_{i=2}^{n} \frac{k_{i}}{z_{i}} \sum_{j=1}^{i-1} k_{j}+\frac{k_{n+1}^{2}}{z_{n+1}}-\sum_{i=1}^{n-1} \frac{k_{i+1}}{z_{i+1}} \sum_{j=1}^{i} k_{j} .
\end{aligned}
$$

The second and fourth term cancel, and the first and third give

$$
\sum_{i=1}^{n+1} \frac{k_{i}^{2}}{z_{i}}=D_{n+1}
$$

which is exactly the denominator, so we have proven (42). Note that, we have also used

$$
\sum_{i=1}^{n} k_{i}=-k_{n+1}
$$

i.e. the condition which is coming from the energy-momentum conservation. This completes the proof, as $\Psi_{n+1}$ is exactly of the form (38) for $n \rightarrow n+1$.

The resummed form (38) looks relatively simple. Its dependence on transverse momenta of gluons factorized into reciprocals of $v_{(12 \ldots p)(p \ldots n)}$ with the splitting index $p$ takes all possible positions from $p=2$ to $p=n-1$. The wave function given by (38) may be also expressed in terms of the $\langle i j\rangle$ symbols in the following way:

$$
\begin{aligned}
& \Psi_{n}(1,2, \ldots, n)=(-1)^{n-1} g^{n-1} \frac{1}{\sqrt{z_{1} z_{2} \ldots z_{n}}} \frac{1}{\sqrt{\xi_{(12 \ldots n-1) n} \xi_{(12 \ldots n-2)(n-1 n) \cdots \xi_{1(2 \ldots n)}}}} \\
& \frac{1}{\langle(12 \ldots n-1) n\rangle\langle(12 \ldots n-2)(n-1 n)\rangle \ldots\langle 1(2 \ldots n)\rangle} .
\end{aligned}
$$

The wave function given by (38) can be simplified further.

Denoting $k_{(1 \ldots p)} \equiv k_{1}+\ldots+k_{p}$ we may write:

$$
\Psi_{n}(1,2, \ldots, n)=(-1)^{n-1} g^{n-1} \frac{1}{\sqrt{z_{1} z_{2} \ldots z_{n}}} \frac{1}{k_{(1)} k_{(12)} \ldots k_{(12 \ldots n-1)}} .
$$


One may go back to the real representation of the transverse vectors and perform the Fourier transform of (47),

$$
\Phi_{n}\left(z_{1}, \underline{r}_{1} ; \ldots ; z_{n}, \underline{r}_{n}\right)=\int \frac{d^{2} \underline{k}_{1}}{(2 \pi)^{2}} \ldots \frac{d^{2} \underline{k}_{n}}{(2 \pi)^{2}} \exp \left(i \underline{k}_{1} \cdot \underline{r}_{1}+\ldots+i \underline{k}_{n} \cdot \underline{r}_{n}\right) \Psi_{n}\left(z_{1}, \underline{k}_{1}, \ldots, z_{n}, \underline{k}_{n}\right) .
$$

It is convenient to change the momentum variables:

$$
\left\{\underline{k}_{1}, \underline{k}_{2}, \ldots \underline{k}_{n}\right\} \rightarrow\left\{\underline{k}_{(1)}, \underline{k}_{(12)}, \ldots, \underline{k}_{(12 \ldots n)}\right\}
$$

The Jacobian of the transformation is trivial, and one expresses the gluon momenta in the following way:

$$
\underline{k}_{1}=\underline{k}_{(1)}, \quad \underline{k}_{2}=\underline{k}_{(12)}-\underline{k}_{(1)}, \quad \ldots, \quad \underline{k}_{n}=\underline{k}_{(12 \ldots n)}-\underline{k}_{(12 \ldots n-1)} .
$$

The Fourier exponent takes the form,

$$
\exp \left(i \underline{k}_{1} \cdot \underline{r}_{1}+\ldots+i \underline{k}_{n} \cdot \underline{r}_{n}\right)=\exp \left(i \underline{k}_{(1)} \cdot\left(\underline{r}_{1}-\underline{r}_{2}\right)+i \underline{k}_{(12)} \cdot\left(\underline{r}_{2}-\underline{r}_{3}\right)+\ldots \underline{k}_{(12 \ldots n-1)} \cdot\left(\underline{r}_{n-1}-\underline{r}_{n}\right)+i \underline{k}_{(12 \ldots n)} \cdot \underline{r}_{n}\right)
$$

and the final answer for the wave function in position space reads

$$
\Phi_{n}\left(z_{1}, \underline{r}_{1} ; \ldots ; z_{n}, \underline{r}_{n}\right)=(-1)^{n-1} g^{n-1} \delta\left(1-\sum_{i=1}^{n} z_{i}\right) \frac{1}{\sqrt{z_{1} z_{2} \ldots z_{n}}} \frac{\underline{\epsilon}^{(-)} \underline{r}_{12} \underline{\epsilon}^{(-)} \underline{r}_{23} \cdots \underline{\epsilon}^{(-)} \underline{r}_{n-1 n}}{r_{12}^{2} r_{23}^{2} \ldots r_{n-1 n}^{2}} .
$$

Surprisingly, this is precisely the structure of the gluon wave function in the LL dipole model. However, it is crucial for the derivation that the incoming gluon is on-shell, and that we chose a very special component of the wave function, with all positive gluon helicities. Note that the on-shellness (or quasi-onshellness) of the incoming particle means that in the light cone perturbation theory we neglect in the energy denominators the energy of this particular particle and keep only the energies of the subsequent emitted gluons.

\section{DIPOLE KERNEL WITH APPROXIMATE KINEMATICS}

\section{A. Dipole wave-function in the leading logarithmic approximation.}

In the previous section we have presented a general approach for the treatment of the light cone wave function while keeping the kinematics exact. We were able to construct the recurrence relation for the off-shell case and resum the wave function for the special case of the on-shell incoming particle and with all the gluons having the same helicities. However, for the practical purposes, it would be desirable to construct the evolution equation in rapidity that would resum the gluon emissions just like in the original dipole approach developed by Mueller [1]. We will show in this section that it is possible, though not all the exact kinematical effects are kept here. The modified equation should include at least part of the corrections due to the exact kinematics. These corrections will prove to be sufficient to recover the double logarithmic corrections found in the next-to-leading calculation.

Let us therefore recall the original construction of the dipole wave function at small $x$ with soft gluons which was first developed by Mueller [1]. Unlike the previous section, where we took the initial particle to be the gluon we will consider here the $q \bar{q}$ pair in the color singlet state, which is the onium. The heavy onium wave function, which consists of the quark-antiquark pair without any additional gluons, is defined as

$$
\Psi^{(0)}\left(z_{1}, \underline{k}_{1}\right), \quad \text { where } \quad z_{1}=k_{1}^{+} / P^{+} .
$$

The initial momentum is $P$, the quark has four-momentum $k_{1}=\left(k_{1}^{+}, k_{1}^{-}, \underline{k}_{1}\right)$ and the antiquark has momentum $P-k_{1}$. In the transverse space the wave-function reads

$$
\Phi^{(0)}\left(z_{1}, \underline{x}_{01}\right)=\int \frac{d^{2} \underline{k}_{1}}{(2 \pi)^{2}} e^{i \underline{k}_{1} \cdot \underline{x}_{01}} \Psi^{(0)}\left(z_{1}, \underline{k}_{1}\right)
$$

where $\underline{x}_{01}$ is the size of the dipole 01 in the transverse space. Strictly speaking, this representation is a mixed one: coordinate space in the transverse components and momentum space in the longitudinal degrees of freedom.

Such representation was shown [1] to be very convenient for the purpose of studying the high energy limit, as only in this limit there is a decoupling of the transverse and longitudinal degrees of freedom. Let us investigate the emission 
of one gluon with momentum $k_{2}$, see Fig. 2. If we were to keep the kinematics exact we would arrive at the analog of the formula (14) for the case of one splitting, where say the gluon is emitted from the upper anti-quark line (Fig. (2)

$$
\Psi_{\text {exact }}^{(1)}\left(z_{1}, \underline{k}_{1} ; z_{2}, \underline{k}_{2}\right)=\frac{g t^{a} \bar{u}\left(k_{1}\right) \gamma \cdot \epsilon_{2} u\left(k_{1}+k_{2}\right)}{\bar{D}_{1}} \Psi^{(0)}\left(z_{1}, \underline{k}_{1}\right) .
$$

Here the exact energy denominator has the form

$$
\bar{D}_{1}=P^{-}-\left[\left(P-k_{1}-k_{2}\right)^{-}+k_{1}^{-}+k_{2}^{-}\right],
$$

and similarly for the second emission from the other quark line. Following the procedure presented in the previous section one would obtain the recursion formulae which depend on the kinematics of the whole cascade. The simplification that arises in the high energy limit is due to the fact that the emission of the very soft daughter gluon gets factorized from the rest of the wave function. In other words there is no recoil due to the emission of the gluon and the rest of the whole cascade is frozen and is not affected by the splitting. This allows to factorize the emissions of the gluons and resum their emissions in the form of the evolution equation which is differential in rapidity. We shall show that the leading logarithmic approach will be changed when the more exact kinematics is taken into account, but the resummation of the emission can be still recast in the form of the evolution equation. To this aim let us recall the original assumptions made in [1] that lead to the derivation of the dipole evolution equation in the leading logarithmic approximation.

The following assumptions are done in order to reproduce the leading logarithmic approximation, or the limit of the high energy

- The emitted gluon is longitudinally soft: $k_{2}^{+} \ll k_{1}^{+}$, and one defines $z_{1}=k_{1}^{+} / P^{+}, z_{2}=k_{2}^{+} / P^{+}$which are the fractions of the longitudinal momenta. Obviously $z_{2} \ll z_{1}$.

- The coupling of the gluon to the quark (antiquark) is eikonal

$$
g t^{a} \bar{u}\left(k_{1}\right) \gamma \cdot \epsilon_{2} u\left(k_{1}+k_{2}\right)=2 g t^{a} k_{1} \cdot \epsilon_{2},
$$

where $t^{a}$ is the color matrix in the fundamental representation and $\epsilon_{2}$ is the polarization vector of the emitted gluon with momentum $k_{2}$.

- Since the gluon is longitudinally soft $k_{2}^{+} \ll k_{1}^{+}$one can keep in graphs the leading term in the energy denominator (see Fig. 2)

$$
\bar{D}_{1}=\frac{1}{P^{-}-\left[\left(P-k_{1}-k_{2}\right)^{-}+k_{1}^{-}+k_{2}^{-}\right]} \simeq \frac{1}{k_{2}^{-}},
$$

because $k_{2}^{-}$is the dominant term $\left(k_{2}^{-}=\underline{k}_{2}^{2} / 2 k_{2}^{+}\right.$and $k_{2}^{+}$is very small, $\left.k_{2}^{+} \ll k_{1}^{+}, P^{+}\right)$. Here, $P$ is the initial momentum, see Fig. 2.
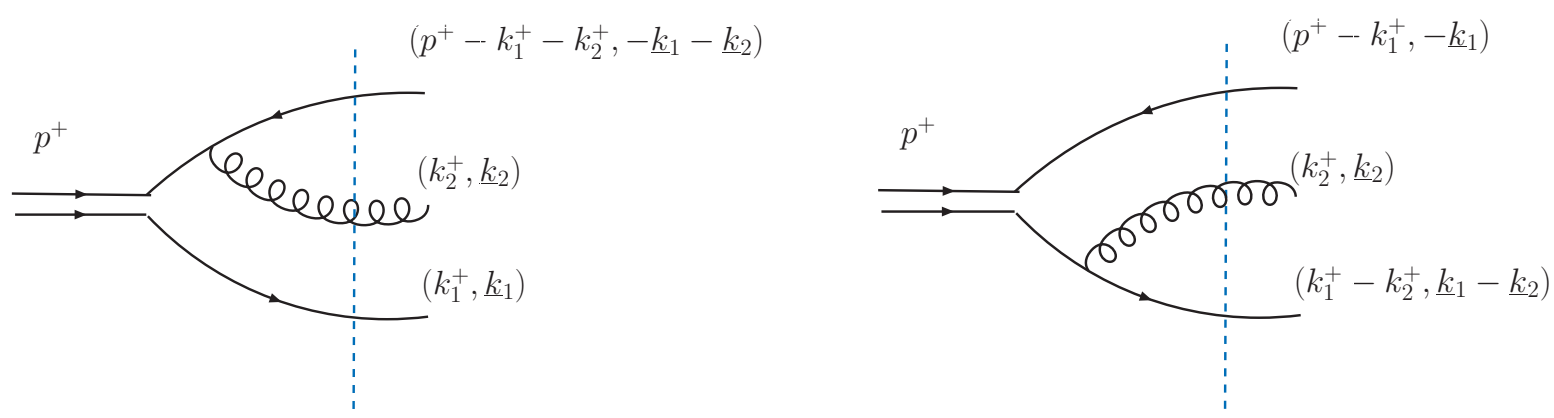

FIG. 2: Dipole wave function with one soft gluon. Dashed line indicates the energy denominator for the intermediate state.

If one puts together all these assumptions one arrives at the formula for the wave function with one emitted gluon

$$
\Psi^{(1)}\left(z_{1}, \underline{k}_{1} ; z_{2}, \underline{k}_{2}\right)=2 g t^{a} \frac{\underline{\epsilon}_{2} \cdot \underline{k}_{2}}{\underline{k}_{2}^{2}}\left[\Psi^{(0)}\left(z_{1}, \underline{k}_{1}\right)-\Psi^{(0)}\left(z_{1}, \underline{k}_{1}+\underline{k}_{2}\right)\right] \Theta\left(z_{1}-z_{2}\right) .
$$


At this stage there is only ordering in the longitudinal fractions $z_{1} \gg z_{2}$ which is extended to the weak inequality $z_{1} \geq z_{2}$ in the subsequent calculation. The transverse momenta are unrestricted in this approximation. One can perform the Fourier transform of the above expression to obtain the formula for the wave function with one soft gluon in the transverse space

$$
\Phi^{(1)}\left(z 1, \underline{x}_{01} ; z_{2}, \underline{x}_{02}\right)=\int \frac{d^{2} \underline{k}_{1}}{(2 \pi)^{2}} \frac{d^{2} \underline{k}_{2}}{(2 \pi)^{2}} e^{i \underline{k}_{1} \cdot \underline{x}_{01}+i \underline{k}_{2} \cdot \underline{x}_{02}} \Psi^{(1)}\left(z_{1}, \underline{k}_{1} ; z_{2}, \underline{k}_{2}\right),
$$

which yields the explicit result

$$
\Phi^{(1)}\left(z_{1}, \underline{x}_{01} ; z_{2}, \underline{x}_{02}\right)=-\frac{i g t^{a}}{\pi}\left(\frac{\underline{x}_{20}}{x_{20}^{2}}-\frac{\underline{x}_{21}}{x_{21}^{2}}\right) \cdot \underline{\epsilon}_{2} \Psi^{(0)}\left(z_{1}, \underline{x}_{01}\right)
$$

where $x_{i j}^{2} \equiv \underline{x}_{i j}^{2}$. We see the advantage of using the coordinate space representation because the soft gluons factorize in (59) as demonstrated in [1]. The modulus squared of the one gluon wave function has then explicitly the form

$$
\left|\Phi^{(1)}\right|^{2}\left(z_{1}, \underline{x}_{01}\right)=\int_{z_{0}}^{z_{1}} \frac{d z_{2}}{z_{2}} \int \frac{d^{2} \underline{x}_{02}}{2 \pi} \frac{\underline{x}_{01}^{2}}{\underline{x}_{02}^{2} \underline{x}_{12}^{2}}\left|\Phi^{(0)}\left(z_{1}, \underline{x}_{01}\right)\right|^{2} .
$$

In this form it is particularly transparent that in the soft gluon limit the transverse and longitudinal degrees of freedom decouple. Therefore the transverse space coordinate representation is especially useful. The wave function with one soft gluon is just the wave function without any soft gluons times the branching probability. The measure (or branching probability) in this case reads

$$
\frac{d^{2} \underline{x}_{02} \underline{x}_{01}^{2}}{\underline{x}_{02}^{2} \underline{x}_{12}^{2}}
$$

is the dipole splitting kernel in the leading logarithmic approximation which appears in the dipole evolution equation for the dipole scattering amplitude and it was originally derived in 1]. The same kernel appears in nonlinear version of the evolution equation with additional gluon rescatterings: the Balitsky-Kovchegov equation [17, 20]. It is straightforward to check that this kernel is invariant with respect to the 2-dimensional conformal transformations. In deriving the equation (57) one uses crucial assumption about the strong ordering in the energies

$$
k_{2}^{-} \gg k_{1}^{-}
$$

In the original approach [1] one uses the assumption of the softness of the gluon with respect to the parent dipole and treats the transverse momenta as unordered which is equivalent to the Regge kinematics. This enables to make the approximations as described above and factorize the soft gluon contribution. However, for the consistency of the calculations we should keep the inequality (62) (where $k_{1}^{-}=\frac{\underline{k}_{1}^{2}}{2 k_{1}^{+}}, k_{2}^{-}=\frac{\underline{k}_{2}^{2}}{2 k_{2}^{+}}$) exact which gives

$$
\frac{\underline{k}_{2}^{2}}{k_{2}^{+}}>\frac{\underline{k}_{1}^{2}}{k_{1}^{+}}
$$

with $k_{1}^{+}>k_{2}^{+}$.

If there are more gluon emissions we will of course have

$$
\ldots \frac{\underline{k}_{i_{4}}^{2}}{k_{i_{4}}^{+}}>\frac{\underline{k}_{i_{3}}^{2}}{k_{i_{3}}^{+}}>\frac{\underline{k}_{i_{2}}^{2}}{k_{i_{2}}^{+}}>\frac{\underline{k}_{i_{1}}^{2}}{k_{i_{1}}^{+}},
$$

and

$$
\cdots<k_{i_{4}}^{+}<k_{i_{3}}^{+}<k_{i_{2}}^{+}<k_{i_{1}}^{+}
$$

where the indices $i_{1}, \ldots, i_{4}$ enumerate subsequent emissions along one branch of the gluon cascade.

As a result of the ordering (63) the region in the transverse momenta $\underline{k}_{2}^{2}$ for the gluon emission is limited

$$
\Theta\left(\underline{k}_{2}^{2}-\underline{k}_{1}^{2}\right)+\Theta\left(\underline{k}_{1}^{2}-\underline{k}_{2}^{2}\right) \Theta\left(\underline{k}_{2}^{2}-\underline{k}_{1}^{2} \frac{k_{2}^{+}}{k_{1}^{+}}\right) .
$$


This means that there is a constraint which restricts the transverse momenta of the daughter gluon (labeled 2). which is the step function $\Theta\left(\underline{k}_{2}^{2}-\underline{k}_{1}^{2} \frac{k_{2}^{+}}{k_{1}^{+}}\right)$. The constraint (6365) means that the momenta of the emitted gluons (those with $k_{2}$ ) are cutoff in the infrared. Note that, the ordering (63) is exactly the same as the one discussed in 663, 64, 65], it is ordering in the fluctuation time. One has to think about the cascade as developing from the hadron side and the onium as a model of a hadron.

Given the discussion above, the wave function of the onium with one soft gluon should be modified to include the constraint (63) and with this modification reads

$$
\Psi^{(1)}\left(z_{1}, \underline{k}_{1} ; z_{2}, \underline{k}_{2}\right)=2 g t^{a} \frac{\underline{\epsilon}_{2} \cdot \underline{k}_{2}}{\underline{k}_{2}^{2}}\left[\Psi^{(0)}\left(z_{1}, \underline{k}_{1}\right)-\Psi^{(0)}\left(z_{1}, \underline{k}_{1}+\underline{k}_{2}\right)\right] \Theta\left(z_{1}-z_{2}\right) \Theta\left(\frac{\underline{k}_{2}^{2}}{z_{2}}-\frac{\underline{k}_{1}^{2}}{z_{1}}\right),
$$

where we used $z_{1}$ and $z_{2}$ instead of $k_{1}^{+}$and $k_{2}^{+}$. At this point it is no longer so trivial to perform the Fourier transform into the coordinate space since the transverse momenta are entangled now and the soft gluons do not factorize as before in coordinate space. In particular, there will be a shift (or distortion) of the original dipole since we encounter integrals of the type (if we choose to make the $\underline{k}_{1}$ integral first in the following example )

$$
\int \frac{d^{2} \underline{k}_{1}}{(2 \pi)^{2}} e^{i \underline{k}_{1} \cdot \underline{x}_{01}} \Psi^{(0)}\left(z_{1}, \underline{k}_{1}\right) \Theta\left(\frac{\underline{k}_{2}^{2}}{z_{2}}-\frac{\underline{k}_{1}^{2}}{z_{1}}\right) \text {. }
$$

Therefore we see that the kinematical constraint emerges in the light-cone perturbation theory from the more exact treatment of the energy denominators in the graphs. In the $t$-channel formulation of the BFKL Pomeron, the analogous consistency constraint arises, when one takes into account the fact that the virtualities of the exchanged gluons are dominated by the transverse parts. This leads to the constraint on the transverse momenta of the emitted gluons, see for example [40].

\section{B. Modified energy denominators in the dipole evolution}

In the previous section we have seen how the kinematical constraint emerges from the more careful treatment of the energy denominators. In this section we will demonstrate that one can include this effect into the dipole wave function. In particular we will arrive at a modified dipole evolution equation. Let us take the more exact version of the energy denominator which includes the energy of the parent emitter

$$
\frac{1}{k_{1}^{-}+k_{2}^{-}} \text {. }
$$

With this modification the formula (57) for the dipole wave function in momentum space with one gluon becomes

$$
\Psi^{(1)}\left(z_{1}, \underline{k}_{1} ; z_{2}, \underline{k}_{2}\right)=2 g t^{a} \frac{\underline{\epsilon}_{2} \cdot \underline{k}_{2}}{\underline{k}_{2}^{2}+\underline{k}_{1}^{2} \frac{k_{2}^{+}}{k_{1}^{+}}}\left[\Psi^{(0)}\left(z_{1}, \underline{k}_{1}\right)-\Psi^{(0)}\left(z_{1}, \underline{k}_{1}+\underline{k}_{2}\right)\right] .
$$

We still keep the vertex to be eikonal, the only modifications are in the energy denominator. Let us define the scale

$$
\bar{Q}^{2} \equiv \underline{k}_{1}^{2} \frac{k_{2}^{+}}{k_{1}^{+}}=\underline{k}_{1}^{2} z, z=\frac{z_{2}}{z_{1}},
$$

and perform the two-dimensional Fourier transform of [68) to the coordinate space

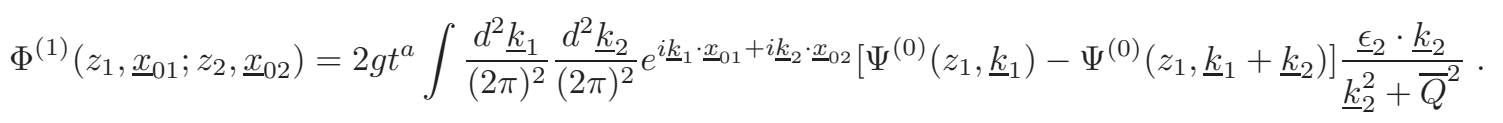

Let us take the first term in the above formula

$$
\begin{aligned}
2 g t^{a} \int \frac{d^{2} \underline{k}_{1}}{(2 \pi)^{2}} \frac{d^{2} \underline{k}_{2}}{(2 \pi)^{2}} e^{i \underline{k}_{1} \cdot \underline{x}_{01}+i \underline{k}_{2} \cdot \underline{x}_{02} \Psi^{(0)}\left(\underline{k}_{1}, z_{1}\right) \frac{\underline{\epsilon}_{2} \cdot \underline{k}_{2}}{\underline{k}_{2}^{2}+\bar{Q}^{2}}=} \\
=2 g t^{a} \int \frac{d^{2} \underline{k}_{1}}{(2 \pi)^{2}} e^{i \underline{k}_{1} \cdot \underline{x}_{01}} \Psi^{(0)}\left(\underline{k}_{1}, z_{1}\right) \frac{i}{2 \pi} \bar{Q} K_{1}\left(\bar{Q} x_{02}\right) \frac{\underline{\epsilon}_{2} \cdot \underline{x}_{02}}{x_{02}} \\
=2 g t^{a} \int d^{2} \underline{r} \Phi^{(0)}(\underline{r}, z) \int \frac{d^{2} \underline{k}_{1}}{(2 \pi)^{2}} e^{i \underline{k}_{1} \cdot\left(\underline{x}_{01}-\underline{r}\right)} \frac{i}{2 \pi} \bar{Q} K_{1}\left(\bar{Q} x_{02}\right) \frac{\underline{\epsilon}_{2} \cdot \underline{x}_{02}}{x_{02}} .
\end{aligned}
$$


To get the last line, we have used the inverse Fourier transform to represent the wave function in the coordinate space $\Phi^{(0)}(z, \underline{r})$. Since the expression with the modified Bessel function $\bar{Q} K_{1}\left(\bar{Q} x_{02}\right)$ depends on $\underline{k}_{1}$ we do not get a delta function $\delta^{(2)}\left(\underline{r}-\underline{x}_{01}\right)$ after the integration over $\underline{k}_{1}$. This means that the parent dipole recoils or changes its size because of the emission of the daughter dipole. If we insist that the recoil is small we can still make an improvement over the original LL dipole formula by making the following approximations

$$
\bar{Q}_{01} \simeq \frac{1}{x_{01}} \sqrt{\frac{k_{2}^{+}}{k_{1}^{+}}}=\frac{1}{x_{01}} \sqrt{z},
$$

and after performing the $k_{1}$ integration which in this approximation gives delta function we finally get

$$
2 g t^{a} \Phi^{(0)}\left(z, \underline{x}_{01}\right) \frac{i}{2 \pi} \bar{Q}_{01} K_{1}\left(\bar{Q}_{01} x_{02}\right) \frac{\underline{\epsilon}_{2} \cdot \underline{x}_{02}}{x_{02}} .
$$

This is an amplitude for the one gluon emission in the coordinate space improved by taking into account the next, subleading term in the energy denominator. Note, that it is a simplified and special version of (29). The difference is that we do not keep all the terms in the energy denominators but only the ones which are related to the 'parent' emitter and the last gluon in the emission. This allows to factorize the splitting from the rest of the cascade. Therefore in this case each emission will be governed by the same kernel (73) whereas in the exact case the splitting is governed by more complicated expression with the Bessel function whose order changes with the number of the gluons in the wave function.

The last expression (73) obviously reduces to the original LL dipole formula, compare (59), by expanding the Bessel function $K_{1}$ for the small values of the argument

$$
\bar{Q}_{01} K_{1}\left(\bar{Q}_{01} x_{02}\right)=\frac{\sqrt{z}}{x_{01}} K_{1}\left(\frac{x_{02}}{x_{01}} \sqrt{z}\right) \simeq \frac{1}{x_{02}}, \text { for } \frac{x_{02}}{x_{01}} \sqrt{z} \rightarrow 0 .
$$

The expression (73) becomes in this limit

$$
2 g t^{a} \Phi^{(0)}\left(\underline{x}_{01}, z\right) \frac{i}{2 \pi} \frac{\underline{\epsilon}_{2} \cdot \underline{x}_{02}}{x_{02}^{2}}
$$

which is the original LL formula [1] as expected. Therefore, (73) is an improvement over the original formula (75). Note that, there is a similarity of (73) with the form of the dipole formula for the $F_{2}$ structure function

$$
F_{T, L}\left(x, Q^{2}\right)=\frac{Q^{2}}{4 \pi^{2} \alpha_{\mathrm{em}}} \int d^{2} \mathbf{r} \int_{0}^{1} d z\left|\Psi_{T, L}^{\gamma^{*}, q \bar{q}}\left(r, z, Q^{2}\right)\right|^{2} \hat{\sigma}(r, x),
$$

where $\Psi_{T, L}^{\gamma^{*}, q \bar{q}}$ is the wave function for the splitting of the virtual photon into a $q \bar{q}$ pair (dipole), and $\hat{\sigma}$ is the imaginary part of the forward scattering amplitude of the $q \bar{q}$ dipole on the proton, called the dipole cross section, which describes the interaction of the dipole with the proton. In addition, $\mathbf{r}$ is the transverse separation of the quarks in the $q \bar{q}$ pair, and $z$ is the light-cone momentum fraction of the photon carried by the quark (or antiquark). As usual, $-Q^{2}$ is the photon virtuality and $x$ is the Bjorken variable defined as $x=Q^{2} / s$ with $s$ the total energy of the $\gamma^{*} p$ system. The wave function of the virtual photon is given by the following equations:

$$
\begin{aligned}
\left|\Psi_{T}^{\gamma^{*}, q \bar{q}}\right|^{2} & =\frac{3 \alpha_{e m}}{2 \pi^{2}} \sum_{f} e_{f}^{2}\left\{\left[z^{2}+(1-z)^{2}\right] \bar{Q}_{f}^{2} K_{1}^{2}\left(\bar{Q}_{f} r\right)+m_{f}^{2} K_{0}^{2}\left(\bar{Q}_{f} r\right)\right\} \\
\left|\Psi_{L}^{\gamma^{*}, q \bar{q}}\right|^{2} & =\frac{3 \alpha_{e m}}{2 \pi^{2}} \sum_{f} e_{f}^{2}\left\{4 Q^{2} z^{2}(1-z)^{2} K_{0}^{2}\left(\bar{Q}_{f} r\right)\right\}
\end{aligned}
$$

where the sum is performed over quarks with flavor $f$, charge $e_{f}$, and

$$
\bar{Q}_{f}^{2}=z(1-z) Q^{2}+m_{f}^{2} .
$$

We see that whenever $z$ or $1-z$ are small the wave-function can be approximated by

$$
\bar{Q}_{f}^{2} K_{1}^{2}\left(\bar{Q}_{f} r\right) \simeq \frac{1}{r^{2}},
$$


in a close analogy with the dipole kernel in the LL approximation, compare the square of (75). We see that (73) contains the kinematical constraint in the transverse space which is realized by the exponential tail of the Bessel function for large values of its argument

$$
\bar{Q}_{01} K_{1}\left(\bar{Q}_{01} x_{02}\right)=\frac{\sqrt{z}}{x_{01}} K_{1}\left(\frac{x_{02}}{x_{01}} \sqrt{z}\right) \simeq \sqrt{\frac{\pi \sqrt{z}}{2 x_{02} x_{01}}} \exp \left(-\frac{x_{02}}{x_{01}} \sqrt{z}\right), \quad \frac{x_{02}}{x_{01}} \sqrt{z} \rightarrow \infty,
$$

We have deliberately chosen the notation of $\bar{Q}$ variable in (69) to make contact with the analogous variable in the wave function of the photon $\bar{Q}^{2} \equiv Q^{2} z(1-z)$ (77/78). Of course we still have here small $z$, so that $(1-z) \simeq 1$.

The improved dipole kernel coming from squaring (73) plus the term from the second graph, reads

$$
\begin{aligned}
d^{2} \underline{x}_{2}\left(\bar{Q}_{01} K_{1}\left(\bar{Q}_{01} x_{02}\right) \frac{\underline{\epsilon}_{2} \cdot \underline{x}_{02}}{x_{02}}\right. & \left.-\bar{Q}_{01} K_{1}\left(\bar{Q}_{01} x_{12}\right) \frac{\underline{\epsilon}_{2} \cdot \underline{x}_{12}}{x_{12}}\right)^{2}= \\
= & d^{2} \underline{x}_{2} \bar{Q}_{01}^{2}\left[K_{1}^{2}\left(\bar{Q}_{01} x_{02}\right)+K_{1}^{2}\left(\bar{Q}_{01} x_{12}\right)-2 K_{1}\left(\bar{Q}_{01} x_{02}\right) K_{1}\left(\bar{Q}_{01} x_{12}\right) \frac{\underline{x}_{02} \cdot \underline{x}_{12}}{x_{02} x_{12}}\right],
\end{aligned}
$$

with

$$
\bar{Q}_{01}=\frac{1}{x_{01}} \sqrt{z}
$$

compare Eq. (172). We will refer to it as the quasilocal case because in this approximation we are keeping only terms in the energy denominators which refer to the daughter and the parent dipole, without any other dipoles in the cascade. It is straightforward to verify that the (80) simplifies to (61) when $z \rightarrow 0$. The kernel (80) is very similar in form to the one with the massive gluon, which also can be expressed in terms of the Bessel functions. Here, however the argument of the Bessel functions depends on the $\bar{Q}_{01}$, and consequently on the longitudinal momentum $z$, see (81). The transverse and longitudinal momenta are not separated any more, even though we can still use a single, closed integral equation for the evolution of the dipole amplitude in the rapidity. In the LL approximation the evolution depended only on the previous step in rapidity, with the branching that was independent of the rapidity or $x$. The modified kernel (80) contains the branchings which depend explicitly on the longitudinal variable, and therefore on all the steps in the evolution in rapidity. This is a qualitative difference as this means that there is now a 'memory' in the evolution of the system of dipoles. The probability of the emission of next dipoles depends on the evolution variable ('time') $z$.

\section{Soft gluon emission with the hard gluon displacement}

The approximation that we made in deriving (80) preserved an important feature of the soft gluon limit: the variation of the emitter gluon position in the process of soft gluon emission is neglected. Thus, transverse position of the harder of the two daughter gluons coincides with the transverse position of the parent gluon. A more accurate treatment of the emission process shows, however, that it is not the case. In order to demonstrate is explicitly, we go back to expression (71) with $\bar{Q}=\underline{k}_{1} \sqrt{z}$,

$$
2 g t^{a} \int d^{2} \underline{r} \Phi^{(0)}(\underline{r}, z) \int \frac{d^{2} \underline{k}_{1}}{(2 \pi)^{2}} e^{\left.i \underline{k}_{1} \cdot \underline{x}_{01}-\underline{r}\right)} \frac{i}{2 \pi} \bar{Q} K_{1}\left(\bar{Q} x_{02}\right) \frac{\underline{\epsilon}_{2} \cdot \underline{x}_{02}}{x_{02}}
$$

and perform the integral over $d^{2} \underline{k}_{1}$ to get,

$$
\begin{aligned}
2 g t^{a} \int d^{2} \underline{r} \Phi^{(0)}(\underline{r}, z) \int \frac{k_{1} d k_{1}}{2 \pi} J_{0}\left(k_{1}\left|\underline{x}_{01}-\underline{r}\right|\right) \frac{i}{2 \pi} k_{1} \sqrt{z} K_{1}\left(k_{1} \sqrt{z} x_{02}\right) \frac{\underline{\epsilon}_{2} \cdot \underline{x}_{02}}{x_{02}}= \\
=2 g t^{a} \int d^{2} \underline{r} \Phi^{(0)}(\underline{r}, z) \frac{\underline{\epsilon}_{2} \cdot \underline{x}_{02}}{x_{02}} \frac{i}{2 \pi} \sqrt{z} \int \frac{k_{1}^{2} d k_{1}}{2 \pi} J_{0}\left(k_{1}\left|\underline{x}_{01}-\underline{r}\right|\right) K_{1}\left(k_{1} \sqrt{z} x_{02}\right)= \\
\quad=2 g t^{a} \int d^{2} \underline{r} \Phi^{(0)}(\underline{r}, z) \frac{\underline{\epsilon}_{2} \cdot \underline{x}_{02}}{x_{02}^{2}} \frac{i}{(2 \pi)^{2}} \frac{2\left(\sqrt{z} x_{02}\right)^{2}}{\left[\left(\underline{x}_{01}-\underline{r}\right)^{2}+\left(\sqrt{z} x_{02}\right)^{2}\right]^{2}} .
\end{aligned}
$$

The last term on the r.h.s. is a Cauchy type distribution in two dimensions which gives delta function $\delta^{(2)}\left(\underline{x}_{01}-\underline{r}\right)$ when the scale parameter $\sqrt{z} x_{02} \rightarrow 0$. In this limit one reproduces expression (75). Last line of (83) gives more a 
precise expression in the situation when the change of the emitter gluon position is taken into account. Note, that the integration in (83) over the transverse vector $\underline{r}$ occurs already at the amplitude level, so the evolution equation for the dipole scattering amplitude with this kernel would involve a cumbersome triple integration over transverse coordinates. Finally, let us conclude that in a general case of the gluon splitting, the transverse position of neither of the daughter gluons coincides with the position of the parent gluon.

\section{Modified dipole evolution kernel and the NLL BFKL kernel}

In the previous subsection we proposed an approximate scheme to go beyond the soft gluon limit in the description of the onium wave function. In this scheme one better accounts for the kinematical effects in the evolution of the gluon wave function. A significant part of the phase space for the gluon emissions is cut out. The effect is of course subleading with respect to the leading logarithmic approximation or the original dipole cascade. Nevertheless, the constraint introduces important corrections and cannot be neglected in the analysis of the color dipole / BFKL evolution beyond the LL approximation. In particular, we shall demonstrate explicitly that in the NLL approximation the modified dipole kernel (80) saturates the double collinear logarithmic contributions that are found in the NLL BFKL kernel. As discussed in [41] these contributions violate the renormalization group constraints and should be resummed. The resummation of these spurious terms within the conventional $t$-channel formulation of the BFKL evolution in the momentum space is conveniently realized by imposing the so called kinematical constraint (or the consistency constraint) [38, 39, 40]. The modified dipole kernel (80) and the BFKL kernel with the kinematic constraint have similar origin and describe the same effect, a modification of the evolution kernel due to a more accurate treatment of the kinematical effects. In what follows, we shall discuss in detail the problem of the double collinear logarithms in the dipole representation.

In the exact next-to-leading calculation [10], 11, 12, 13, 14] the terms with the largest power of the collinear logarithms come from the following part of the NLL BFKL kernel in the coordinate representation,

$$
\mathcal{K}_{\mathrm{DL}}^{\mathrm{NLO}} \otimes N_{Y}=-2 \bar{\alpha}_{s}^{2} \int \frac{d^{2} \underline{x}_{2}}{2 \pi} \frac{x_{01}^{2}}{x_{02}^{2} x_{12}^{2}} \log \left(\frac{x_{02}}{x_{01}}\right) \log \left(\frac{x_{12}}{x_{01}}\right)\left[N_{Y}\left(\underline{x}_{0}, \underline{x}_{2}\right)+N_{Y}\left(\underline{x}_{1}, \underline{x}_{2}\right)-N_{Y}\left(\underline{x}_{0}, \underline{x}_{1}\right)\right],
$$

where $N_{Y}\left(\underline{x}_{i}, \underline{x}_{j}\right)$ in the above equation is the scattering amplitude of the color dipole $\left(\underline{x}_{i}, \underline{x}_{j}\right)$ with the target and $Y=\ln 1 / x$ is the rapidity between the incoming dipole and the target particle. It is important to note that these double logarithmic terms are the same in the QCD as in the conformally invariant $\mathcal{N}=4$ SYM theory [1]. Thus, we conjecture that this universality originates from universal kinematical effects in gluon emission.
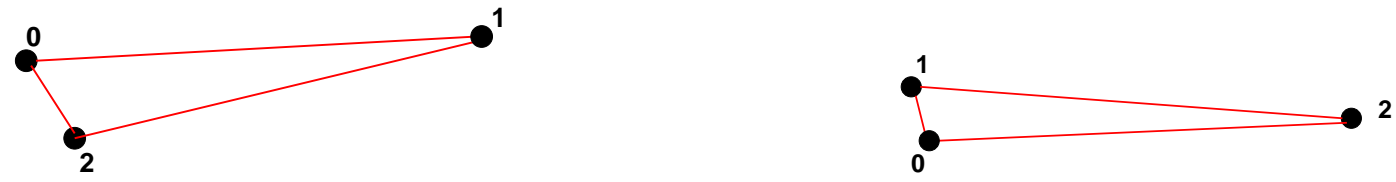

FIG. 3: Dipole configurations in two collinear limits. Left plot: $x_{02} \ll x_{01}$ and $x_{12} \sim x_{01}$; right plot: $x_{02} \gg x_{01}$ and $x_{12} \sim$ $x_{02}$.

Let us compare the behavior of (80) and (84) in the collinear and the anti-collinear limits. These are limits when the parent and daughter dipole sizes are strongly ordered. In the anti-collinear limit one of the daughter dipoles is much smaller than the parent dipole and we have,

$$
\left(x_{02} \ll x_{01} \text { and } x_{12} \sim x_{01}\right) \text { or }\left(x_{12} \ll x_{01} \text { and } x_{02} \sim x_{01}\right) \text {. }
$$

This situation is depicted on the left plot in Fig. 3. In these configurations no super-leading logarithmic enhancement of NLL BFKL kernel occurs, in particular the part of the NLL kernel (84) is suppressed as

$$
\log \frac{x_{12}}{x_{01}} \simeq \log \frac{x_{01}}{x_{01}} \rightarrow 0 \quad \text { or } \quad \log \frac{x_{02}}{x_{01}} \simeq \log \frac{x_{01}}{x_{01}} \rightarrow 0 .
$$

Thus, no anti-collinear double logarithms are generated. In the collinear limit both daughter dipoles are larger than the parent dipole. This configuration is shown in the right plot in Fig. 3

$$
x_{02} \gg x_{01} \text { and } x_{12} \sim x_{02} .
$$


In this case the most singular part (84) of the next-to-leading kernel tends to

$$
\mathcal{K}_{\mathrm{DL}}^{\mathrm{NLO}} \otimes N_{Y} \rightarrow-\frac{\bar{\alpha}_{s}^{2}}{\pi} \int \frac{d^{2} \underline{x}_{2} x_{01}^{2}}{x_{02}^{4}} \log ^{2}\left(\frac{x_{02}}{x_{01}}\right)[\ldots],
$$

where [...] stands for the expression in the square brackets as in (84).

Note that kernel (84) is large and negative in the regime where the produced dipoles $\underline{x}_{02}$ and $\underline{x}_{12}$ are very large compared to the dipole $x_{01}$. This is exactly the same regime where the modified kernel (80) is exponentially cutting off the contributions of the large (02), (12) dipoles. Clearly, in such configuration the phenomenologically improved kernel (80) is better behaved than the NLL kernel.

In the case of the kinematic constraint in the BFKL equation in momentum space,the double collinear logarithmic term is robust. These terms are entirely determined by the dependence of the effective cut-off scale for the transverse momentum on the longitudinal momentum fraction and does not depend on the details of the cut-off procedure. The same property should hold true in the position space. Thus, for the purpose of extraction of the contribution enhanced by the double collinear logarithms, it is sufficient to use a simplified dipole kernel

$$
\frac{x_{01}^{2}}{x_{02}^{2} x_{12}^{2}} \theta\left(x_{01}^{2}-z x_{02}^{2}\right) \theta\left(x_{01}^{2}-z x_{12}^{2}\right) .
$$

This kernel introduces the cut-off on large dipole sizes consistent with the effective cut-offs given by the more accurate expression (80), but it is more convenient to analyze. The physical meaning of this kernel is simple: if the daughter dipole size is small enough, the deviations from the LL kernel of the kinematic origin are small, and if the dipole sizes are too large, the kinematical effects introduce a distortion, which leads to a suppression of the emission. This suppression, in particular, is visible in the Bessel improved kernel.

The integral evolution equation for the dipole scattering amplitude with this kernel reads,

$$
N_{01}(x)=N_{01}^{(0)}+\bar{\alpha}_{s} \int_{x}^{1} \frac{d z}{z} \frac{d^{2} x_{2}}{2 \pi} \frac{x_{01}^{2}}{x_{02}^{2} x_{12}^{2}} \theta\left(z x_{01}^{2}-x x_{02}^{2}\right) \theta\left(z x_{01}^{2}-x x_{12}^{2}\right)\left[N_{02}(z)+N_{21}(z)-N_{01}(z)\right] .
$$

We shall go to the differential form w.r.t. the longitudinal variable $x$. One gets,

$$
\begin{gathered}
-x \frac{\partial}{\partial x} N_{01}(x)=\bar{\alpha}_{s} \int \frac{d^{2} x_{2}}{2 \pi} \frac{x_{01}^{2}}{x_{02}^{2} x_{12}^{2}}\left\{\theta\left(x_{01}^{2}-x_{02}^{2}\right) \theta\left(x_{01}^{2}-x_{12}^{2}\right)\left[N_{02}(x)+N_{21}(x)-N_{01}(x)\right]\right. \\
+\frac{\bar{\alpha}_{s}}{2 \pi} \int_{x}^{1} \frac{d z}{z}\left[\delta\left(x-z \frac{x_{01}^{2}}{x_{02}^{2}}\right) \theta\left(z x_{01}^{2}-x x_{12}^{2}\right)+\delta\left(x-z \frac{x_{01}^{2}}{x_{12}^{2}}\right) \theta\left(z x_{01}^{2}-x x_{02}^{2}\right)\right] \\
\left.\times\left[N_{02}(z)+N_{21}(z)-N_{01}(z)\right]\right\} .
\end{gathered}
$$

Clearly, due to limitations in the $x_{2}$ integration imposed by the $\theta$ functions, the first line does not introduce the double logarithms. Let us focus on the part of the equation containing the integration over $z$. This gives, after the $z$ integration

$$
\begin{gathered}
\int d^{2} x_{2} \frac{x_{01}^{2}}{x_{02}^{2} x_{12}^{2}}\left\{\theta\left(x_{02}^{2}-x_{01}^{2}\right) \theta\left(x_{02}^{2}-x_{12}^{2}\right)\left[N_{02}\left(x \frac{x_{02}^{2}}{x_{01}^{2}}\right)+N_{21}\left(x \frac{x_{02}^{2}}{x_{01}^{2}}\right)-N_{01}\left(x \frac{x_{02}^{2}}{x_{01}^{2}}\right)\right]+\right. \\
\left.\theta\left(x_{12}^{2}-x_{01}^{2}\right) \theta\left(x_{12}^{2}-x_{02}^{2}\right)\left[N_{02}\left(x \frac{x_{12}^{2}}{x_{01}^{2}}\right)+N_{21}\left(x \frac{x_{12}^{2}}{x_{01}^{2}}\right)-N_{01}\left(x \frac{x_{12}^{2}}{x_{01}^{2}}\right)\right]\right\} .
\end{gathered}
$$

Being interested in the NLL accuracy we may expand the dipole scattering amplitudes with the shifted $x$ argument, e.g.

$$
N_{i j}\left(x x_{02}^{2} / x_{01}^{2}\right)
$$

using a logarithmic variable $\log (x)$,

$$
N_{i j}\left(x x_{02}^{2} / x_{01}^{2}\right) \simeq N_{i j}(x)+x \frac{\partial}{\partial x} N_{i j}(x) \log \left(\frac{x_{02}^{2}}{x_{01}^{2}}\right) .
$$


The terms $N_{i j}(x)$ (without the differentiation w.r.t. $x$ ) may be combined with the first line of (87) to recover the LL dipole emission kernel. The emerging correction to the evolution equation takes the form,

$$
\begin{gathered}
{[\delta K \otimes N]_{01}(x)=\frac{\bar{\alpha}_{s}}{2 \pi} \int d^{2} x_{2} \frac{x_{01}^{2}}{x_{02}^{2} x_{12}^{2}} \times} \\
\left\{\theta\left(x_{02}^{2}-x_{01}^{2}\right) \theta\left(x_{02}^{2}-x_{12}^{2}\right) \log \left(\frac{x_{02}^{2}}{x_{01}^{2}}\right) x \frac{\partial}{\partial x}\left[N_{02}(x)+N_{21}(x)-N_{01}(x)\right]+\right. \\
\left.\theta\left(x_{12}^{2}-x_{01}^{2}\right) \theta\left(x_{12}^{2}-x_{02}^{2}\right) \log \left(\frac{x_{12}^{2}}{x_{01}^{2}}\right) x \frac{\partial}{\partial x}\left[N_{02}(x)+N_{21}(x)-N_{01}(x)\right]\right\} .
\end{gathered}
$$

Next, we shall use the BFKL equation to eliminate the $x$-differential, $x \partial / \partial x N_{i j}(x)$,

$$
x \frac{\partial}{\partial x} N_{i j}(x)=-\frac{\bar{\alpha}_{s}}{2 \pi} \int d^{2} x_{k} \frac{x_{i j}^{2}}{x_{i k}^{2} x_{k j}^{2}}\left[N_{i k}(x)+N_{k j}(x)-N_{i j}(x)\right] .
$$

Let us evaluate the action of the complete non-leading part of the expanded kernel on a probe function $N_{i j}^{(\gamma)}(x)=$ $n(x)\left(x_{i j}^{2}\right)^{\gamma}$, for $\gamma \rightarrow 1$. In this regime, the action of the LO order BFKL (dipole) kernel gives obviously,

$$
K \otimes N_{i j}^{(\gamma)}(x) \simeq \frac{\bar{\alpha}_{s}}{1-\gamma} N_{i j}^{(\gamma)}(x)
$$

This expression is inserted in (91). First, we evaluate

$$
-\frac{\bar{\alpha}_{s}}{2 \pi} \int d^{2} x_{2} \frac{x_{01}^{2}}{x_{02}^{2} x_{21}^{2}} \theta\left(x_{02}^{2}-x_{01}^{2}\right) \theta\left(x_{02}^{2}-x_{12}^{2}\right) \log \left(\frac{x_{02}^{2}}{x_{01}^{2}}\right) \frac{\bar{\alpha}_{s}}{1-\gamma}\left[N_{02}^{(\gamma)}(x)+N_{21}^{(\gamma)}(x)-N_{01}^{(\gamma)}(x)\right]
$$

for $\gamma \rightarrow 1$. The leading singularity at $\gamma=1$, is determined by the asymptotically large dipole sizes, $x_{02}^{2}=r^{2} \gg x_{01}^{2}$ and $x_{12}^{2} \simeq r^{2} \gg x_{01}^{2}$. Thus, the integral may be approximated by

$$
-\frac{\bar{\alpha}_{s}}{2 \pi} \int_{x_{01}^{2}}^{\infty} \frac{\pi}{2} d r^{2} \frac{x_{01}^{2}}{r^{4}} \log \left(\frac{r^{2}}{x_{01}^{2}}\right) \frac{2 \bar{\alpha}_{s}}{1-\gamma} r^{2 \gamma}=-x_{01}^{2 \gamma} \frac{\bar{\alpha}_{s}^{2}}{(1-\gamma)^{3}},
$$

where we took into account the effect of the constraint imposed by the function $\theta\left(x_{02}^{2}-x_{12}^{2}\right)$. Since $\underline{x}_{12}=\underline{x}_{02}-\underline{x}_{01}$, and $\left|\underline{x}_{02}\right| \gg\left|\underline{x}_{01}\right|$, this $\theta$ function constrains $\underline{x}_{12}$ to a half-plane for which the angle between $\underline{x}_{02}$ and $\underline{x}_{01}$ is smaller than $\pi / 2$. Therefore the angular part of the integration $d^{2} x_{2}$ gives only $\pi$ and not $2 \pi$. The same contribution as the one computed in (95) comes from the term proportional to $\theta\left(x_{12}^{2}-x_{01}^{2}\right) \theta\left(x_{12}^{2}-x_{02}^{2}\right)$ in (91), and the full answer reads,

$$
\left[\delta K \otimes N^{(\gamma)}\right]_{01}(x)=-\frac{\bar{\alpha}_{s}^{2}}{(1-\gamma)^{3}} N_{01}^{(\gamma)}(x)+\mathcal{O}\left(\frac{\bar{\alpha}_{s}^{2}}{(1-\gamma)^{2}}\right)
$$

This is precisely the same leading collinear singularity as the one found in the exact NLL kernel (84), 11, 12, 13, 14].

Finally, let us comment on the issue of the conformal invariance of the NLL BFKL kernel. The part of NLL kernel given by (84) is scale invariant. This means that the rescaling transformation $\underline{x}_{i} \rightarrow C \underline{x}_{i}$ leaves this part unchanged for an arbitrary number $C$. It is, however, straightforward to verify that $\mathcal{K}_{\mathrm{DL}}^{\mathrm{NL}} \overline{\mathrm{O}}$ is not conformally invariant in two transverse dimensions, for instance, the action of the kernel changes under the inversion $\underline{x}_{i} \rightarrow \frac{\underline{x}_{i}}{x_{i}^{2}}$. Interestingly enough, $\mathcal{K}_{\mathrm{DL}}^{\mathrm{NLO}}$ is the only part of the NLL BFKL kernel in $\mathcal{N}=4 \mathrm{SYM}$ theory that is non-conformal (of course in the QCD, already the scale invariance of the NLL BFKL/dipole kernel is broken by the running coupling effects). As mentioned before the modified dipole kernel (80) is also non-conformal in two dimensions since the arguments of the Bessel-McDonald function are exactly the same dipole size ratios $\frac{x_{02}}{x_{01}}, \frac{x_{12}}{x_{01}}$ as in the above next-to-leading contribution (84). 


\section{E. Diffusion in impact parameter space}

As is clear from the form of the modified kernel (80), the corrections from the energy denominators imply large modifications of the diffusion properties in the impact parameter space. The modified Bessel functions exponentially suppress the production of large size dipoles above a $z$-dependent characteristic size

$$
\bar{Q}_{01}^{2} K_{1}^{2}\left(\bar{Q}_{01} x_{02}\right) \simeq \frac{\pi}{2} \frac{\bar{Q}_{01}}{x_{02}} e^{-2 x_{02} \bar{Q}_{01}}=\frac{\pi}{2} \frac{\sqrt{z}}{x_{02} x_{01}} e^{-2 \frac{x_{02}}{x_{01}} \sqrt{z}}, \quad \bar{Q}_{01} x_{02} \rightarrow \infty .
$$

The effective cut-off size $\sim x_{01} / \sqrt{z}$ grows with the decreasing $z$. This cutoff on the dipole size is analogous to the coherence effect in the cascade of the gluon emissions [64]. The maximal opening angle prevents the gluons from being emitted into a certain kinematic regime. Here, the effect is to prevent the emission of very large dipoles and as a result the diffusion in the impact parameter space is very much suppressed.

To illustrate the diffusion better we inspect the Balitsky-Kovchegov equation with the LL and the modified kernel respectively. We would like to stress however that the fact that we are using the modified dipole kernel in the non-linear equation is our assumption only, based on the analogy with the leading and next-to-leading logarithmic calculations. This assumption is also motivated by the the fact that the dipole amplitude will still have the limiting value equal to unity in the saturation regime. In principle, the modification to the dipole kernel implies also the corrections to the triple Pomeron vertex, [66, 67]. This is an interesting problem which deserves further analysis but it is outside the scope of the present paper.

Recall first the leading-logarithmic analysis from [68] where the power tails in impact parameter space were shown to emerge just after the first step in the evolution in rapidity of the BFKL and BK equations. We take the initial condition for the amplitude to be exponentially decreasing in impact parameter

$$
N\left(y=\ln 1 / x=0, x_{01}\right) \equiv N_{01}^{(0)} \sim \exp \left(-\mu b_{01}\right),
$$

where the impact parameter is defined as $\underline{b}_{01}=\frac{1}{2}\left(\underline{x}_{0}+\underline{x}_{1}\right)$. Next, we perform one iteration of the dipole-BK equation with the LL kernel

$$
N_{01}(x)=N_{01}^{(0)}+\frac{\alpha_{s} N_{c}}{2 \pi^{2}} \int_{x}^{1} \frac{d z}{z} \int d^{2} \underline{x}_{2} \frac{x_{01}^{2}}{x_{02}^{2} x_{12}^{2}}\left[N_{02}+N_{12}-N_{01}-N_{02} N_{12}\right] .
$$

For a small dipole $x_{01}$ and at large value of the impact parameter $b_{01}$ the non-vanishing contribution to the amplitude comes from the configurations with large dipoles, i.e. such that one end is at $x_{0}$ and the other one is at $x_{2}$ located close to the center of the target where the field is strong. In that case $N_{01} \sim 0$ because of small dipole at large impact parameter and $N_{02} \sim N_{12} \sim 1$ and $x_{02} \sim x_{12} \sim b_{01}$. The r.h.s of the LL BK equation (97) is then

$$
\int d^{2} \underline{x}_{2} \frac{x_{01}^{2}}{x_{02}^{2} x_{12}^{2}}\left[N_{02}+N_{12}-N_{01}-N_{02} N_{12}\right] \simeq \frac{x_{01}^{2}}{b_{01}^{4}} \int_{\mathcal{R}} d^{2} \underline{x}_{2},
$$

where $\mathcal{R}$ is the integration region where approximately $x_{02} \sim b_{01}$. Therefore in the case of the LL equation the diffusion in the transverse space leads always to the power like tails in impact parameter even with the exponentially falling initial conditions. This leads to the violation of the Froissart bound [69] even in the presence of the saturation corrections, as was first pointed out in $70,71,72$.

The situation changes when the modified kernel (80) is considered. Here a similar analysis leads to

$$
\begin{aligned}
\int_{z_{0}-\delta z}^{z_{0}} \frac{d z}{z} \int d^{2} \underline{x}_{2} \bar{Q}_{01}^{2}\left[K_{1}^{2}\left(\bar{Q}_{01} x_{02}\right)+K_{1}^{2}\left(\bar{Q}_{01} x_{12}\right)-2 K_{1}\left(\bar{Q}_{01} x_{02}\right) K_{1}\left(\bar{Q}_{01} x_{12}\right) \frac{\underline{x}_{02} \cdot \underline{x}_{12}}{x_{02} x_{12}}\right] \times & \times\left[N_{02}+N_{12}-N_{01}-N_{02} N_{12}\right] \\
& \simeq \int_{z_{0}-\delta z}^{z_{0}} \frac{d z}{z} \int d^{2} \underline{x}_{2}\left[\theta\left(x_{01} / \sqrt{z} C-x_{02}\right) \frac{x_{01}^{2}}{x_{02}^{2} x_{12}^{2}}+\right. \\
+ & \left.\theta\left(x_{02}-x_{01} / \sqrt{z} C\right) \frac{\pi}{2} \frac{\sqrt{z} x_{01}}{x_{02}^{3}} \exp \left(-2 \frac{x_{02}}{x_{01}} \sqrt{z}\right)\right]\left[N_{02}+N_{12}-N_{01}-N_{02} N_{12}\right]
\end{aligned}
$$

where $C \sim 1$, and where we made approximations in the second term that $x_{01} \ll x_{12} \sim x_{02} \sim b_{01}$ which gives the dominant contribution. The first term is the short range contribution and the second one is the long range one. It is evident that the behavior of the scattering amplitude for small dipoles $x_{01}$ and at large values of the impact parameter $b_{01}$ is governed by

$$
N\left(x_{01}, b_{01}\right) \sim \exp \left(-2 \frac{b_{01}}{x_{01}} \sqrt{z}\right)
$$


Therefore there is an exponential but with the effective mass which becomes smaller as $\sqrt{z} / x_{01}$ decreases. One can actually see two limits in this behavior. We can take $b_{01}, x_{01}$ fixed and so by decreasing $z$, or increasing the energy, the exponential tails become power like. One can also take $b_{01}$ and $z$ fixed, and change the dipole size $x_{01}$. For small dipole sizes $x_{01} \ll b_{01} \sqrt{z}$ the tails are exponential, but for larger ones they become again power like. It is interesting to note that the largest effect of the modification is for the dipoles with small sizes, even though the cutoff inside the integral equation is acting on the large dipole sizes. This is result of the fact that the relevant parameter is $x_{02} / x_{01} \sqrt{z}$, i.e. is proportional to the ratio of the dipole sizes. The only way to eliminate the power-like tails is to put in (essentially by hand) the fixed mass term, which limits the range of the interactions [73], for example

$$
\bar{Q}_{01}^{2} \rightarrow \bar{Q}_{01}^{2}+m^{2}
$$

In this way one will get an amplitude which has exponential tails with fixed radius and this will lead to a behavior consistent with the Froissart bound [69] (modulo normalization).

\section{MHV SCATTERING AMPLITUDES FROM THE LIGHT-CONE WAVE FUNCTION}

\section{A. Parke-Taylor amplitudes}

In the previous sections we have analyzed the (tree level) wave function with arbitrary number of gluons in the light cone formulation. This corresponds to the initial state evolution, that precedes the scattering. In this section we take into account also the final state evolution and evaluate the production amplitude of $n$-gluons in 2 gluon scattering. The exact tree level amplitudes with an arbitrary number of the external on-shell gluons are known. These are Parke-Taylor amplitudes [8] (see [9] for a comprehensive review) and can be recast in the following form

$$
\mathcal{M}_{n}=\sum_{\{1, \ldots, n\}} \operatorname{tr}\left(t^{a_{1}} t^{a_{2}} \ldots t^{a_{n}}\right) m\left(p_{1}, \epsilon_{1} ; p_{2}, \epsilon_{2} ; \ldots ; p_{n}, \epsilon_{n}\right)
$$

where $a_{1}, a_{2}, \ldots, a_{n}, p_{1}, p_{2}, \ldots, p_{n}$ and $\epsilon_{1}, \epsilon_{2}, \ldots, \epsilon_{n}$ are the color indices, momenta and the helicities of the external $n$ gluons. Matrices $t^{a}$ are in the fundamental representation of the color $S U\left(N_{c}\right)$ group. The sum in (101) is over the $(n-1)$ ! non-cyclic permutations of the set $\{0,1, \ldots, n\}$. The kinematical parts of the amplitude denoted by

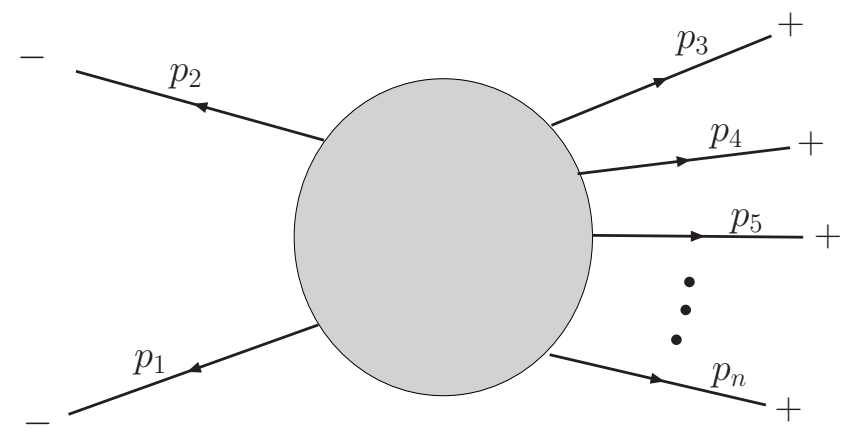

FIG. 4: The $n$-gluon production amplitude with $(-,-,+, \ldots,+)$ helicity configuration. All the gluons are taken to be outgoing.

$m(1,2, \ldots, n) \equiv m\left(p_{1}, \epsilon_{1} ; p_{2}, \epsilon_{2} ; \ldots ; p_{n}, \epsilon_{n}\right)$ are color independent and gauge invariant. These objects have a number of important properties

1. $m(1,2, \ldots, n)$ are invariant under cyclic permutations of the set $\{0,1, \ldots, n\}$.

2. $m(n, n-1, \ldots, 1)=(-1)^{n} m(1,2, \ldots, n)$ (reversal symmetry).

3. $m(1,2, \ldots, n)+m(2,1, \ldots, n)+m(2,3,1, \ldots, n)+\ldots+m(2,3, \ldots, n, 1)=0$ (dual Ward identity).

4. Incoherence to leading number of colors

$$
\sum_{\text {colors }}\left|\mathcal{M}_{n}\right|^{2}=N_{c}^{n-2}\left(N_{c}^{2}-1\right) \sum_{\{1, \ldots, n\}}\left\{|m(1,2, \ldots, n)|^{2}+\mathcal{O}\left(N_{c}^{2}\right)\right\} .
$$


It can be shown that the amplitudes where all the gluons have the same helicities, or only one is different from the others are vanishing (we assume that all the gluons are outgoing)

$$
m( \pm, \pm, \ldots, \pm)=m(\mp, \pm, \pm, \ldots, \pm)=0
$$

The non-vanishing amplitude for the configuration $(-,-,+, \ldots,+)$ depicted in Fig. 4 at the tree level is given by the formula

$$
m\left(1^{-}, 2^{-}, 3^{+}, \ldots, n^{+}\right)=i g^{n-2} \frac{\langle 12\rangle^{4}}{\langle 12\rangle\langle 23\rangle \ldots\langle n-2 n-1\rangle\langle n-1 n\rangle\langle n 1\rangle},
$$

where the spinor products are defined by Eqs. (17), (18).

The comparison of the BFKL amplitudes with the Parke-Taylor expressions was also performed some time ago in 74, 75] but only in the limit of the multi-Regge kinematics. Here, we shall re-derive the Parke-Taylor amplitudes within the color dipole picture in a much less restrictive kinematical limit. In order to compare the amplitudes obtained within the light cone perturbation theory formalism with the MHV amplitudes we need to consider the scattering process of the evolved wave function onto the target. We will simplify the problem by analyzing the case where the evolved projectile gluon scatters on a single target gluon which is separated from the virtual gluons in the projectile by a large rapidity interval. The exchange between the projectile and the target will be treated in the high energy limit. In this limit the interaction between the projectile and the target is mediated by an instantaneous part of the gluon propagator in the light cone gauge defined as

$$
D_{\text {inst }}^{\mu \nu}=\frac{\eta^{\mu} \eta^{\nu}}{\left(k^{+}\right)^{2}}
$$

see for example [5]. We restrict the kinematics of the exchange, but still, the internal structure of the projectile gluon field is accurately represented. In principle, the technique applied could be used to evaluate the scattering amplitudes without any kinematical restrictions, but an analysis of the completely general case would be much more complicated.
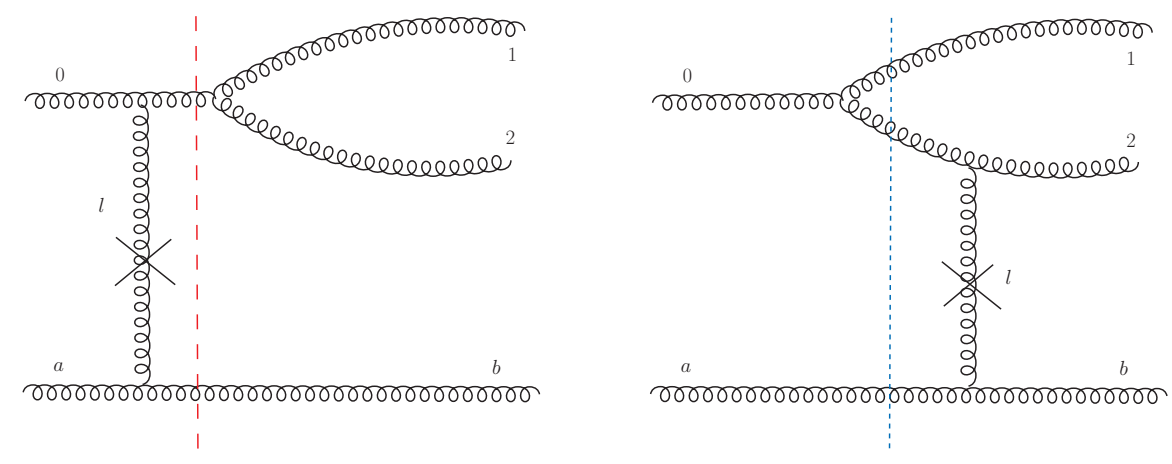

FIG. 5: Interaction of the wave function with 2 gluons with the target. The cross on the gluon line indicates that it is an instantaneous interaction. Vertical dashed lines denote the energy denominators; short dashes (blue lines), denominators in the initial radiation states; long dashed (red lines), denominators in the final state radiation.

In order to demonstrate the equivalence with the MHV amplitudes it is essential to include the final state radiation in the wave function, which occurs after the interaction with the target has taken place. For example, in the case of the $2 \rightarrow 3$ amplitude, we have to take into account graphs shown in Fig. 5. The gluon with the cross and labeled by the momentum $l$ is the Coulomb gluon, and the gluon with momentum $P$ is the target gluon. The latter one has a large $P^{-}$component. The dashed lines denote the energy denominators, which have to be taken before and after the interaction occurred. Similarly for the $2 \rightarrow 4$ amplitude we will need to take into account graphs shown in Fig. 6. In general for this kinematics the number of possible classes of diagrams is $n-3$ with $n-4$ energy denominators both in the initial and final state. Obviously within each of these classes one needs to sum over the different possibilities of the evolution of the wave function. For example in the case of graphs depicted in Fig. 6 there is a corresponding set of graphs where the last emitted gluon is coming from the splitting of the most upper gluon in the hadron wave function.

The dominance of the instantaneous (Coulomb) gluon exchange follows from the fact, that the other possible types of exchanges are suppressed w.r.t. the dominant exchange by large energy denominators. One can show easily that the relative suppression factor decrease with the total energy like $M^{2} / s$, where $M^{2}$ is the invariant mass of the system 

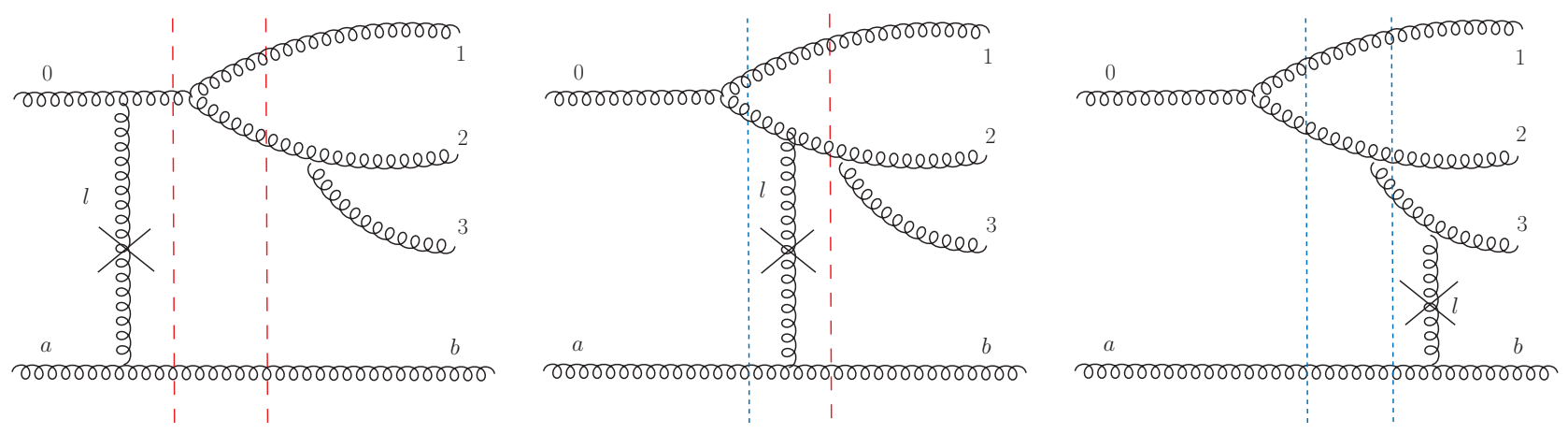

FIG. 6: Interaction of the wave function with 3 gluons with the target. The cross on the gluon line indicates that it is an instantaneous interaction. Vertical dashed lines denote the energy denominators; short dashes (blue lines), denominators in the initial radiation states; long dashed (red lines), denominators in the final state radiation.

produced by the projectile fragmentation. At fixed $M^{2}$, and for $s \rightarrow \infty$, the relative contributions of the non-Coulomb exchanges can be made arbitrarily small. Also, the contribution from scattering mediated by the four-gluon vertex is suppressed.

\section{B. Factorization of fragmentation tree amplitudes}

In constructing the full MHV amplitudes in the high energy limit we shall use the results for the initial state evolution supplemented by the analysis of fragmentation of the scattered states into on-shell final state gluons.

The key feature of the fragmentation amplitudes is the independence of fragmentation of the gluon trees originating from different parents. We consider a (typically disconnected) fragmentation tree with a topology $\Theta=\Theta_{1} \cup \ldots \cup \Theta_{m}$ where $\Theta_{1}, \ldots \Theta_{m}$ are topologies of the fragmentation trees of $m$ parent virtual gluons into $n$ on-shell gluons, so that the first parent gluon, denoted by $\left(12 \ldots n_{1}\right)$ fragments into gluons $\left(1,2, \ldots, n_{1}\right)$, the second parent gluon, $\left(n_{1}+1, n_{1}+2, \ldots, n_{1}\right)$ fragments into gluons $\left(n_{1}+1, n_{1}+2, \ldots, n_{2}\right)$, and so on. Then the amplitude of the fragmentation tree $\Theta$, denoted by $T_{\Theta}$ may be factorized into fragmentation amplitudes of the parent gluons, $T_{\Theta_{i}}$ in the following way, $T_{\Theta}\left[\left(1 \ldots n_{1}\right),\left(n_{1}+1 \ldots n_{2}\right), \ldots,\left(n_{m-1} \ldots n\right) \rightarrow 1,2, \ldots n\right]$,

$$
\begin{aligned}
& T_{\Theta}\left[\left(1 \ldots n_{1}\right),\left(n_{1}+1 \ldots n_{2}\right), \ldots,\left(n_{m-1} \ldots n\right) \rightarrow 1,2, \ldots n\right]=T_{\Theta_{1}}\left[\left(1 \ldots n_{1}\right) \rightarrow 1,2, \ldots n_{1}\right] \\
& \quad \times T_{\Theta_{2}}\left[\left(n_{1}+1 \ldots n_{2}\right) \rightarrow n_{1}+1, n_{1}+2, \ldots n_{2}\right] \times \ldots \times T_{\Theta_{m}}\left[\left(n_{m-1}+1 \ldots n\right) \rightarrow n_{m-1}, n_{m-1}+1, \ldots n\right],
\end{aligned}
$$

provided that we sum over all possible light-cone time orderings of the splittings within all connected trees, while preserving the topologies. This property is not obvious in the light-cone formulation, as for a given time ordering of the splittings, the variables related to different trees are mixed in the energy denominators, and the factorization property (103) does not hold. It only holds if the complete summation over all possible splitting orderings is performed. In fact, the factorization property of independent tree amplitudes is quite intuitive. Nevertheless we shall provide a formal proof of this property within the light-cone field theory framework.

In order to prove (103) we shall consider two sequences of splittings, $\left(a_{i}\right), i=0,1, \ldots, m$ and $\left(b_{j}\right), j=0,1, \ldots, n$, that occur in two topologically disconnected trees, $\Theta_{a}$ and $\Theta_{b}$, after the scattering. The internal topology of each tree is arbitrary, in particular each tree, $\Theta_{a}$ and $\Theta_{b}$, is not necessarily connected. The light-cone times of splittings $a_{i}$ and $b_{j}$ will be denoted by $\tau\left(a_{i}\right)$ and $\tau\left(b_{j}\right)$ respectively. In the computation of the fragmentation amplitude of the tree $\Theta=\Theta_{a} \cup \Theta_{b}$ one sums over all possible time-orderings of splittings. We shall denote the splitting ordering by $\mathcal{T}(\Theta)$. This summation may be decomposed into three nested summations: two independent external summations of the time orderings within each tree, $\Theta_{a}$ and $\Theta_{b}$, allowed by the topology, and the internal summation over all orderings of splittings between trees $\Theta_{a}$ and $\Theta_{b}$, with a fixed ordering within each tree,

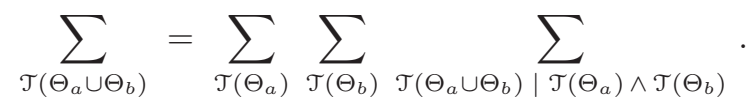

The two external summations are performed for each tree independently, so they preserve the factorization property (103). Therefore, it is enough to prove (103) for the arbitrary fixed splitting time orderings, $\mathcal{T}\left(\Theta_{a}\right)$ and $\mathcal{T}\left(\Theta_{b}\right)$. Thus, in what follows we fix the topologies $\Theta_{a}$ and $\Theta_{b}$ and the splitting orderings $\mathcal{T}\left(\Theta_{a}\right)$ and $\mathcal{T}\left(\Theta_{b}\right)$. It is convenient to label the splittings according to the time order, $\tau\left(a_{1}\right)<\tau\left(a_{2}\right)<\ldots<\tau\left(a_{m}\right)$ and $\tau\left(b_{1}\right)<\tau\left(b_{2}\right)<\ldots<\tau\left(b_{n}\right)$. 
When the topology of the tree $\Theta$ is fixed, then the contributions of splitting vertices to the amplitude does not depend on the time ordering of the splittings. Furthermore, this contributions factorize into a product of independent contribution from trees $\Theta_{a}$ and $\Theta_{b}$. This is not true only for the energy denominators that, in general, mix the variables of both trees. So, in order to complete the proof it is enough to show that the sum over relative splitting orderings, $\mathcal{T}\left(\Theta_{a} \cup \Theta_{b}\right) \mid \mathcal{T}\left(\Theta_{a}\right) \wedge \mathcal{T}\left(\Theta_{b}\right)$ of the products of the energy denominators of tree $\Theta$ factorizes between the trees $\Theta_{a}$ and $\Theta_{b}$. The energy denominators for tree $\Theta_{a}$, in the absence of tree $\Theta_{b}$, will be denoted by $A_{1}, A_{2}, \ldots, A_{m}$, where, in the final state we choose to attribute the denominator to the line before splitting. Analogously, the denominators for tree $\Theta_{b}$, in the absence of tree $\Theta_{a}$, will be denoted by $B_{1}, B_{2}, \ldots, B_{n}$. We shall prove the following factorization property for energy denominators:

$$
\sum_{\left(i_{p}, p=1, \ldots, n+m\right)} \prod_{p=1}^{n+m} \frac{1}{A_{i_{p}}+B_{p-i_{p}}}=\prod_{i=1}^{m} \frac{1}{A_{i}} \prod_{j=1}^{n} \frac{1}{B_{j}}
$$

where we used the additivity of the energy denominator of two evolving trees and introduced auxiliary symbols $A_{0}=B_{0}=0$. The series $\left(i_{p}, p=1, \ldots, n+m\right)$ represents the ordering of splittings between the chains in the following way: $i_{p}$ is the number of splittings of $\Theta_{a}$ corresponding to $p$ splittings of $\Theta_{a} \cup \Theta_{b}$ assuming the time ordering $\mathcal{T}\left(\Theta_{a} \cup \Theta_{b}\right) \mid \mathcal{T}\left(\Theta_{a}\right) \wedge \mathcal{T}\left(\Theta_{b}\right)$, and $p-i_{p}$ is the corresponding number of splittings of $\Theta_{b}$.

Equation (105) may be proven by double induction, with respect to $m$ and $n$. Let us first take $n=m=1$. Then we have:

$$
\frac{1}{A_{1}} \frac{1}{A_{1}+B_{1}}+\frac{1}{B_{1}} \frac{1}{A_{1}+B_{1}}=\frac{1}{A_{1} B_{1}}
$$

in accord with (105). Next, for $n=1$ and an arbitrary $m \geq 2$ we consider the l.h.s. of (105):

$$
\begin{aligned}
\sum_{q=1}^{m} \frac{1}{A_{1}+B_{1}} & \cdots \frac{1}{A_{q}+B_{1}} \frac{1}{A_{q}} \ldots \frac{1}{A_{m}}+\frac{1}{B_{1}} \frac{1}{A_{1}+B_{1}} \ldots \frac{1}{A_{m}+B_{1}} \\
= & \frac{1}{A_{1}+B_{1}}\left[\left(\sum_{q=2}^{m} \frac{1}{A_{q}} \ldots \frac{1}{A_{m}} \frac{1}{A_{2}+B_{1}} \cdots \frac{1}{A_{m}+B_{1}}\right)+\frac{1}{B_{1}} \frac{1}{A_{2}+B_{1}} \ldots \frac{1}{A_{m}+B_{1}}\right. \\
& \left.+\frac{1}{A_{1}} \ldots \frac{1}{A_{m}}\right]=\frac{1}{A_{1}+B_{1}}\left(\frac{1}{A_{2}} \ldots \frac{1}{A_{m}} \frac{1}{B_{1}}+\frac{1}{A_{1}} \ldots \frac{1}{A_{m-1}} \frac{1}{A_{m}}\right)=\frac{1}{A_{1}} \ldots \frac{1}{A_{m}} \frac{1}{B_{1}},
\end{aligned}
$$

where the first two terms in the square bracket in the middle line of (107) were evaluated using (105) for $m-1$. This proves (105) for $n=1$.

In the second step we use the mathematical induction w.r.t. $n$, for a fixed $m$. The first step is to split the sum, $\sum_{\left(i_{p}, p=1, \ldots, n+m\right)}$, into contributions $\Sigma_{1}, \Sigma_{2}, \ldots \Sigma_{m}, \Sigma_{m+1}$ in which the first splitting in the $b$-sequence, $b_{1}$, occurs before $a_{1}$, between $a_{1}$ and $a_{2}, \ldots$, between $a_{m-1}$ and $a_{m}$ and after $a_{m}$, respectively. In each of these contributions we sum over all the allowed orderings of the later splittings, $b_{2}, \ldots, b_{n}$, w.r.t. the $a_{i}$ splittings. Clearly, when $b_{1}$ occurs between $a_{q}$ and $a_{q+1}(1 \leq q \leq m-1)$, then all the later splittings, $b_{2}, \ldots, b_{n}$, must occur after the splitting $a_{q}$. It implies that in $\Sigma_{q}$ it is necessary to perform the complete summation over $b_{2}, \ldots, b_{n}$ orderings relative to the sequence $a_{1}, a_{2}, \ldots, a_{q}$. The splittings $\left\{a_{1}, a_{2}, \ldots, a_{q}, b_{1}, b_{2}, \ldots, b_{n-1}\right\}$ (with all possible orderings) are then followed by the (ordered) sequence of splittings: $\left(b_{n}, a_{q+1}, \ldots, a_{m}\right)$. The summation of the products of energy denominators over all orderings of the splittings $\left\{a_{1}, a_{2}, \ldots, a_{q}, b_{1}, b_{2}, \ldots, b_{n-1}\right\}$, preserving the ordering of $\left(a_{i}\right)$ and $\left(b_{j}\right)$ in $\Sigma_{q}$ may be evaluated by applying (105) for the number of $a$-splittings equal $n-1$ and the number of $b$-splittings equal $q$. The remaining denominators, corresponding to $\left(b_{n}, a_{q+1}, \ldots, a_{m}\right) \operatorname{read}, A_{q}+B_{1}, A_{q+1}+B_{1}, \ldots, A_{m-1}+B_{1}, A_{m}+B_{1}$, respectively. The final answer for $\Sigma_{q}$ reads,

$$
\Sigma_{q}=\left[\frac{1}{B_{2}} \ldots \frac{1}{B_{n}} \frac{1}{A_{q}} \ldots \frac{1}{A_{m}}\right] \frac{1}{A_{q}+B_{1}} \frac{1}{A_{q-1}+B_{1}} \cdots \frac{1}{A_{1}+B_{1}} .
$$


The l.h.s. of (105) is equal to sum over contributions $\Sigma_{q}$ :

$$
\begin{aligned}
\Sigma_{m}+\Sigma_{m-1} \ldots+\Sigma_{1}= & {\left[\frac{1}{B_{2}} \frac{1}{B_{2}} \ldots \frac{1}{B_{n}}\right] \frac{1}{B_{1}} \frac{1}{A_{1}+B_{1}} \frac{1}{A_{2}+B_{1}} \ldots \frac{1}{A_{m}+B_{1}} } \\
& +\left[\frac{1}{B_{2}} \frac{1}{B_{3}} \ldots \frac{1}{B_{n}} \frac{1}{A_{m}}\right] \frac{1}{A_{1}+B_{1}} \frac{1}{A_{2}+B_{1}} \ldots \frac{1}{A_{m}+B_{1}} \\
& +\ldots+\left[\frac{1}{B_{2}} \frac{1}{B_{3}} \ldots \frac{1}{B_{n}} \frac{1}{A_{m}} \frac{1}{A_{m-1}} \ldots \frac{1}{A_{2}}\right] \frac{1}{A_{2}+B_{1}} \frac{1}{A_{1}+B_{1}} \\
& +\left[\frac{1}{B_{2}} \frac{1}{B_{3}} \ldots \frac{1}{B_{n}} \frac{1}{A_{1}} \frac{1}{A_{2}} \ldots \frac{1}{A_{m}}\right] \frac{1}{A_{1}+B_{1}},
\end{aligned}
$$

where the contributions resummed using (105) are given in the square brackets. All the terms in the r.h.s. of (109) have a common prefactor $1 /\left(B_{2} \ldots B_{n}\right)$ that can be factored out. The summation of remaining factors in the l.h.s. of (109) is precisely the one evaluated in (107), and the result of this summation is given by $1 /\left(B_{1} A_{1} \ldots A_{m}\right)$. This completes the proof of the induction step in $n$, with fixed $m$. Taking into account the obvious symmetry between $m$ and $n$, it proves also the induction step in $m$, with fixed $n$. Therefore (105) holds for all $m$ and $n$. This, in turn, proves (103).

\section{Fragmentation of a single gluon}

For the comparison of the amplitudes obtained in the light cone perturbation theory with the known expressions for the MHV amplitudes it is necessary to compute both the initial and final state emissions. This is essential, as we need to take into a account graphs which include both type of processes as depicted in Figs. 5 and 6 We consider here the fragmentation of a single, off-shell gluon (labeled by $(12 \ldots n)$ ) into the final state of $n$ on-shell gluons, $1,2, \ldots n$. The $n$ final state gluons have transverse momenta $\underline{k}_{1}, \ldots, \underline{k}_{n}$ and the longitudinal momentum fractions $z_{1}, \ldots, z_{n}$. The initial gluon has transverse momentum $\underline{k}_{(1 \ldots n)}$ and the longitudinal fraction $z_{(1 \ldots n)}$ where we again used shortcut notation $\underline{k}_{(1 \ldots n)}=\sum_{j=1}^{n} \underline{k}_{j}$ and $z_{(12 \ldots n)}=\sum_{i=1}^{n} z_{i}$ (see Sec. III).

We will denote the fragmentation part of the amplitude for 1 to $\mathrm{n}$ gluons as $T[(12 \ldots n) \rightarrow 1,2, \ldots, n]$. Let us consider the $n=2$ case. Using the computational methods developed in Sec. III we can write the expression for the fragmentation in the following way

$$
T[(12) \rightarrow 1,2]=\frac{g}{\tilde{D}_{2}} \frac{v_{12}^{*}}{\sqrt{\xi_{12}}}=g \frac{v_{12}^{*}}{\xi_{12}^{3 / 2}\left|v_{12}\right|^{2}}=g \xi_{12}^{-3 / 2} \frac{1}{v_{12}} .
$$

To obtain the above expression we have used the formula for the 3-gluon vertex (6) and the definition of the complex variable $v_{12}$ (7). The energy denominator for the fragmentation $\tilde{D}_{2}$ was obtained from expression (12). The calculation for the fragmentation of one to three gluons reads

$$
\begin{array}{r}
T[(123) \rightarrow 1,2,3]=\frac{1}{\tilde{D}_{3}} g^{2}\left[\frac{v_{1(23)}^{*}}{\xi_{1(23)}^{1 / 2} \xi_{23}^{3 / 2} v_{23}}+\frac{v_{(12) 3}^{*}}{\xi_{(12) 3}^{1 / 2} \xi_{12}^{3 / 2} v_{12}}\right]= \\
=\frac{1}{\tilde{D}_{3}} g^{2}\left(\frac{z_{(123)}}{z_{1} z_{2} z_{3}}\right)^{3 / 2} \frac{v_{1(23)}^{*} \xi_{1(23)} v_{12}+v_{(12) 3}^{*} \xi_{(12) 3} v_{23}}{v_{12} v_{23}},
\end{array}
$$

where the energy denominator for this case

$$
\tilde{D}_{3}=\sum_{i=1}^{3} \frac{k_{i}^{2}}{z_{i}}-\frac{\left(k_{(123)}\right)^{2}}{z_{(123)}} .
$$

It is straightforward to verify that the numerator in the last line of (111) is equal to the denominator $\tilde{D}_{3}$. Note, that this is a case of $n=2$ of the generalization of the formula Eq. (42) derived in Sec. III, in the kinematics when the transverse momentum of the first particle in the fragmentation chain is not vanishing $k_{(123)} \neq 0$ and when $z_{(12 \ldots n)} \neq 1$. We also used the fact that

$$
\xi_{1(23)} \xi_{23}=\xi_{(12) 3} \xi_{12}=\frac{z_{(123)}}{z_{1} z_{2} z_{3}}
$$


Based on these two examples for $n=2$ and $n=3$ we postulate a general formula, that generalizes computations carried out explicitly for $n \leq 4$. The conjectured amplitude of fragmentation depicted in Fig. (7) reads

$$
T[(12 \ldots n) \rightarrow 1,2, \ldots, n]=g^{n-1}\left(\frac{z_{(12 \ldots n)}}{z_{1} z_{2} \ldots z_{n}}\right)^{3 / 2} \frac{1}{v_{12} v_{23} \ldots v_{n-1 n}} .
$$

It is interesting to note that the form of the above amplitude for the fragmentation is dual to that of the gluon wave function with $n$-components. Namely, upon the replacement of the gluon light-cone velocities $v_{i j}$ in (113) by the gluon transverse positions $r_{i j}$, we obtain the formula (52) for the on-shell gluon wave function in the coordinate space (modulo the overall factors with $z_{i}$ fractions).

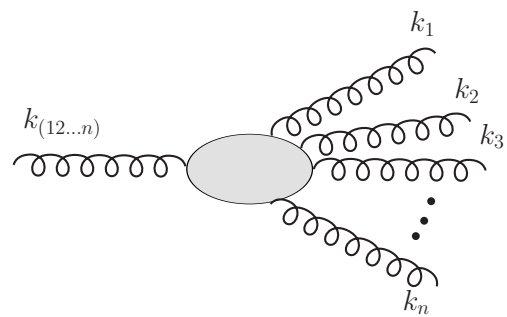

FIG. 7: Pictorial representation of the fragmentation amplitude $T[(12 \ldots n) \rightarrow 1,2, \ldots, n]$ for the single off-shell initial gluon.

In order to prove the above formula (113) we will need first to show that the relation (112) is valid for arbitrary values of $n$. The generalization of Eq. (42) can be easily shown by noting that in this case we have

$$
v_{(1 \ldots i)(i+1 \ldots n)} \xi_{(1 \ldots i)(i+1 \ldots n)}=\sum_{j=1}^{i} k_{j}-\frac{z_{(1 \ldots i)}}{z_{(1 \ldots n)}} \sum_{j=1}^{n} k_{j}=k_{(1 \ldots i)}-\frac{z_{(1 \ldots i)}}{z_{(1 \ldots n)}} k_{(1 \ldots n)}
$$

which is also a generalization of relation (43) for the case when $\sum_{j=1}^{n} k_{j} \neq 0$. The analogous proof as in (42) can be shown to proceed as follows

$$
\begin{aligned}
& \sum_{i=1}^{n} v_{i i+1} \xi_{(1 \ldots i)(i+1 \ldots n+1)} v_{(1 \ldots i)(i+1 \ldots n+1)}=\sum_{i=1}^{n} v_{i i+1}\left(\sum_{j=1}^{i} k_{j}-\frac{z_{(1 \ldots i)}}{z_{(1 \ldots n+1)}} \sum_{j=1}^{n+1} k_{j}\right)= \\
& =\sum_{i=1}^{n}\left(\frac{k_{i}}{z_{i}}-\frac{k_{i+1}}{z_{i+1}}\right)\left(\sum_{j=1}^{i} k_{j}-\frac{z_{(1 \ldots i)}}{z_{(1 \ldots n+1)}} \sum_{j=1}^{n+1} k_{j}\right) \\
& =\sum_{i=1}^{n} \frac{k_{i}^{2}}{z_{i}}+\frac{k_{n+1}^{2}}{z_{n+1}}-\frac{k_{n+1}}{z_{n+1}} \sum_{j=1}^{n+1} k_{j}-\frac{\sum_{j=1}^{n+1} k_{j}}{\sum_{j=1}^{n+1} z_{j}}\left[\sum_{i=1}^{n} \frac{k_{i}}{z_{i}} z_{i}+\sum_{i=1}^{n} \frac{k_{i}}{z_{i}} \sum_{j=1}^{i-1} z_{j}-\frac{k_{n+1}}{z_{n+1}} \sum_{j=1}^{n} z_{j}-\sum_{i=1}^{n-1} \frac{k_{i+1}}{z_{i+1}} \sum_{j=1}^{i} z_{j}\right] .
\end{aligned}
$$

Again, the second and fourth terms in the [...] cancel and we are left with

$$
\begin{array}{r}
\sum_{i=1}^{n+1} \frac{k_{i}^{2}}{z_{i}}-\frac{k_{n+1}}{z_{n+1}} \sum_{j=1}^{n+1} k_{j}-\frac{\sum_{j=1}^{n+1} k_{j}}{\sum_{j=1}^{n+1} z_{j}}\left[\sum_{j=1}^{n} k_{j}-\frac{k_{n+1}}{z_{n+1}}\left(\sum_{j=1}^{n+1} z_{j}-z_{n+1}\right)\right]= \\
=\sum_{i=1}^{n+1} \frac{k_{i}^{2}}{z_{i}}-\frac{\left(\sum_{j=1}^{n+1} k_{j}\right)^{2}}{\sum_{j=1}^{n+1} z_{j}}=\sum_{i=1}^{n+1} \frac{k_{i}^{2}}{z_{i}}-\frac{\left(k_{(1 \ldots n+1)}\right)^{2}}{z_{(1 \ldots n+1)}}=\tilde{D}_{n+1} .
\end{array}
$$

The proof of the conjectured amplitude (113) can now be performed by mathematical induction, using the factorization property (103) and the relation (116). Let us take the fragmentation into $n+1$ gluons denoted by $T[(1,2, \ldots, n+1) \rightarrow 1,2, \ldots, n+1]$ and represent it by lower fragmentation factors $T[(1 \ldots i) \rightarrow 1, \ldots, i]$ and 
$T[(i+1 \ldots n+1) \rightarrow i+1, \ldots, n+1]$ by separating out the first splitting (in all possible realizations). We obtain

$$
\begin{aligned}
T[(12 \ldots n+1) \rightarrow 1,2, \ldots, n+1]=\frac{g}{\tilde{D}_{n+1}} & \sum_{i=1}^{n}\left\{\frac{v_{(1 \ldots i)(i+1 \ldots n+1)}^{*}}{\sqrt{\xi_{(1 \ldots i)(i+1 \ldots n+1)}}}\right. \\
\times & T[(1 \ldots i) \rightarrow 1, \ldots, i] T[(i+1 \ldots n+1) \rightarrow i+1, \ldots, n+1]\}
\end{aligned}
$$

This expression is the final state analog of formula (15) for the iteration of the wave function and it is schematically depicted in Fig. 8. This may be rewritten using formula (113) for lower number of gluons:

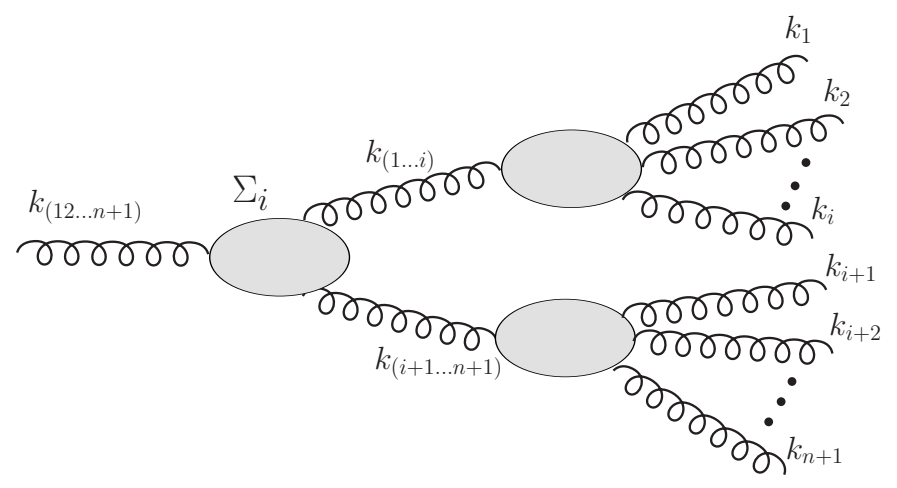

FIG. 8: Pictorial representation of one step in the fragmentation, Eq. (117).

$$
\begin{array}{r}
T[(12 \ldots n+1) \rightarrow 1,2, \ldots, n+1]=g^{n} \frac{1}{\tilde{D}_{n+1}} \sum_{i=1}^{n}\left(\frac{z_{(1 \ldots i)} z_{(i+1 \ldots n+1)}}{z_{1} \ldots z_{i} z_{i+1} \ldots z_{n+1}}\right)^{3 / 2} \frac{1}{\xi_{(1 \ldots i)(i+1 \ldots n+1)}^{3 / 2}} \\
\times \frac{\xi_{(1 \ldots i)(i+1 \ldots n+1) v_{(1 \ldots i)(i+1 \ldots n+1)}^{*}}}{\left(v_{12} \ldots v_{i-1 i}\right)\left(v_{i+1 i+2} \ldots v_{n n+1}\right)}
\end{array}
$$

The expression

$$
\left(\frac{z_{(1 \ldots i)} z_{(i+1 \ldots n+1)}}{z_{1} \ldots z_{i} z_{i+1} \ldots z_{n+1}}\right)^{3 / 2} \frac{1}{\xi_{(1 \ldots i)(i+1 \ldots n+1)}^{3 / 2}}=\left(\frac{z_{(12 \ldots n+1)}}{z_{1} z_{2} \ldots z_{n+1}}\right)^{3 / 2}
$$

is independent of $i$ and can be factored out in front of the sum. Taking the common denominator we finally arrive at

$$
\begin{gathered}
T[(12 \ldots n+1) \rightarrow 1,2, \ldots, n+1]=\left(\frac{z_{(12 \ldots n+1)}}{z_{1} z_{2} \ldots z_{n+1}}\right)^{3 / 2} \frac{g^{n}}{\tilde{D}_{n+1}} \frac{\sum_{i=1}^{n} \xi_{(1 \ldots i)(i+1 \ldots n+1)} v_{(1 \ldots i)(i+1 \ldots n+1)}^{*} v_{i i+1}}{v_{12} v_{23} \ldots v_{n n+1}}= \\
g^{n}\left(\frac{z_{(12 \ldots n+1)}}{z_{1} z_{2} \ldots z_{n+1}}\right)^{3 / 2} \frac{1}{v_{12} v_{23} \ldots v_{n n+1}}, \quad(11
\end{gathered}
$$

where to get the last line we used the proof in Eqs. (115 116) that the sum in the numerator in the first line is equal to the energy denominator $\tilde{D}_{n+1}$. The above expression is identical to (113) for $n+1$ which completes the proof.

\section{MHV amplitudes from the LCPT}

It is interesting to see how the MHV amplitudes emerge in the LCPT framework. We have derived the exact expressions for the light cone wave functions in Sec. III and the fragmentation amplitudes of the virtual gluon in Sec. VC. In this section we compute the $2 \rightarrow n+1$ gluon scattering amplitude in the limit when a projectile gluon produces $n$ gluons off a single gluon target, and the produced states is far in rapidity from the target gluon. The situation is depicted schematically in Fig. 9. The result will be shown to reproduce the known analytic form of the 


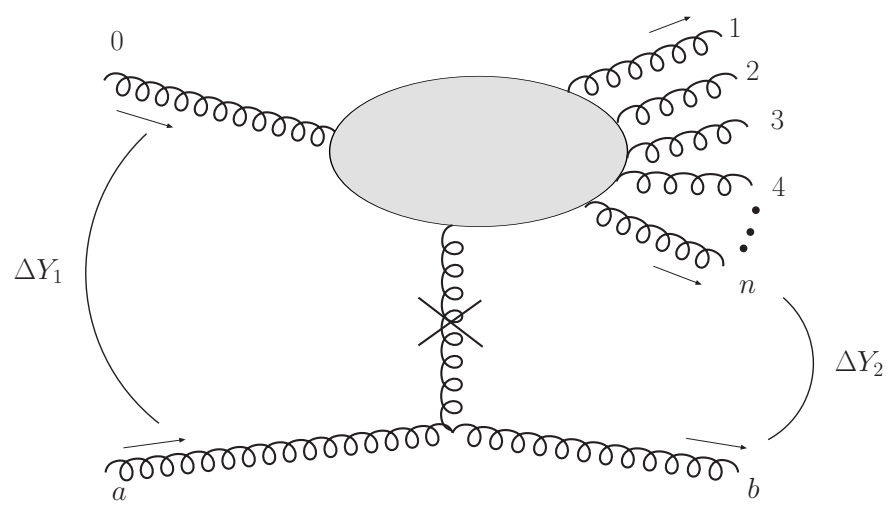

FIG. 9: The $2 \rightarrow n+1$ on-shell gluon amplitude in the high energy limit. The gluon 0 dissociates into the gluon cascade (indicated by a blob) which interacts via high energy gluon (with a cross) with the gluon $a \rightarrow b$. The large rapidity difference $\Delta Y_{1} \sim \Delta Y_{2}$ between the light cone cascade and the lower gluon is taken. The arrows indicate the momentum flow: the gluons $0, a$ are incoming and $1, \ldots, n, b$ are outgoing. All the gluons have + helicity and it is conserved.

MHV amplitudes in the adopted kinematic limit. In fact, due to this kinematic limitation we do not provide here the exact derivation of the MHV amplitudes in the general case. Note, however, that the high energy approximation is made only for the coupling of the incoming and outgoing target gluon, and no other approximations are made. Therefore, the comparison of our results to the MHV amplitudes provides a thorough cross check of our approach and the calculations.

We label the gluons in the way shown in Fig. 9. The incoming gluon labeled 0, develops into a cascade of gluons $1, \ldots, n$ and scatters on a target gluon $a \rightarrow b$. For the high energy case the dominant contributions are given by the instantaneous exchange of the Coulomb gluon. The exchanged gluon can be attached to any gluon in the cascade. Therefore we include both initial and final state emissions. However, since we are considering color ordered amplitudes it will be sufficient to take into account the attachment to the gluons in the form of the diagrams shown in Figs. 2 and 5. The gluons 0 and $a$ are incoming with helicities + , and all gluons through the whole cascade down to the final state, carry the positive helicity.

In the simplest case when the upper part of the diagram is just single gluon $0 \rightarrow 1$ we have only $2 \rightarrow 2$ scattering and the helicity is conserved. The MHV amplitude for $2 \rightarrow 2$ scattering (102) in this case

$$
|M(a, 0 \rightarrow 1, b)|=g^{2}\left|\frac{\langle a 0\rangle^{4}}{\langle a 0\rangle\langle 01\rangle\langle 1 b\rangle\langle b a\rangle}\right|=g^{2} \frac{s}{|t|},
$$

where we have used the fact that the spinor products can be regarded as complex square roots of the Mandelstam invariants, see formulae (19), and in this case they are equal to

$$
|\langle a 0\rangle|=|\langle 1 b\rangle|=\sqrt{s}, \quad|\langle 01\rangle|=|\langle b a\rangle|=\sqrt{|t|} .
$$

The explicit computation of the Coulomb gluon exchange in the LCPT in the high energy limit gives

$$
|\widetilde{M}(a, 0 \rightarrow 1, b)|=\frac{g^{2}}{\sqrt{z_{a} z_{b} z_{0} z_{1}}} \frac{s}{|t|},
$$

Obviously results (120) and (121) agree up to the conventional factor $1 / \sqrt{z_{a} z_{b} z_{0} z_{1}}$, used in the LCPT amplitudes [5, 6].

In what follows, we shall factorize the amplitude of the gluon exchange, $\sim g^{2} s /|t|$, from the amplitude of the projectile gluon evolution down to the $n$-gluon final state, $\tilde{\Psi}_{n}$. Thus, we write:

$$
\widetilde{M}(a, 0 \rightarrow b, 1,2, \ldots n)=\frac{1}{\sqrt{z_{a} z_{b}}} g^{2} \frac{s}{|t|} \tilde{\Psi}_{n}(1,2, \ldots n),
$$

where the prefactor $1 / \sqrt{z_{a} z_{b}}$ is, again, a result of the convention adopted in the LCPT, and as usual the momentum conservation $\delta$-functions represented by $\Delta^{(n+1)}$ (recall that this factor is implicit in the wave functions) are factored out from the amplitude. In general, we have,

$$
M(a, 0 \rightarrow b, 1,2, \ldots n)=\sqrt{z_{a} z_{b} z_{0} z_{1} \ldots z_{n}} \widetilde{M}(a, 0 \rightarrow b, 1,2, \ldots n)
$$


Let us now consider the $2 \rightarrow 3$ process depicted in Fig. 2 Using the light cone framework we can rewrite the upper part of both graphs in the following way

$$
\tilde{\Psi}_{2}(1,2)=\Psi_{1}\left(12^{\prime}\right) T[(12) \rightarrow 1,2]+\Psi_{2}\left(1,2^{\prime}\right),
$$

where the first term on the right hand side of the above equation (fragmentation in the final state) corresponds to the upper portion of left graph in Fig. 2 and the second term (the initial wave function) to the analogous part in the graph on the right hand side. To be more precise we represent Eq. (124) graphically in Fig. 10, Note, that the prefactor $z_{2}$, related to the Coulomb gluon coupling to gluons 2 and $2^{\prime}$, cancels against the factors of $1 / \sqrt{z_{2}}$, coming from the vertices connected by gluon $2^{\prime}$. The notation $2^{\prime}$ indicates that we need to take into account the transverse

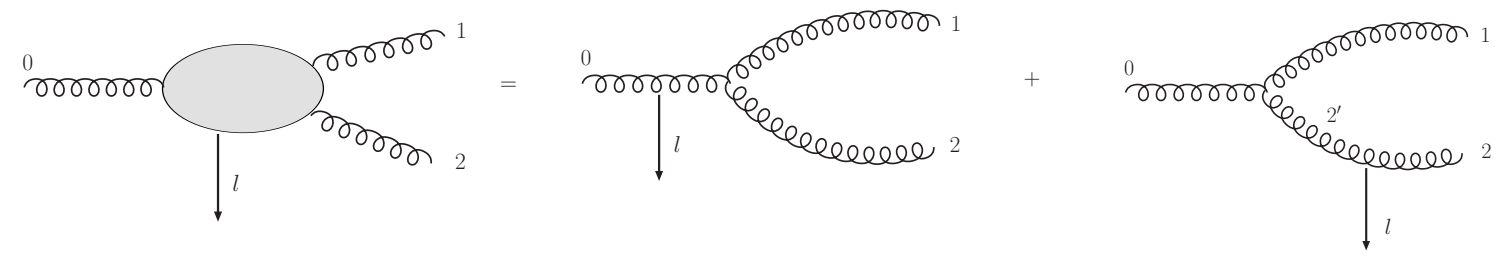

FIG. 10: Pictorial representation of Eq. (124). The down-pointing arrows indicate the momentum transfer caused by the exchange of the $t$ channel gluon.
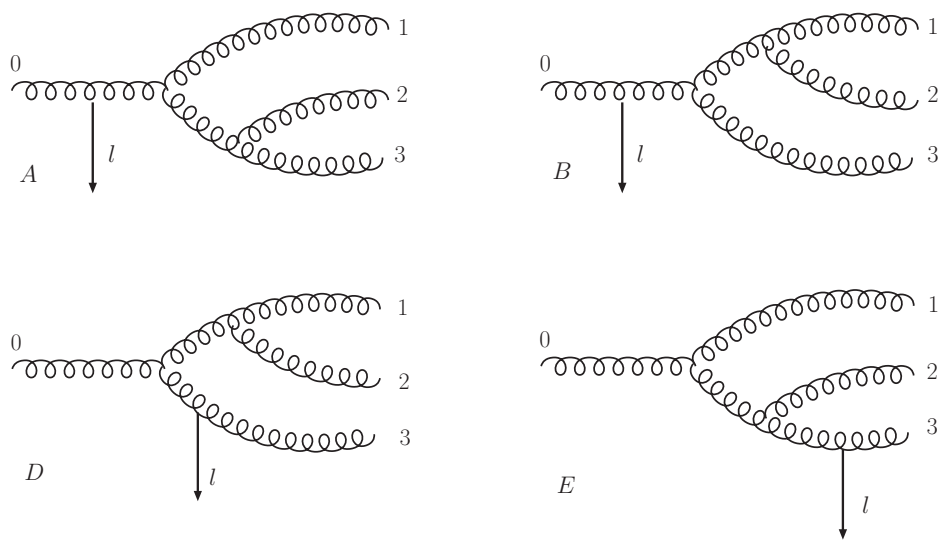

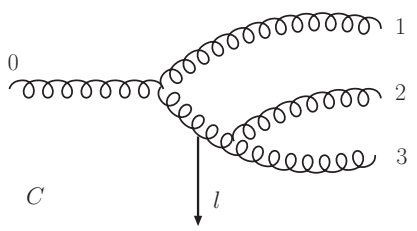

FIG. 11: Diagrams contributing to the $2 \rightarrow 4$ amplitude. The down-pointing arrows indicate the momentum transfer caused by the exchange of the $t$ channel gluon.

momentum carried by the exchanged gluon (the longitudinal fraction of the gluon momentum is neglected). It is equal to $l=-k_{(12)}$. For the process depicted in the last graph in Fig. 10 we have $k_{2^{\prime}}=k_{2}+k_{(12)}=-k_{1}$. Putting in the explicit expressions for the wave function $\Psi_{2}\left(1,2^{\prime}\right)$ Eq. (38) and $T[(12) \rightarrow 1,2]$ Eq. (113) we obtain

$$
\tilde{\Psi}_{2}(1,2)=g\left(\frac{1}{\left(z_{1} z_{2}\right)^{3 / 2}} \frac{1}{v_{12}}-\frac{1}{\left(z_{1} z_{2}\right)^{3 / 2}} \frac{1}{v_{12^{\prime}}}\right)=\frac{g}{\left(z_{1} z_{2}\right)^{3 / 2}} \frac{v_{12^{\prime}}-v_{12}}{v_{12} v_{12^{\prime}}}=\frac{g}{\left(z_{1} z_{2}\right)^{3 / 2}} \frac{z_{1} k_{(12)}}{k_{1} v_{12}} .
$$

For the case of the $2 \rightarrow 4$ process we have the following contributions

$$
\tilde{\Psi}_{3}(1,2,3)=\Psi_{1}\left(123^{\prime}\right) T[(123) \rightarrow 1,2,3]+\Psi_{2}\left(1,(23)^{\prime}\right) T[(23) \rightarrow 2,3]+\Psi_{2}\left(12,3^{\prime}\right) T[(12) \rightarrow 1,2]+\Psi_{3}\left(1,2,3^{\prime}\right) .
$$

The first term on the right hand side corresponds to the sum of the diagrams A and B in Fig. [11 the second and the third terms correspond to the diagrams $\mathrm{C}$ and $\mathrm{D}$, and the last term is the sum of diagrams $\mathrm{E}$ and $\mathrm{F}$. Again, the explicit calculation using (38) and (113) yields

$$
\begin{aligned}
\tilde{\Psi}_{3}(1,2,3)= & g^{2}\left[\frac{1}{\left(z_{1} z_{2} z_{3}\right)^{3 / 2}} \frac{1}{v_{12} v_{23}}-\frac{1}{\xi_{1(23)}^{3 / 2}} \frac{1}{v_{1(23)^{\prime}}}\left(\frac{1}{\xi_{23}}\right)^{3 / 2} \frac{1}{v_{23}}\right. \\
& \left.-\frac{1}{\xi_{(12) 3}^{3 / 2}} \frac{1}{v_{(12) 3^{\prime}}}\left(\frac{1}{\xi_{12}}\right)^{3 / 2} \frac{1}{v_{12}}+\frac{1}{\sqrt{z_{1} z_{2} z_{3}}} \frac{1}{\xi_{1(23)} \xi_{(12) 3}} \frac{1}{v_{(12) 3^{\prime}} v_{1(23)^{\prime}}}\right]=\frac{g^{2}}{\left(z_{1} z_{2} z_{3}\right)^{3 / 2}} \frac{k_{(123)} z_{1}}{k_{1} v_{12} v_{23}} .
\end{aligned}
$$


In deriving (127) we used the following relations

$$
v_{1(23)^{\prime}}=k_{1} \frac{1}{z_{1}\left(z_{2}+z_{3}\right)}=\frac{k_{1}}{\xi_{1(23)}}, \quad v_{(12) 3^{\prime}}=k_{(12)} \frac{1}{z_{3}\left(z_{1}+z_{2}\right)}=\frac{k_{(12)}}{\xi_{(12) 3}},
$$

as well as $\xi_{1(23)} \xi_{23}=\xi_{(12) 3} \xi_{12}=z_{1} z_{2} z_{3}$. The case for $2 \rightarrow 5$ proceeds in analogy. The corresponding expression for $\tilde{\Psi}_{4}$ can be found by taking the following sum

$$
\begin{aligned}
& \tilde{\Psi}_{4}=\Psi_{4}\left(1,2,3,4^{\prime}\right)+ \\
& \Psi_{3}\left(1,2,(34)^{\prime}\right) T[(34) \rightarrow 3,4]+\Psi_{3}\left(1,23,4^{\prime}\right) T[(23) \rightarrow 2,3]+\Psi_{3}\left(12,3,4^{\prime}\right) T[(12) \rightarrow 1,2]+ \\
& \Psi_{2}\left(1,(234)^{\prime}\right) T[(234) \rightarrow 2,3,4]+\Psi_{2}\left(1,(234)^{\prime}\right) T[(234) \rightarrow 2,3,4]+ \\
& \Psi_{2}\left((12),(34)^{\prime}\right) T[(12) \rightarrow 1,2] T[(34) \rightarrow 3,4]+\Psi_{1}\left(1234^{\prime}\right) T[(1234) \rightarrow 1,2,3,4],
\end{aligned}
$$

where we have considered all possible attachments of the Coulomb gluon. The explicit calculations are straightforward but lengthy, with the result being

$$
\tilde{\Psi}_{4}=g^{3} \frac{1}{\left(z_{1} z_{2} z_{3} z_{4}\right)^{3 / 2}} \frac{z_{1} k_{(1234)}}{k_{1} v_{12} v_{23} v_{34}} .
$$

The expression for the general $n$ can be found from the following formula

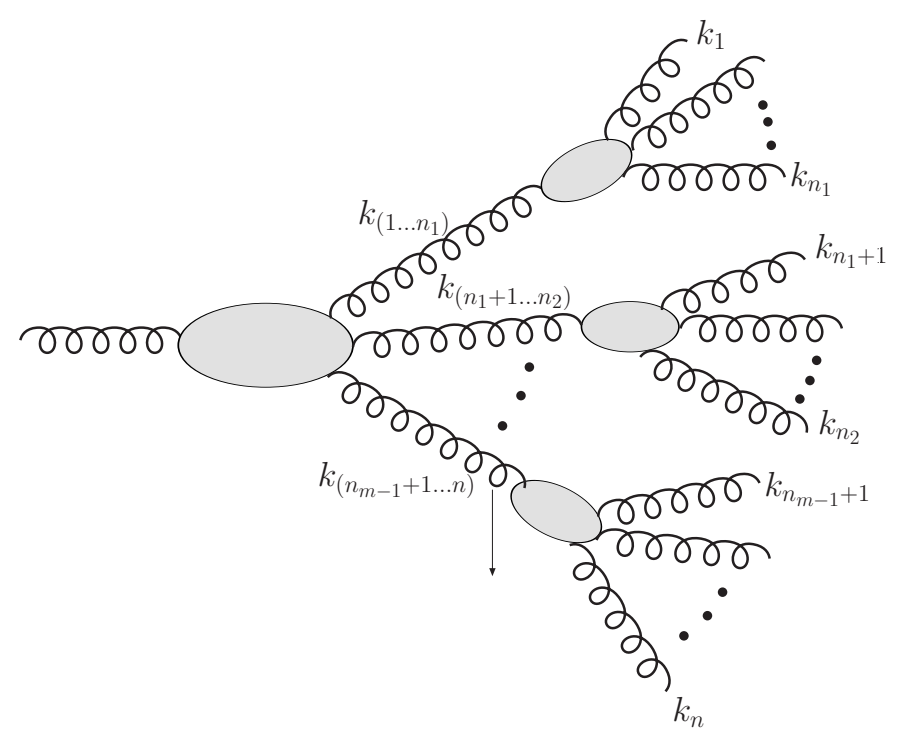

FIG. 12: Schematic representation of formula (130). The down-pointing arrow indicates the momentum transfer due to the exchange of the Coulomb gluon. The part of the graph to the left of this arrow (before the scattering) is the wave function $\Psi_{m}$ and the part of the graph to the right are the fragmentation amplitudes $T$, see Eq. (130).

$$
\begin{aligned}
& \tilde{\Psi}_{n}(1,2, \ldots, n)=\sum_{m=1}^{n} \sum_{\left(1 \leq n_{1}<n_{2}<\ldots<n_{m-1} \leq n\right)} \Psi_{m}\left(\left(1 \ldots n_{1}\right)\left(n_{1}+1 \ldots n_{2}\right) \ldots\left(n_{m-1}+1 \ldots n\right)\right) \\
& \times T\left[\left(1 \ldots n_{1}\right) \rightarrow 1, \ldots, n_{1}\right] T\left[\left(n_{1}+1 \ldots n_{2}\right) \rightarrow n_{1}+1, \ldots, n_{2}\right] \ldots T\left[\left(n_{m-1}+1 \ldots n\right) \rightarrow n_{m-1}+1, \ldots, n\right] .
\end{aligned}
$$

This formula, depicted in Fig. 12] is obtained by taking into account all possible attachments of the exchanged gluon in the cascade. The additional assumption is the factorization of fragmentation of virtual gluon (103). For the complete proof one has to use the explicit expressions for the fragmentation amplitude $T$ given by (113) and the initial state wave function $\Psi_{n}$ given by (38). The general proof is quite lengthy therefore we give it in the appendix A. The final result is the following general form for the $\tilde{\Psi}_{n}$ for an arbitrary number of emitted gluons

$$
\tilde{\Psi}_{n}(1,2, \ldots n)=g^{n-1} \frac{k_{(1 \ldots n)}}{k_{1} / z_{1}} \frac{1}{\sqrt{z_{1} z_{2} \ldots z_{n}}} \frac{1}{z_{1} z_{2} \ldots z_{n}} \frac{1}{v_{12} v_{23} \ldots v_{n-1 n}} .
$$




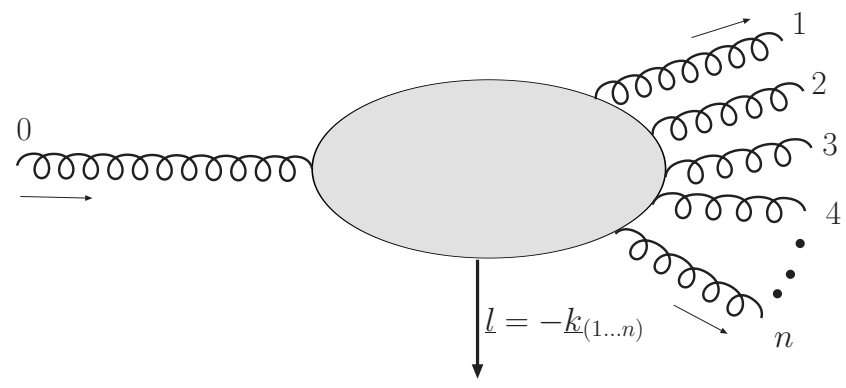

FIG. 13: Pictorial representation of $\tilde{\Psi}_{n}$. The down-pointing arrow indicates the momentum transfer caused by the exchange of the $t$ channel gluon. The sum over all possible attachments to the gluons in the cascade is performed.

We see that it is a generalization of the case for $n=2,3,4$ given in (125), (127) and (129). Note that, $v_{01}=-\frac{k_{1}}{z_{1}}$ (as we have chosen the transverse momentum of particle 0 to vanish $\left.\underline{k}_{0}=0\right)$. This object is graphically represented in Fig. 13. In order to facilitate the comparison with the MHV amplitude we use the relation $\langle i i+1\rangle=\sqrt{z_{i} z_{i+1}} v_{i i+1}$ to get

$$
\tilde{\Psi}_{n}(1,2, \ldots n)=g^{n-1} \frac{1}{\sqrt{z_{1} z_{2} \ldots z_{n}}} \frac{1}{\sqrt{z_{n}}} \frac{k_{(1 \ldots n)}}{\langle 01\rangle\langle 12\rangle\langle 23\rangle \ldots\langle n-1 n\rangle} .
$$

We also need to include the Coulomb gluon exchange between gluon $a \rightarrow b$ and the gluons in the cascade. Let us rewrite the $s /|t|$ part of the prefactor in (122),

$$
\frac{s}{|t|}=\sqrt{z_{n}}\left|\frac{\langle a 0\rangle^{4}}{\langle a 0\rangle\langle n b\rangle\langle b a\rangle k_{(1 \ldots n)}}\right|+\mathcal{O}\left(s^{0}\right) .
$$

Collecting together (122), (123), (132), and (133), we obtain (up to a complex phase factor) the amplitude for $2 \rightarrow n+1$ scattering

$$
M(0 ; a \rightarrow 1, \ldots, n ; b) \simeq g^{n+1} \frac{\langle a 0\rangle^{4}}{\langle a 0\rangle\langle 01\rangle\langle 12\rangle\langle n-1 n\rangle\langle n b\rangle\langle b a\rangle},
$$

which is equivalent to the MHV amplitude (102). The approximation sign " $\simeq$ " is used because the exchange between the gluon cascade and gluon $a$ was computed in the high energy limit.

\section{Summary}

In this paper we have analyzed multi-gluon cascades keeping the exact representation of the gluon kinematics using the light cone perturbation theory. For the choice of gluon helicities corresponding to the MHV amplitudes, we considered the components of the incoming gluon light cone wave function with an arbitrary number of virtual gluons. We found a hierarchy of recurrence relation between the multi-gluon components that holds at the tree level. The hierarchy was solved exactly in the case of the on-shell incoming gluon. A compact exact form of the real gluon wave function is presented. Interestingly enough, the natural variables which appear in the computation are closely related to the spinor products which appear in the computation of the maximally helicity violating amplitudes.

We also improved the color dipole evolution equation at small $x$ by relaxing partially the soft gluon approximation. Thus, we included into analysis an entanglement between the longitudinal and transverse degrees of freedom, as it follows from the form of the energy denominators of the light cone perturbation theory. We obtained a modified kernel of the color dipole evolution in which the emission of large dipoles is exponentially suppressed above the size dependent on the longitudinal momentum fraction of the softer emission in the dipole / gluon splitting. The new kernel contains modified Bessel function of the second kind and it is no longer conformally invariant in two transverse dimensions. It also leads to a much slower diffusion in transverse space than the original LL dipole kernel.

Furthermore, we showed that the modified dipole evolution kernel gives at the NLL accuracy the most singular pieces at the collinear limit of the exact NLL BFKL kernel, related to double logarithmic terms in the collinear limit. Notably, these are the terms of the NLL BFKL kernel which violate the conformal invariance in the two transverse dimensions both in the case of the $\mathcal{N}=4$ SYM theory and in the case of QCD case. This supports a conjecture that these terms are related rather to universal kinematical effects than to dynamical details of the theory. 
We have also investigated the scattering of the gluon, described by the light cone wave function on a target gluon and the final state emissions of gluons. We showed explicitly within LCPT that fragmentation amplitudes of different virtual gluons emerging from the scattering into real gluons are independent, despite an apparent entanglement. The fragmentation amplitude has been computed exactly of a single virtual gluon with the positive helicity into an arbitrary number of real gluons with positive helicities. Interestingly, we found a duality between the initial state wave function of the real gluon and the fragmentation amplitude of the virtual gluon. This result may hint on a deeper duality between the initial and final state evolution in the gluon cascade.

Finally, we have constructed the $2 \rightarrow n+1$ gluon amplitudes in the MHV helicity configuration by scattering the wave function on the target gluon, followed by the fragmentation of the scattered state. We chose the kinematical situation in which the projectile gluon produces $n$ gluons in arbitrarily large rapidity separation from the target gluon. In this limit we reproduced the Parke-Taylor MHV amplitudes.

\section{Acknowledgments}

We especially thank Ted Rogers for discussions. We also thank Jochen Bartels, Francois Gelis, Al Mueller, Gregory Soyez and Raju Venugopalan for comments and discussions. This work was supported by the Polish Ministry of Education grant No. N202 249235. L. M. gratefully acknowledges the support of the DFG grant No. SFB 676.

\section{Appendix A}

We provide here the proof of the explicit expression for the upper portion of the scattering amplitudes (131) which will be done via recursive induction. Let us begin with the formula (130) for the function $\tilde{\Psi}_{n}$ introduced in Sec. $\nabla$

$$
\begin{aligned}
\tilde{\Psi}_{n}(1,2, \ldots, n) & =\sum_{m=1}^{n} \sum_{\left(1 \leq n_{1}<n_{2}<\ldots<n_{m-1} \leq n\right)} \Psi_{m}\left(\left(1 \ldots n_{1}\right)\left(n_{1}+1 \ldots n_{2}\right) \ldots\left(n_{m-1}+1 \ldots n\right)\right) \\
\times T\left[\left(1 \ldots n_{1}\right)\right. & \left.\rightarrow 1, \ldots, n_{1}\right] T\left[\left(n_{1}+1 \ldots n_{2}\right) \rightarrow n_{1}+1, \ldots, n_{2}\right] \ldots T\left[\left(n_{m-1}+1 \ldots n\right) \rightarrow n_{m-1}+1, \ldots, n\right] .
\end{aligned}
$$

This formula arises when we take into account all possible attachments of the exchanged gluon between the cascade of gluons and the target gluon. Therefore we need to combine the wave function (initial state) with the fragmentation function (final state radiation) in all possible ways. This is accounted for by performing the summations in (135). The above sum was used to compute the explicit expressions for $\tilde{\Psi}_{2}, \tilde{\Psi}_{3}, \tilde{\Psi}_{4}$ which are given by the Eqs. (125]127[129) by using the formulae for the wave functions and the fragmentation functions. Here we prove that using the Eq. (135) we get the following explicit result for $\tilde{\Psi}_{n}$ (131) in case of the arbitrary $n$ number of gluons

$$
\tilde{\Psi}_{n}(1,2, \ldots n)=g^{n-1} \frac{k_{(1 \ldots n)}}{k_{1} / z_{1}} \frac{1}{\sqrt{z_{1} z_{2} \ldots z_{n}}} \frac{1}{z_{1} z_{2} \ldots z_{n}} \frac{1}{v_{12} v_{23} \ldots v_{n-1 n}} .
$$

As a first step we need to take into account the momentum transfer from the exchanged gluon in the explicit expressions for the wave functions. For example, the wave function $\Psi_{n}$ with $n=m$ (with the momentum transfer included) which appears in the sum (135) has the following form

$$
\begin{aligned}
\Psi_{n}\left(1,2, \ldots, n^{\prime}\right)= & (-1)^{n-1} g^{n-1} \frac{1}{\sqrt{z_{1} z_{2} \ldots z_{n}}} \frac{1}{\xi_{(12 \ldots n-1) n} \xi_{(12 \ldots n-2)(n-1 n) \ldots \xi_{1(2 \ldots n)}}} \\
& \times \frac{1}{v_{(12 \ldots n-1) n^{\prime}} v_{(12 \ldots n-2)(n-1 n)^{\prime} \ldots v_{1(2 \ldots n)^{\prime}}}},
\end{aligned}
$$

where primes indicate the momenta of the wave function before the momentum transfer has happened as discussed in Sec. $[\mathrm{V}$ and shown in Figs. 10 and 11] From the momentum conservation (the transverse momentum of the initial particle is zero) we get following relations between the primed and unprimed momenta

$$
k_{n^{\prime}}=-k_{(1 \ldots n-1)}, k_{(n-1 n)^{\prime}}=-k_{(1 \ldots n-2)}, \ldots, k_{(2 \ldots n)^{\prime}}=-k_{1} \text {. }
$$


Therefore in general we have

$$
v_{(1 \ldots j)(j+1 \ldots n)^{\prime}}=\frac{k_{(1 \ldots j)}}{z_{(1 \ldots j)}}-\frac{k_{(j+1 \ldots n)^{\prime}}}{z_{(j+1 \ldots n)}}=\frac{k_{(1 \ldots j)}}{\xi_{(1 \ldots j)(j+1 \ldots n)}} .
$$

These relations are true for any wave function appearing in expression (135). Using these relations we can rewrite the wave function (137) as

$$
\Psi_{n}\left(1,2, \ldots, n^{\prime}\right)=(-1)^{n-1} g^{n-1} \frac{1}{\sqrt{z_{1} z_{2} \ldots z_{n}}} \frac{1}{k_{1} k_{(12)} \ldots k_{(1 \ldots n-1)}},
$$

which is equivalent to (47). For each term in the sum (135) we have the factor which depends on the longitudinal degrees of freedom in the following way

$$
\begin{aligned}
& \frac{1}{\sqrt{z_{\left(1 \ldots n_{1}\right)} z_{\left(n_{1}+1 \ldots n_{2}\right)} \cdots z_{\left(n_{m-1}+1 \ldots n\right)}}}\left(\frac{z_{\left(1 \ldots n_{1}\right)} z_{\left(n_{1}+1 \ldots n_{2}\right)} \ldots z_{\left(n_{m-1}+1 \ldots n\right)}}{z_{1} \ldots z_{n}}\right)^{3 / 2}= \\
& =\frac{1}{\sqrt{z_{1} \ldots z_{n}}}\left(\frac{z_{\left(1 \ldots n_{1}\right)} z_{\left(n_{1}+1 \ldots n_{2}\right)} \ldots z_{\left(n_{m-1}+1 \ldots n\right)}}{z_{1} \ldots z_{n}}\right) \text {. }
\end{aligned}
$$

Therefore we can factor out the term $1 / \sqrt{z_{1} \ldots z_{n}}$ in front of the whole expression. This factor is purely conventional as it comes from our choice of the normalization of the momenta. The common factor $1 / z_{1} z_{2} \ldots z_{n}$ in the parenthesis () will be left under the sum for the time being. It will prove to be useful to use another notation for the momenta. We introduce auxiliary variables $p_{i i+1}$ defined as follows

$$
p_{12}=k_{1}, p_{23}=k_{(12)}, p_{34}=k_{(123)}, \ldots, p_{n-1 n}=k_{(1 \ldots n-1)} .
$$

Using (135), the new variables, explicit expressions for wave function (138) and fragmentation amplitude we get for the general case $n$

$$
\begin{gathered}
\tilde{\Psi}_{n}(1,2, \ldots, n)=\frac{g^{n-1}}{\sqrt{z_{1} z_{2} \ldots z_{n}}}\left\{\frac{z_{(1 \ldots n)}}{z_{1} \ldots z_{n} v_{12} \ldots v_{n-1 n}}-\right. \\
{\left[\frac{z_{(23 \ldots n)}}{z_{2} \ldots z_{n}} \frac{1}{p_{12} v_{23} \ldots v_{n-1 n}}+\frac{z_{(12)} z_{(3 \ldots n)}}{z_{1} z_{2} \ldots z_{n}} \frac{1}{v_{12} p_{23} v_{34} \ldots v_{n-1 n}}+\ldots+\frac{z_{(1 \ldots n-1)}}{z_{1} z_{2} \ldots z_{n-1}} \frac{1}{v_{12} \ldots v_{n-2 n-1} p_{n-1 n}}\right]+} \\
\sum_{1 \leq<i_{1}<i_{2}<i_{3} \leq n-1} \frac{z_{\left(1 \ldots i_{1}\right)} z_{\left(i_{1}+1 \ldots i_{2}\right)} z_{\left(i_{2}+1 \ldots n\right)}}{z_{1} \ldots z_{n}} \frac{1}{v_{12} \ldots p_{i_{1} i_{1}+1} \ldots p_{i_{2} i_{2}+1} \ldots v_{n-1 n}}- \\
(-1)^{n-1}\left[\frac{z_{(12)}}{z_{1} z_{2}} \frac{1}{v_{12} p_{23} \ldots p_{n-1 n}}+\frac{z_{(23)}}{z_{2} z_{3}} \frac{1}{p_{12} v_{23} p_{34} \ldots p_{n-1 n}}+\ldots+\frac{z_{(n-1 n)}}{z_{n-1} z_{n}} \frac{1}{p_{12} \ldots p_{n-2 n-1} v_{n-1 n}}\right]+ \\
\left.(-1)^{n} \frac{1}{p_{12} p_{23} \ldots p_{n-1 n}}\right\},
\end{gathered}
$$

where $\ldots \ldots \ldots$ means that we need to take higher order sums with $1 \leq i_{1}<i_{2} \cdots<i_{m} \leq n-1$. We see that in the expression above, each term has a form of

$$
\frac{z_{\left(1 \ldots i_{1}\right)} z_{\left(i_{1}+1 \ldots i_{2}\right)} \ldots z_{\left(i_{m}+1 \ldots n\right)}}{z_{1} \ldots z_{n}} \frac{1}{v_{12} \ldots p_{i_{1} i_{1}+1} \ldots p_{i_{2} i_{2}+1} \ldots p_{i_{m} i_{m}+1} \ldots v_{n-1 n}}
$$

with $1 \leq i_{1}<i_{2} \cdots<i_{m} \leq n-1$, where $m$ can range from 0 to $n-1$. In other words we can regard (140) as a sum over the different possible insertions of two kinds of elements $p_{i_{k} i_{k}+1}$ and $v_{j_{l} j_{l}+1}$ into the chain with $n-1$ sites. We write down the above formula in the form with the common denominator, which is equal to

$$
\mathcal{D}_{n}=z_{1} z_{2} \ldots z_{n} v_{12} v_{23} \ldots v_{n-1 n} p_{12} p_{23} \ldots p_{n-1 n} .
$$


The numerator is then equal to (we omitted the $g^{n-1}$ factor here)

$$
\begin{aligned}
& \mathcal{N}_{n}=z_{(1 \ldots n)} p_{12} p_{23} \ldots p_{n-1 n}- \\
& {\left[z_{1} z_{(2 \ldots n)} v_{12} p_{23} \ldots p_{n-1 n}+z_{(12)} z_{(3 \ldots n)} p_{12} v_{23} p_{34} \ldots p_{n-1 n}+\cdots+z_{(1 \ldots n-1)} z_{n} p_{12} \ldots p_{n-2 n-1} v_{n-1 n}\right]+} \\
& \sum_{1 \leq i_{1}<i_{2} \leq n-1} z_{\left(1 \ldots i_{1}\right)} z_{\left(i_{1}+1 \ldots i_{2}\right)} z_{\left(i_{2}+1 \ldots n\right)} p_{12} \ldots v_{i_{1} i_{1}+1} \ldots v_{i_{2} i_{2}+1} \ldots p_{n-1 n}- \\
& \sum_{1 \leq<i_{1}<i_{2}<i_{3} \leq n-1} \frac{z_{\left(1 \ldots i_{1}\right)} z_{\left(i_{1}+1 \ldots i_{2}\right)} z_{\left(i_{2}+1 \ldots i_{3}\right)} z_{\left(i_{3}+1 \ldots n\right)}}{z_{1} \ldots z_{n}} p_{12} \ldots v_{i_{1} i_{1}+1} \ldots v_{i_{2} i_{2}+1} \ldots v_{i_{3} i_{3}+1} \ldots p_{n-1 n} \\
& +\ldots \ldots+ \\
& (-1)^{n-1}\left[z_{(12)} z_{3} \ldots z_{n} p_{12} v_{23} \ldots v_{n-1 n}+z_{1} z_{(23)} \ldots z_{n} v_{12} p_{23} v_{34} \ldots v_{n-1 n}+\ldots+z_{1} \ldots z_{(n-1 n)} v_{12} \ldots v_{n-2 n-1} p_{n-1 n}\right] \\
& +(-1)^{n} z_{1} z_{2} \ldots z_{n} v_{12} \ldots v_{n-1 n} .
\end{aligned}
$$

We assume that the formula (136) is true for $n$. In that case

$$
\mathcal{N}_{n}=z_{1} p_{23} p_{34} \ldots p_{n-1 n} k_{(1 \ldots n)} .
$$

Let us take the case of $n+1$ which reads

$$
\begin{aligned}
& \mathcal{N}_{n+1}=z_{(1 \ldots n+1)} p_{12} p_{23} \ldots p_{n-1 n} p_{n n+1}- \\
& {\left[z_{1} z_{(2 \ldots n+1)} v_{12} p_{23} \ldots p_{n n+1}+z_{(12)} z_{(3 \ldots n+1)} p_{12} v_{23} p_{34} \ldots p_{n n+1}+\cdots+z_{(1 \ldots n)} z_{n+1} p_{12} \ldots p_{n-1 n} v_{n n+1}\right]+} \\
& \sum_{1 \leq i_{1}<i_{2} \leq n} z_{\left(1 \ldots i_{1}\right)} z_{\left(i_{1}+1 \ldots i_{2}\right)} z_{\left(i_{2}+1 \ldots n+1\right)} p_{12} \ldots v_{i_{1} i_{1}+1} \ldots v_{i_{2} i_{2}+1} \ldots p_{n n+1} \\
& \quad+\ldots \ldots+(-1)^{n+1} z_{1} z_{2} \ldots z_{n} z_{n+1} v_{12} \ldots v_{n-1 n} v_{n n+1} .
\end{aligned}
$$

Obviously in every term in $\mathcal{N}_{n+1}$ there can be either $p_{n n+1}$ or $v_{n n+1}$ present. We collect these terms and obtain

$$
\begin{array}{r}
\mathcal{N}_{n+1}=p_{n n+1}(\ldots \ldots)+z_{n+1} v_{n n+1}\left[-z_{(1 \ldots n)} p_{12} \ldots p_{n-1 n}+\right. \\
z_{1} z_{(2 \ldots n)} v_{12} p_{23} \ldots p_{n-1 n}+z_{(12)} z_{(3 \ldots n)} p_{12} v_{23} p_{34} \ldots p_{n-1 n}+\cdots+z_{(1 \ldots n-1)} z_{n} p_{12} \ldots p_{n-2 n-1} v_{n-1 n} \\
-\sum_{1 \leq i_{1}<i_{2} \leq n-1} z_{\left(1 \ldots i_{1}\right)} z_{\left(i_{1}+1 \ldots i_{2}\right)} z_{\left(i_{2}+1 \ldots n\right)} p_{12} \ldots v_{i_{1} i_{1}+1} \ldots v_{i_{2} i_{2}+1} \ldots p_{n-1 n}+ \\
\left.\quad+\ldots \ldots+(-1)^{n+1} z_{1} z_{2} \ldots z_{n} v_{12} \ldots v_{n-1 n}\right] .
\end{array}
$$

The terms in the squared parenthesis [...] combine to $-\mathcal{N}_{n}$ of (142). We make the inductive step and use (143) for this expression. Using $p_{n n+1}=k_{(1 \ldots n)}$ the numerator $\mathcal{N}_{n+1}$ reads then

$$
\begin{aligned}
& \mathcal{N}_{n+1}=p_{n n+1}\left\{z_{(1 \ldots n+1)} p_{12} p_{23} \ldots p_{n-1 n}-\right. \\
& {\left[z_{1} z_{(2 \ldots n+1)} v_{12} p_{23} \ldots p_{n-1 n}+z_{(12)} z_{(3 \ldots n+1)} p_{12} v_{23} p_{34} \ldots p_{n-1 n}+\cdots+z_{(1 \ldots n-1)} z_{(n n+1)} p_{12} \ldots p_{n-2 n-1} v_{n-1 n}\right]+} \\
& \sum_{1 \leq i_{1}<i_{2} \leq n-1} z_{\left(1 \ldots i_{1}\right)} z_{\left(i_{1}+1 \ldots i_{2}\right)} z_{\left(i_{2}+1 \ldots n+1\right)} p_{12} \ldots v_{i_{1} i_{1}+1} \ldots v_{i_{2} i_{2}+1} \ldots p_{n-1 n}- \\
& \ldots \ldots \ldots \\
& \ldots
\end{aligned}
$$

where the last term comes from making the inductive step as described above and we have factored out $p_{n n+1}$. Now we combine terms in the parenthesis $\{\ldots\}$ which contain either $p_{n-1 n}$ or $v_{n-1 n}$. We then observe that the coefficient in front of $z_{(n n+1)} v_{n-1 n}$ will combine to $\mathcal{N}_{n-1}$ and we can again use (143) for $n-1$. Therefore the term proportional to $v_{n-1 n}$ in $\{\ldots\}$ reads

$$
-z_{(n n+1)} v_{n-1 n} z_{1} p_{23} p_{34} \ldots p_{n-1 n} .
$$

Therefore all the terms now contain $p_{n-1 n}$ and it can be again factored out. We see that we need to apply this procedure recursively $n-1$ times, and each time we pick up a term which will be proportional to $z_{(j \ldots n+1)} v_{j j+1}$ 
(where $j$ decreases with each step). Making the inductive step we see that such term will contain $p_{j j+1}$ which can be factored out. After performing all these steps the result reads then

$$
\mathcal{N}_{n+1}=p_{23} \ldots p_{n-1 n} p_{n n+1}\left[z_{(1 \ldots n+1)} p_{12}-z_{1} z_{(2 \ldots n+1)} v_{12}-z_{1} z_{(3 \ldots n+1)} v_{23}-\cdots-z_{1} z_{(n n+1)} v_{n-1 n}-z_{1} z_{n+1} v_{n n+1}\right],
$$

where all $p_{j j+1}$ terms with $2 \leq j \leq n$ have been factored out. We can use

$$
-z_{1} z_{(k \ldots n+1)} v_{1 k}-z_{1} z_{(k+1 \ldots n+1)} v_{k k+1}=-z_{1} z_{k} v_{1 k}-z_{1} z_{(k+1 \ldots n+1)}\left(v_{1 k}+v_{k k+1}\right)=-z_{1} z_{k} v_{1 k}-z_{1} z_{(k+1 \ldots n+1)} v_{1 k+1},
$$

to rewrite the numerator as

$$
\mathcal{N}_{n+1}=p_{23} \ldots p_{n-1 n} p_{n n+1}\left[z_{(1 \ldots n+1)} p_{12}-z_{1} z_{2} v_{12}-z_{1} z_{3} v_{13}-\cdots-z_{1} z_{n} v_{1 n}-z_{1} z_{n+1} v_{1 n+1}\right] .
$$

Using $p_{12}=k_{1}$ and $z_{1} z_{j} v_{1 j}=z_{j} k_{1}-z_{1} k_{j}$ we find that the terms in the [...] combine to $z_{1} k_{(1 \ldots n+1)}$ which gives

$$
\mathcal{N}_{n+1}=z_{1} p_{23} \ldots p_{n-1 n} p_{n n+1} k_{(1 \ldots n+1)},
$$

and this completes the proof for $\tilde{\Psi}_{n+1}$. Let us finally note that in the relations (147) to (150) were also used in the explicit calculations done for $n=2,3,4$. It is important to note that these relations hold also in the cases where $z_{(1 \ldots n+1)} \neq 0$, which is important as in the proof we recursively move to lower $n$ where the partial sums over longitudinal $z$ and transverse momenta $k$ can take different values.

[1] A. H. Mueller, Nucl. Phys. B 415, 373 (1994).

[2] Z. Chen and A. H. Mueller, Nucl. Phys. B 451, 579 (1995).

[3] S. Weinberg, Phys. Rev. 150, 1313 (1966).

[4] J. D. Bjorken, J. B. Kogut and D. E. Soper, Phys. Rev. D 3, 1382 (1971).

[5] G. P. Lepage and S. J. Brodsky, Phys. Rev. D 22, 2157 (1980).

[6] S. J. Brodsky, H. C. Pauli and S. S. Pinsky, Phys. Rept. 301, 299 (1998) arXiv:hep-ph/9705477.

[7] E.A. Kuraev, L.N. Lipatov and V.S. Fadin, Sh. Eksp. Teor. Fiz. 72 (1977) 373, ( Sov. Phys. JETP 45 (1977) 199); Ya. Ya. Balitzkij and L.N. Lipatov, Yad. Fiz. 28 (1978) 1597 ( Sov. J. Nucl. Phys. 28 (1978) 822), J.B. Bronzan and R.L. Sugar, Phys. Rev. D17 (1978) 585; T. Jaroszewicz, Acta. Phys. Polon. B11 (1980) 965.

[8] S. J. Parke and T. R. Taylor, Phys. Rev. Lett. 56, 2459 (1986).

[9] M. L. Mangano and S. J. Parke, Phys. Rept. 200 (1991) 301 arXiv:hep-th/0509223.

[10] I. Balitsky and G. A. Chirilli, Phys. Rev. D 77, 014019 (2008) arXiv:0710.4330 [hep-ph]].

[11] V. S. Fadin and R. Fiore, Phys. Lett. B 661, 139 (2008) arXiv:0712.3901 [hep-ph]].

[12] V. S. Fadin, R. Fiore, A. V. Grabovsky and A. Papa, Nucl. Phys. B 784, 49 (2007) arXiv:0705.1885 [hep-ph]].

[13] V. S. Fadin, R. Fiore and A. Papa, Phys. Lett. B 647, 179 (2007) arXiv:hep-ph/0701075.

[14] V. S. Fadin, R. Fiore and A. Papa, Nucl. Phys. B 769, 108 (2007) arXiv:hep-ph/0612284.

[15] G. P. Salam, Nucl. Phys. B 461, 512 (1996) arXiv:hep-ph/9509353.

[16] A. H. Mueller and G. P. Salam, Nucl. Phys. B 475, 293 (1996) arXiv:hep-ph/9605302].

[17] Yu. V. Kovchegov, Phys. Rev. D 60, 034008 (1999) arXiv:hep-ph/9901281.

[18] Yu. V. Kovchegov, Phys. Rev. D 61 (2000) 074018 arXiv:hep-ph/9905214.

[19] I. Balitsky, Nucl. Phys. B 463, 99 (1996) arXiv:hep-ph/9509348.

[20] I. Balitsky, Phys. Rev. Lett. 81, 2024 (1998) arXiv:hep-ph/9807434];

Phys. Rev. D 60, 014020 (1999) arXiv:hep-ph/9812311.

[21] I. Balitsky, Phys. Lett. B 518, 235 (2001) arXiv:hep-ph/0105334].

[22] I. Balitsky and A. V. Belitsky, Nucl. Phys. B 629, 290 (2002) arXiv:hep-ph/0110158.

[23] I. Balitsky, Phys. Rev. D 70, 114030 (2004) arXiv:hep-ph/0409314.

[24] I. Balitsky, Nucl. Phys. Proc. Suppl. 152, 275 (2006).

[25] I. Balitsky, Phys. Rev. D 72, 074027 (2005) arXiv:hep-ph/0507237.

[26] L. McLerran and R. Venugopalan, Phys. Rev. D49 (1994) 2233; Phys. Rev. D49 (1994) 3352;

Phys. Rev. D50 (1994) 2225; R. Venugopalan, Acta Phys. Polon. B30 (199) 3731;

E. Iancu, A. Leonidov and L. McLerran, Nucl.Phys. A692 (2001) 583;

E. Ferreiro, E. Iancu, A. Leonidov and L. McLerran, hep-ph/0109115.

[27] E. Iancu and L. McLerran, Phys. Lett. B510 (2001) 133.

[28] J. Jalilian-Marian, A. Kovner, L. McLerran and H. Weigert, Nucl. Phys. B504 (1997) 415; Phys. Rev. D59 (1999) 014014; Phys. Rev. D59 (1999) 034007.

[29] E. Iancu and R. Venugopalan, arXiv:hep-ph/0303204

[30] V. S. Fadin and L. N. Lipatov, Nucl. Phys. B 477, 767 (1996) arXiv:hep-ph/9602287. 
[31] V. S. Fadin and L. N. Lipatov, Phys. Lett. B 429, 127 (1998) arXiv:hep-ph/9802290].

[32] G. Camici and M. Ciafaloni, Phys. Lett. B 412, 396 (1997) [Erratum-ibid. B 417, 390 (1998)] arXiv:hep-ph/9707390.

[33] M. Ciafaloni and G. Camici, Phys. Lett. B 430, 349 (1998) arXiv:hep-ph/9803389].

[34] V. S. Fadin and R. Fiore, Phys. Rev. D 72, 014018 (2005) arXiv:hep-ph/0502045.

[35] V. S. Fadin and R. Fiore, Phys. Lett. B 610, 61 (2005) [Erratum-ibid. B 621, 61 (2005)] arXiv:hep-ph/0412386].

[36] I. Balitsky, Phys. Rev. D 75, 014001 (2007) arXiv:hep-ph/0609105.

[37] Yu. V. Kovchegov and H. Weigert, Nucl. Phys. A 784, 188 (2007) arXiv:hep-ph/0609090.

[38] B. Andersson, G. Gustafson, H. Kharraziha and J. Samuelsson, Z. Phys. C 71, 613 (1996).

[39] B. Andersson, G. Gustafson and J. Samuelsson, Nucl. Phys. B 467, 443 (1996).

[40] J. Kwiecinski, A. D. Martin and P. J. Sutton, Z. Phys. C 71, 585 (1996) arXiv:hep-ph/9602320.

[41] G. P. Salam, JHEP 9807, 019 (1998) arXiv:hep-ph/9806482.

[42] D. A. Ross, Phys. Lett. B 431, 161 (1998) arXiv:hep-ph/9804332.

[43] J. Kwiecinski, A. D. Martin and A. M. Stasto, Phys. Rev. D 56, 3991 (1997) arXiv:hep-ph/9703445.

[44] E. Avsar, G. Gustafson and L. Lonnblad, JHEP 0507, 062 (2005) arXiv:hep-ph/0503181.

[45] E. Avsar, G. Gustafson and L. Lonnblad, JHEP 0701, 012 (2007) arXiv:hep-ph/0610157.

[46] G. P. Salam, Acta Phys. Polon. B 30, 3679 (1999) arXiv:hep-ph/9910492.

[47] M. Ciafaloni and D. Colferai, Phys. Lett. B 452, 372 (1999) arXiv:hep-ph/9812366].

[48] M. Ciafaloni, D. Colferai and G. P. Salam, Phys. Rev. D 60, 114036 (1999) arXiv:hep-ph/9905566.

[49] M. Ciafaloni, D. Colferai and G. P. Salam, JHEP 9910, 017 (1999) arXiv:hep-ph/9907409.

[50] M. Ciafaloni, D. Colferai, G. P. Salam and A. M. Stasto, Phys. Lett. B 576, 143 (2003) arXiv:hep-ph/0305254].

[51] M. Ciafaloni, D. Colferai, G. P. Salam and A. M. Stasto, Phys. Rev. D 68, 114003 (2003) arXiv:hep-ph/0307188.

[52] M. Ciafaloni, D. Colferai, G. P. Salam and A. M. Stasto, JHEP 0708, 046 (2007) arXiv:0707.1453 [hep-ph]].

[53] G. Altarelli, R. D. Ball and S. Forte, Nucl. Phys. B 575, (2000) 313;

[54] G. Altarelli, R. D. Ball and S. Forte, Nucl. Phys. B 599, (2001) 383.

[55] G. Altarelli, R. D. Ball and S. Forte, arXiv:hep-ph/0104246.

[56] G. Altarelli, R. D. Ball and S. Forte, Nucl. Phys. B 621, (2002) 359;

[57] G. Altarelli, R. D. Ball and S. Forte, Nucl. Phys. B 674, (2003) 459;

[58] G. Altarelli, R. D. Ball and S. Forte, Nucl. Phys. B 742, (2006) 1.

[59] R.S. Thorne, Phys. Rev. D64 (2001) 074005; Phys. Lett. 474 (2000) 372.

[60] C. D. White and R. S. Thorne, Phys. Rev. D 75, (2007) 034005.

[61] A. V. Manohar, arXiv:hep-ph/9802419

[62] I.S. Gradshteyn and I.M. Ryzhik, Table of Integrals, Series and Products, VIth edition, Academic Press, 2000.

[63] Yu. L. Dokshitzer, G. Marchesini and G. P. Salam, Phys. Lett. B 634, 504 (2006) arXiv:hep-ph/0511302.

[64] Yu. L. Dokshitzer and G. Marchesini, Phys. Lett. B 646, 189 (2007) arXiv:hep-th/0612248.

[65] G. Marchesini, Talk given at Workshop on Future Prospects in QCD at High Energies, Brookhaven, Upton, New York, 17-22 Jul 2006; arXiv:hep-ph/0605262.

[66] J. Bartels, Phys. Lett. B 298, 204 (1993).

[67] J. Bartels, Z. Phys. C 60, 471 (1993).

[68] K. J. Golec-Biernat and A. M. Stasto, Nucl. Phys. B 668, 345 (2003) arXiv:hep-ph/0306279.

[69] M. Froissart, Phys. Rev. 123 (1961) 1053; A. Martin, Phys. Rev. 129 (1963) 1432.

[70] A. Kovner and U. A. Wiedemann, Phys. Rev. D 66, 034031 (2002) arXiv:hep-ph/0204277.

[71] A. Kovner and U. A. Wiedemann, Phys. Rev. D 66, 051502 (2002) arXiv:hep-ph/0112140.

[72] A. Kovner and U. A. Wiedemann, Phys. Lett. B 551, 311 (2003) arXiv:hep-ph/0207335.

[73] W. Heisenberg, Z. Phys. 133, (1952) 65.

[74] V. Del Duca, Phys. Rev. D 52, 1527 (1995) arXiv:hep-ph/9503340.

[75] V. Del Duca, Phys. Rev. D 48, 5133 (1993) arXiv:hep-ph/9304259. 